\title{
ASYMPTOTICALLY HOLOMORPHIC FUNCTIONS AND TRANSLATION INVARIANT SUBSPACES OF WEIGHTED HILBERT SPACES OF SEQUENCES
}

\author{
By JEAN ESTERLE AND ALEXANDER VOLBERG ${ }^{1}$
}

ABSTRACT. - Let $\omega$ be a weight on $\mathbb{Z}$ and assume that the spectrum of the usual shift operator on the weighted space $\ell_{\omega}^{2}(\mathbb{Z})$ equals the unit circle. We show that if $\sum_{n<0} \frac{\log \omega(n)}{n^{2}}=+\infty$, and if the sequence $(\omega(-n))_{n \geqslant 0}$ satisfies suitable growth and regularity conditions, then all nontrivial translation invariant subspaces of $\ell_{\omega}^{2}(\mathbb{Z})$ are generated by their intersection with

$$
\ell_{\omega}^{2}\left(\mathbb{Z}^{+}\right)=\left\{u=\left(u_{n}\right)_{n \in \mathbb{Z}} \in \ell_{\omega}^{2}(\mathbb{Z}) \mid u_{n}=0(n<0)\right\} .
$$

When $\omega(n)=1$ for $n \geqslant 0$ and $\omega(n)=\mathrm{e}^{|n| / \log (|n|+1)}$ for $n<0$, this shows that every nontrivial translation invariant subspace of $\ell_{\omega}^{2}(\mathbb{Z})$ is generated by the translates of the Fourier sequence of some singular inner function.

The proofs are based on a priori estimates on the growth of the solutions of some convolution equations, obtained by using the theory of asymptotically holomorphic functions in the disc.

(C) 2002 Éditions scientifiques et médicales Elsevier SAS

RÉSUMÉ. - Soit $\omega$ un poids sur $\mathbb{Z}$ tel que le spectre de l'opérateur de décalage sur l'espace pondéré $\ell_{\omega}^{2}(\mathbb{Z})$ soit égal au cercle unité. On montre que si $\sum_{n<0} \frac{\log \omega(n)}{n^{2}}=+\infty$, et si la suite $(\omega(-n))_{n \geqslant 0}$ satisfait des conditions de croissance et de régularité convenables, alors tous les sous-espaces invariants par translation non triviaux de $\ell_{\omega}^{2}(\mathbb{Z})$ sont engendrés par leur intersection avec

$$
\ell_{\omega}^{2}\left(\mathbb{Z}^{+}\right)=\left\{u=\left(u_{n}\right)_{n \in \mathbb{Z}} \in \ell_{\omega}^{2}(\mathbb{Z}) \mid u_{n}=0(n<0)\right\}
$$

Quand $\omega(n)=1$ pour $n \geqslant 0$ et $\omega(n)=\mathrm{e}^{|n| / \log (|n|+1)}$ pour $n<0$, ceci montre que tout sous-espace invariant par translation non trivial de $\ell_{\omega}^{2}(\mathbb{Z})$ est engendré par les translatés de la suite des coefficients de Fourier d'une fonction intérieure singulière.

Les démonstrations sont basées sur des estimations a priori de la croissance des solutions de certaines équations de convolution, obtenues en utilisant la théorie des fonctions asymptotiquement holomorphes dans le disque.

(C) 2002 Éditions scientifiques et médicales Elsevier SAS

\footnotetext{
${ }^{1}$ The work of the first named author is part of the research program of the European network 'Analysis and operators', contract HPRN-CT-00116-2000, supported by the European Commission. The second named author was supported by NSF Grant DMS 9622936 and by Grant BSF 00030 from the United States-Israel Binational Science Foundation.
} 


\section{Introduction}

Let $\omega$ be a weight on $\mathbb{Z}$, and assume that the usual shift operator $S:\left(u_{n}\right)_{n \in \mathbb{Z}} \rightarrow\left(u_{n-1}\right)_{n \in \mathbb{Z}}$ is well-defined, bounded and invertible on

$$
\ell_{\omega}^{2}(\mathbb{Z})=\left\{u=\left.\left(u_{n}\right)_{n \in \mathbb{Z}}\left|\sum_{n \in \mathbb{Z}}\right| u_{n}\right|^{2} \omega^{2}(n)<+\infty\right\}
$$

(we refer to [44] and [48] for general properties of the shift operator). A closed subspace $M$ of $\ell_{\omega}^{2}(\mathbb{Z})$ is said to be translation-invariant (resp. left-invariant, resp. right-invariant) when $S(M)=M$ (resp. $S^{-1}(M) \subset M$, resp. $S(M) \subset M$ ).

In this paper, we are interested in the case where the spectrum $\sigma(S)$ of $S$ equals the unit circle $\mathbb{T}$. In this situation, the Laurent expansions $\tilde{u}(z)=\sum_{n \in \mathbb{Z}} u_{n} z^{n}$ "live" on the unit circle for $u=\left(u_{n}\right)_{n \in \mathbb{Z}} \in \ell_{\omega}^{2}(\mathbb{Z})$ (these formal Laurent series expansions can be interpreted as hyperfunctions on $\mathbb{T}$, see Section 3 ), and the Taylor expansions $\tilde{u}(z)=\sum_{n=0}^{\infty} u_{n} z^{n}$ are analytic on the open unit disc $\mathbb{D}$ for

$$
u=\left(u_{n}\right)_{n \geqslant 0} \in \ell_{\omega}^{2}\left(\mathbb{Z}^{+}\right)=\left\{\left.\left(u_{n}\right)_{n \geqslant 0}\left|\sum_{n=0}^{\infty}\right| u_{n}\right|^{2} \omega^{2}(n)<+\infty\right\} .
$$

Notice that in both cases, we have $\widetilde{S u}(z)=z \tilde{u}(z)$.

We can identify $\ell_{\omega}^{2}\left(\mathbb{Z}^{+}\right)$with the space $\left\{u=\left(u_{n}\right)_{n \in \mathbb{Z}} \in \ell_{\omega}^{2}(\mathbb{Z}) \mid u_{n}=0(n<0)\right\}$. Clearly, if $M$ is a translation invariant subspace of $\ell_{\omega}^{2}(\mathbb{Z})$, then $M_{+}=M \cap \ell_{\omega}^{2}\left(\mathbb{Z}^{+}\right)$has the "division property": if $u \in M_{+}$and if $\tilde{u}\left(z_{0}\right)=0$, where $z_{0} \in \mathbb{D}$, then the function $\frac{\tilde{u}(z)}{z-z_{0}}$ is in $\widetilde{M}_{+}$(or, equivalently, $\left.\left(S-z_{0} I\right)^{-1} u \in M_{+}\right)$. Now let $N$ be a right-invariant subspace of $\ell_{\omega}^{2}\left(\mathbb{Z}^{+}\right)$. It is not difficult to check, and well-known, that $N$ has the division property if and only if $\sigma\left(S_{N}\right) \subset \mathbb{T}$ where $S_{N}: f+N \rightarrow S f+N$ is the map induced by $S$ on the quotient space $\ell_{\omega}^{2}\left(\mathbb{Z}^{+}\right) / N$.

The issue at hand is the following

Problem 1. - Given a right-invariant subspace $N$ of $\ell_{\omega}^{2}\left(\mathbb{Z}^{+}\right)$having the division property, does there exist a translation invariant subspace $M$ of $\ell_{\omega}^{2}\left(\mathbb{Z}^{+}\right)$such that $M \cap \ell_{\omega}^{2}\left(\mathbb{Z}^{+}\right)=N$ ?

Problem 2. - Given a translation invariant subspace $M$ of $\ell_{\omega}^{2}(\mathbb{Z})$, is it generated by its intersection with $\ell_{\omega}^{2}\left(\mathbb{Z}^{+}\right)$(which constitutes a right-invariant subspace of $\ell_{\omega}^{2}\left(\mathbb{Z}^{+}\right)$having the division property)?

It follows from Wiener's characterization of translation invariant subspaces of $\ell^{2}(\mathbb{Z})$ [51] that $\bigvee_{n \in \mathbb{Z}} S^{n} u=\ell^{2}(\mathbb{Z})$ for every $u \in \ell^{2}\left(\mathbb{Z}^{+}\right) \backslash\{0\}$, and so the answer to both problems is of course negative in general.

On the other hand, it was shown in [25, Theorem 3.7] that the answer to Problem 1 is positive if $\omega(n)=1$ for $n \geqslant 0$ and if $\varliminf_{n \rightarrow \infty} \frac{\log \omega(-n)}{\sqrt{ } n}=+\infty$. A general discussion of Problem 1 is given by the authors in [31]: there exists a sequence $\left(\omega_{+}^{(p)}\right)_{p \geqslant 1}$ of weights on $\mathbb{Z}^{+}$, which depends only on $\omega_{+}:=\left.\omega\right|_{\mathbb{Z}^{+}}$such that the answer to Problem 1 is positive whenever $\sum_{n \geqslant 0} \frac{\omega_{+}^{(p)}(n)^{2}}{\omega(-n)^{2}}=+\infty$ for every $p \geqslant 1$, and $\omega_{+}^{(p)}(n)^{1 / n} \rightarrow_{n \rightarrow \infty} 1$ for every $p \geqslant 1$ (the precise definition of these weights $\omega_{+}^{(p)}$ is given in Section 5). The proof of this result relies on elementary operator theoretical arguments, but the estimation of the weights $\omega_{+}^{(p)}$ in concrete cases relies on sharp estimates of the growth of a quotient of two analytic functions in the disc [42]. 
Before discussing Problem 2, which is much harder, it seems worth mentioning that analogous problems have been studied for a large class of Banach spaces of functions analytic on a multiply connected domain $\Omega$ by Abkar and Hedenmalm [1] (see [35] for a previous discussion of these problems for Banach algebras).

Assume, for example, that $\Omega$ is the annulus $\{z \in \mathbb{C}|\rho<| z \mid<1\}$, and set $\Omega_{1}=\mathbb{D}$, $\Omega_{2}=\mathbb{C} \backslash \rho \overline{\mathbb{D}}$, and let $B$ be a Banach space of functions analytic on $\Omega$ which contains all rational functions with poles in $\mathbb{C}_{\infty} \backslash \bar{\Omega}$. Assume also that evaluation at $\lambda$ is continuous on $B$ for $\lambda \in \Omega$ and that the function $z \rightarrow \frac{f(z)}{z-\lambda}$ belongs to $B$ if $f \in B, \lambda \in \Omega, f(\lambda)=0$.

Denote by $\mathcal{M}(B)$ the algebra of multipliers on $B$, i.e. the algebra of functions $\varphi$ analytic on $\Omega$ such that $\varphi B \subset B$. A closed subspace $J$ of $B$ is said to be $\mathcal{M}(B)$-invariant if $\varphi J \subset J$ for every $J \in B$. Also, denote by $B_{1}$ (resp. $B_{2}$ ) the closed subspace of $B$ consisting of all $f \in B$ which can be analytically extended to $\Omega_{1}$ (resp. $\left.\Omega_{2}\right)$ and define $\mathcal{M}\left(B_{1}\right)$ and $\mathcal{M}\left(B_{1}\right)$-invariant subspaces of $B_{1}$ as above.

For $f \in B$ set $M_{z}(f)(\xi)=\xi f(\xi) \quad(\xi \in \Omega)$ and if $J$ is $\mathcal{M}(B)$-invariant denote by $M_{z, J}: B / J \rightarrow B / J$ the map $f+J \rightarrow M_{z}(f)+J$. Define in the same way $M_{z, I}$ if $I$ is a $\mathcal{M}\left(B_{1}\right)$-invariant subspace of $B_{1}$.

Now, denote by $\mathcal{U}$ the lattice of $\mathcal{M}(B)$-invariant subspaces $J$ of $B$ such that $\sigma\left(M_{z, J}\right) \subset \Omega_{2}$ and denote by $\mathcal{U}^{+}$the lattice of $\mathcal{M}\left(B_{1}\right)$-invariant subspaces $I$ of $B_{1}$ such that $\sigma\left(M_{z, I}\right) \subset \Omega_{2}$. For $A \subset B$ denote by $\mathcal{M}(B)$. $A$ the set of all elements of the form $R a, R \in \mathcal{M}(B), a \in A$. It is shown in [1] that if $B$ satisfies suitable regularity conditions with respect to $\Omega_{1}$ and $\Omega_{2}$, then $I=[M(B) \cdot I]^{-} \cap B_{1}$ for every $I \in \mathcal{U}^{+}$and $J=\left[M(B) \cdot\left(J \cap B_{1}\right)\right]^{-}$for every $J \in \mathcal{U}$. In other terms, in this context the natural version of Problem 1 has a positive answer for $I \in \mathcal{U}^{+}$, the natural version of Problem 2 has a positive answer for $J \in \mathcal{U}$, and the map $J \rightarrow J \cap B_{1}$ is then a bijection from $\mathcal{U}$ onto $\mathcal{U}^{+}$. These results are based on a factorization theorem [1, Lemma 3.7]: if $f \in B$ then there exists $f_{1} \in B_{1}$ and $f_{2} \in B_{2}$ such that $f=f_{1} \cdot f_{2}$, with some control on the zero sets of $f_{1}$ and $f_{2}$.

We now go back to Problem 2 for $\ell_{\omega}^{2}(\mathbb{Z})$, where $\omega$ is a weight for which the spectrum of the shift operator $S$ equals the unit circle. The commutant $\mathcal{M}_{\omega}$ of $S$ can be as well known identified to the space of elements $h=\left(h_{n}\right)_{n \in \mathbb{Z}}$ of $\ell_{\omega}^{2}(\mathbb{Z})$ such that $h * u \in \ell_{\omega}^{2}(\mathbb{Z})$ for every $u \in \ell_{\omega}^{2}(\mathbb{Z})$, where $(h * u)_{n}$ is the limit in the sense of Cesaro of the sequence $\left(\sum_{|m| \leqslant p} h_{m} u_{n-m}\right)_{p \geqslant 0}$, see Section 5, and the translation invariant subspaces of $\ell_{\omega}^{2}(\mathbb{Z})$ are exactly the $\mathcal{M}_{\omega}$-invariant subspaces. The open set $\Omega_{1}$ of the above discussion is now the unit disc, $B_{1}$ becomes $\ell_{\omega}^{2}\left(\mathbb{Z}^{+}\right)$and the $\mathcal{M}\left(B_{1}\right)$-invariant subspaces of $B_{1}$ become the right-invariant subspaces of $\ell_{\omega}^{2}\left(\mathbb{Z}^{+}\right)$. Unfortunately, the open set $\Omega_{2}=\mathbb{C} \backslash \rho \overline{\mathbb{D}}$ becomes $\mathbb{C} \backslash \overline{\mathbb{D}}$, and $\sigma\left(S_{M}\right) \subset \mathbb{T}$, so that $\sigma\left(S_{M}\right) \cap(\mathbb{C} \backslash \overline{\mathbb{D}})=\phi$ for every nontrivial translation invariant subspace $M$ of $\ell_{\omega}^{2}(\mathbb{Z})$. We thus see that Problem 2 in this context is not a limit case of the results obtained in [1] for spaces of holomorphic functions.

Let $\left(\omega_{+}^{(p)}\right)_{p \geqslant 1}$ be the sequence of weights on $\mathbb{Z}^{+}$mentioned above, and assume that $(\omega(-n))_{n \geqslant 0}$ satisfies the growth condition

$$
\sum_{n=0}^{\infty} \frac{\omega_{+}^{(p)}(n)^{2}}{\omega(-n)^{2}}=+\infty \quad(p \geqslant 1) .
$$

In this situation we know from [31] that the answer to Problem 1 is positive, and in fact we know more precisely that $N=\left[\bigvee_{n \leqslant 0} S^{n}(N)\right] \cap \ell_{\omega}^{2}\left(\mathbb{Z}^{+}\right)$and $\left[\bigvee_{n \leqslant 0} S^{n}(N)\right]+\ell_{\omega}^{2}\left(\mathbb{Z}^{+}\right)=\ell_{\omega}^{2}(\mathbb{Z})$ for every right-invariant subspace $N$ of $\ell_{\omega}^{2}\left(\mathbb{Z}^{+}\right)$having the division property. It is then easy to see that if $M$ is a translation invariant subspace of $\ell_{\omega}^{2}(\mathbb{Z})$, then $M=\bigvee_{n \leqslant 0} S^{n}\left(M^{+}\right)$if $M^{+}:=M \cap \ell_{\omega}^{2}\left(\mathbb{Z}^{+}\right)$does not reduce to $\{0\}$. So, for weights satisfying $(*)$, Problem 2 will 
have a positive answer if $M \cap \ell_{\omega}^{2}\left(\mathbb{Z}^{+}\right) \neq\{0\}$ for every nonzero translation invariant subspace of $\ell_{\omega}^{2}(\mathbb{Z})$.

The starting point of our strategy is a factorization theorem due to Borichev [14]. Set $\bar{\omega}_{+}(n)=\sup _{p \geqslant 0} \frac{\omega(n)}{\omega(n+p)}$ for $n \geqslant 0$, and for $s>0$ set $\omega_{(s)}(n)=\omega(n)$ for $n \geqslant 0, \omega_{(s)}(n)=\omega^{s}(n)$ for $n<0$. Using essentially the same method as in [14] and [17], we give in Theorem B.5 of Appendix B a quantitative version of [18, Theorem 6.1]. Assume that $\omega$ satisfies the six following conditions:

(1) $\sigma(S) \subset \mathbb{T}$

(2) $\sum_{n<0} \frac{\log \omega(n)}{n^{2}}=+\infty$;

(3) $\left(\frac{\log \omega(-n)}{n}(\log n)^{A}\right)_{n \geqslant 1}$ is eventually increasing for some $A>0$;

(4) $(\omega(-n))_{n \geqslant 1}$ is eventually log-concave;

(5) $\limsup _{n \rightarrow \infty} \frac{\log \bar{\omega}_{+}(n)}{\log \omega(-n)}<1 / 4$;

(6) $\limsup _{n \rightarrow \infty} \frac{\log ^{+} \omega^{-1}(n)}{\log \omega(-n)}<1 / 64$.

Then, for every $u \in \ell_{\omega}^{2}(\mathbb{Z})$ and every $s<1 / 4$, there exist $w \in \ell_{\omega}^{2}\left(\mathbb{Z}^{+}\right), k \geqslant 0$ and $h \in \mathcal{M}_{\omega_{(s)}}$, with $h_{n}=0$ for $n \geqslant 0$, such that $w=\mathrm{e}^{-h} * S^{k} u$, where $\mathrm{e}^{h}$ is computed with respect to convolution in $\mathcal{M}_{\omega_{(s)}}$.

Conditions (3), (4) and (5) imply that $\omega$ satisfies (*), and if it were possible to arrange that $h \in \mathcal{M}_{\omega}$ in this factorization, then we would have $\bigvee_{n \in \mathbb{Z}} S^{n} u=\bigvee_{n \in \mathbb{Z}} S^{n-k} w$ for every $u \in \ell_{\omega}^{2}(\mathbb{Z})$, and so the answer to Problem 2 would be positive. But, as observed by the first author in [27], there are no weights on $\mathbb{Z}$ for which such a nice factorization in $\ell_{\omega}^{2}(\mathbb{Z})$ is available.

Now, introduce the following conditions:

(4') $\left(\omega(-n) / n^{\alpha}\right)_{n \geqslant 1}$ is eventually log-concave for some $\alpha>3 / 2$;

(5') $\limsup _{n \rightarrow \infty} \frac{\log \bar{\omega}_{+}(n)}{\log \omega(-n)}<1 / 200$.

The main result of the paper is Theorem 5.8, which shows that if $\omega$ satisfies (1), (2), (3), (4') and (5'), we have $\bigvee_{n \leqslant 0} S^{n} u=\bigvee_{n \leqslant-k} S^{n} w$ in the factorization above, despite the fact that in general $h \notin \mathcal{M}_{\omega}$. So for these weights both problems (1) and (2) have a positive answer, and the map $M \rightarrow M \cap \ell_{\omega}^{2}\left(\mathbb{Z}^{+}\right)$is a bijection from the lattice of translation invariant subspaces of $\ell_{\omega}^{2}(\mathbb{Z})$ onto the lattice of right-invariant subspaces of $\ell_{\omega}^{2}\left(\mathbb{Z}^{+}\right)$having the division property (and the inverse map is the map $N \rightarrow \bigvee_{n \leqslant 0} S^{n}(N)$ ). More generally, every left-invariant subspace of $\ell_{\omega}^{2}(\mathbb{Z})$ has the form $M=\bigvee_{n \leqslant-k} S^{n}(N)$, where $k \geqslant 0$, and where $N$ is a closed subspace of $\ell_{\omega}^{2}\left(\mathbb{Z}^{+}\right)$having the division property.

We now outline the strategy of the proof of Theorem 5.8, which is based on the theory of almost analytic functions in the disc developed in [17,50]. Set $\omega^{*}(n)=\omega(-n-1)^{-1}$ for $n \in \mathbb{Z}$. Using the formula $\langle u, v\rangle=\sum_{n \in \mathbb{Z}} u_{n} v_{-n-1}$ for $u=\left(u_{n}\right)_{n \in \mathbb{Z}} \in \ell_{\omega}^{2}(\mathbb{Z}), v=\left(v_{n}\right)_{n \in \mathbb{Z}} \in \ell_{\omega^{*}}^{2}(\mathbb{Z})$, we can identify the dual of $\ell_{\omega}^{2}(\mathbb{Z})$ with $\ell_{\omega^{*}}^{2}(\mathbb{Z})$. In order to circumvent the fact that $h \in \mathcal{M}_{\omega_{(s)}}$ in the factorization $S^{k} u=\mathrm{e}^{h} * w$, it suffices to show that if $M$ is a nontrivial left-invariant subspace of $\ell_{\omega}^{2}(\mathbb{Z})$ and if $v \in \ell_{\omega^{*}}^{2}(\mathbb{Z})$ is orthogonal to $M$, then $v \in \ell_{\omega_{(s)}^{*}}^{2}(\mathbb{Z})$ for some $s<1 / 4$ (see the proof of Theorem 5.8). In order to do this, we will use the fact that $\left.u * v\right|_{\mathbb{Z}^{+}}=0$ for some nonzero $u \in \ell_{\omega}^{2}(\mathbb{Z})$. For $u \in \ell_{\omega}^{2}(\mathbb{Z})$, set $u^{+}(z)=\sum_{n=0}^{\infty} u_{n} z^{n}$ for $|z|<1, u^{-}(z)=\sum_{n<0} u_{n} z^{n}$ for $|z|>1$, and define in a similar way $v^{+}$and $v^{-}$for $v \in \ell_{\omega^{*}}^{2}(\mathbb{Z})$. The first step consists in constructing a suitable "Dynkin extension" [23] of $u^{-}$.

Let $\mu$ be a complex measure on $\mathbb{D}$, and let $C(\mu)(z)=\frac{1}{\pi} \iint_{\mathbb{D}} \frac{\mathrm{d} \mu(\xi)}{z-\xi}$ be the planar Cauchy transform of $\mu$, which is defined a.e. on $\mathbb{D}$ and holomorphic on $\mathbb{C} \backslash \overline{\mathbb{D}}$. Denote by $\mathcal{H}_{o}(\mathbb{C} \backslash \overline{\mathbb{D}})$ the space of holomorphic functions on $\mathbb{C} \backslash \overline{\mathbb{D}}$ vanishing at infinity. In the most general sense a Dynkin extension of $\varphi \in \mathcal{H}_{o}(\mathbb{C} \backslash \overline{\mathbb{D}})$ is a function $\psi \in L^{1}(\mathbb{D})$ such that $\psi=\left.C(\mu)\right|_{\mathbb{D}}, \varphi=\left.C(\mu)\right|_{\mathbb{C} \backslash \overline{\mathbb{D}}}$ for 
some complex measure $\mu$ on $\mathbb{D}$ (such a function $\psi$ exists if and only if there exists $f \in L^{1}(\mathbb{T}$ ) such that $\varphi(z)=\frac{1}{2 \mathrm{i} \pi} \int_{\mathbb{T}} \frac{f(\xi)}{\xi-z} \mathrm{~d} \xi$ for $|z|>1$, see [32]).

The first step, performed in Section 2, consists in using condition (2) to construct a Dynkin extension $D\left(u^{-}\right)$of $u^{-}$for $u \in \ell_{\omega}^{2}(\mathbb{Z})$ which has the two following properties:

$$
\begin{aligned}
& \bar{\partial} D\left(u^{-}\right) \in L^{1}(\mathbb{D}) ; \\
& v^{+} \cdot \bar{\partial} D\left(u^{-}\right) \in L^{1}(\mathbb{D}) \quad \text { for every } v \in \ell_{\omega}^{2}(\mathbb{Z}) .
\end{aligned}
$$

It seems that Dynkin extensions of this type can be found in an unpublished part of Dynkin's thesis, which was not accessible to the authors. The construction in Section 2, which is based on the two natural realizations of the dual of a weighted Bergman space on $\mathbb{D}$, is anyway a discrete analogue of the "extraction process" of Borichev-Hedenmalm [16] for the half-line.

Now set $U=u^{+}+D\left(u^{-}\right)$and let $v \in \ell_{\omega^{*}}^{2}(\mathbb{Z})$. Set $P^{+}\left(u^{+} v^{-}\right)(z)=\sum_{p=n+1}^{\infty} u_{p} v_{n-p} z^{n}$ for $z \in \mathbb{D}$.

We show in Theorem 3.1 that $v \perp S^{n} u$ for $n<0$ if and only if we have

$$
v^{+} . U=\left.\mathbb{C}\left(v^{+} . \bar{\partial} U\right)\right|_{\mathbb{D}}-P^{+}\left(u^{+} \cdot v^{-}\right) .
$$

In fact, formula (1.3) is essentially a version of the classical Cauchy-Pompeiu formula, see Remark 3.1.

In order to use formula (1.3) to obtain estimates on the growth of $v^{+}$when $v$ is orthogonal to some nontrivial left-invariant subspace of $\ell_{\omega}^{2}(\mathbb{Z})$ some control on the rate of decrease of $\bar{\partial} U(z)=\bar{\partial} D\left(u^{-}\right)(z)$ as $|z| \rightarrow 1^{-}$is needed. Such a control is given by Proposition 2.5, assuming that condition $\left(4^{\prime}\right)$ holds. Proposition 2.5 is a discrete version of some results of [16, Appendix B], but we prove it in Appendix A by a simple and direct method based on the inversion formula for Laplace transforms.

We now get to the crucial part of the proof. The growth of $P^{+}\left(u^{+} \cdot v^{-}\right)$can be controlled, and $\left.C\left(v^{+} . \bar{\partial} U\right)\right|_{\mathbb{D}} \in L^{1}(\mathbb{D})$ when $v$ and $u$ satisfy (1.3). When $\omega$ satisfies conditions (1), (2), (3), (4'), $\left(5^{\prime}\right)$, the function $U$ is asymptotically holomorphic in the disc, and Lemma 4.2 of [17] (stated in the paper as Lemma 5.2) provides lower bounds for $|u(z)|$ on a large class of circles centered at the origin. Using an averaging process involving these estimates it is possible to show that $v^{+} \cdot \bar{\partial} U$ is in fact bounded on $\mathbb{D}$. It is then possible to show that

$$
\limsup _{|z| \rightarrow 1^{-}} L_{\left(\omega^{*}\right)^{+}}^{-1}\left(|z|^{t}\right)\left|v^{+}(z)\right|<+\infty
$$

for some $t>4$, where we denote by $L_{\left(\omega^{*}\right)^{+}}$the Legendre transform of the weight $\left(\omega^{*}\right)^{+}=\omega_{\mid \mathbb{Z}^{+}}^{*}$. This shows that $v \in \ell_{\omega_{(s)}^{*}}$ for some $s<1 / 4$ if $v \in \ell_{\omega^{*}}^{2}(\mathbb{Z})$ is orthogonal to some nontrivial leftinvariant subspace fo $\ell_{\omega}^{2}(\mathbb{Z})$, and the result follows. Notice that these estimates on $v^{+}$were only a step in the proof of Theorem 5.8: it follows a posteriori from Theorem 5.8 that if $v$ is as above, then $\lim \sup _{n \rightarrow \infty}\left|v_{n}\right| / \omega_{+}^{(p)}(n)<+\infty$ for some $p \geqslant 1$, where $\left(\omega_{+}^{(p)}\right)_{p \geqslant 1}$ is the sequence of weights introducted in the discussion of Problem 1.

The situation is simpler when $\omega(n)=1$ for $n \geqslant 1$. In this case assume also that $\omega$ satisfies (1), (2), (4') and

$\left(5^{\prime}\right)\left(\frac{\log \omega(-n)}{\sqrt{ } n}\right)_{n \geqslant 1}$ is eventually increasing.

Using a corrected version of Lemma 4.7 of [18] (with the notations of [18], the hypothesis that $x \log \omega^{-1}(x)$ increases as $x$ decreases to 0 is used in the proof, but omitted in the statement of this lemma), it is possible to show directly that $v^{+}$belongs to the Nevanlinna class of the disc for every $v \in \ell_{\omega^{*}}^{2}(\mathbb{Z})$ which is orthogonal to a nontrivial left-invariant subspaces of $\ell_{\omega}^{2}(\mathbb{Z})$. 
This special case is developed in Section 4, and the proofs are somewhat simpler than in the general case, since in this situation $\ell_{\omega}^{2}(\mathbb{Z})$ is the space of Fourier sequences of a Hilbert space of functions in the circle. The results of Section 4 are closely related to the theorem of the summability of the logarithm, due to the second author [49,51].

The existence of nontrivial translation invariant subspaces of $\ell_{\omega}^{2}(\mathbb{Z})$ is an old-standing problem, and there were so far very few cases of concrete weights $\omega$ for which translation invariant subspaces of $\ell_{\omega}^{2}(\mathbb{Z})$ have been classified. It follows from Wiener's theorem [52] that the translation invariant subspaces of $\ell^{2}(\mathbb{Z})$ have the form $\widehat{X}_{E} * \ell^{2}(\mathbb{Z})$, where $X_{E}$ is the characteristic function of some Borel subset $E$ of $\mathbb{T}$. Also, for $r \in(0,1)$, set $\Omega_{r}=\{z \in \mathbb{C}|r<| z \mid<1\}$, and for $f \in \mathcal{H}\left(\Omega_{r}\right)$ denote by $\hat{f}(n)$ the $n$th Laurent coefficient of $f$. Since $H^{2}\left(\Omega_{r}\right)=\hat{\ell}_{\omega}^{2}(\mathbb{Z})$ when $\omega(n)=1$ for $n \geqslant 0, \omega(n)=r^{n}$ for $n<0$, it follows from Sarason's theorem [46] on the Hardy space $H^{2}\left(\Omega_{r}\right)$ that the translation invariant subspaces of $\ell_{\omega}^{2}(\mathbb{Z})$ have the form $\widehat{U} * \ell_{\omega}^{2}(\mathbb{Z})$, where $U$ is an "inner" function on the annulus $\Omega_{r}$ (see Section 4).

The results of Sections 4 and 5 provide a new class of concrete weights $\omega$ for which the translation invariant subspaces of $\ell_{\omega}^{2}(\mathbb{Z})$ can be classified. If, say, $\omega(n)=1$ for $n \geqslant 0$ and $\omega(n)=\mathrm{e}^{|n| /(1+\log |n|)}$ for $n<0$, then all nonzero translation invariant subspaces of $\ell_{\omega}^{2}(\mathbb{Z})$ have the form $\widehat{U} * \ell_{\omega}^{2}(\mathbb{Z})$, where $U$ is a singular inner function in the disc (see Theorem 4.6).

If $\omega(n)=(1+n)^{-1 / 2}$ for $n \geqslant 0, \omega(n)=\mathrm{e}^{|n| /(1+\log |n|)}$ for $n<0$, then all nonzero translation invariant subspaces of $\ell_{\omega}^{2}(\mathbb{Z})$ have the form $\widehat{U} * \ell_{\omega}^{2}(\mathbb{Z})$ where $U$ is a "singular inner function" of the Bergman space of the disc (Corollary 5.10).

More generally, if $\omega$ satisfies conditions (1), (2), (3), (4'), (5') then all nontrivial translation invariant subspaces of $\ell_{\omega}^{2}(\mathbb{Z})$ have the form $\bigvee_{n \leqslant 0} S^{n}(N)$, where $N$ is a nontrivial right-invariant subspace of $\ell_{\omega}^{2}\left(\mathbb{Z}^{+}\right)$having the division property.

Theorem 5.8 has also some strategic interest for the question of existence of nontrivial translation invariant subspaces of $\ell_{\omega}^{2}(\mathbb{Z})$. Denote by $\mathcal{S}^{+}$the class of weights $\sigma$ on $\mathbb{Z}^{+}$for which the spectrum of the shift and the spectrum of the backward shift on $\ell_{\omega}^{2}\left(\mathbb{Z}^{+}\right)$equal the closed unit disc. It is not difficult to check (see Section 6) that for every $\sigma \in \mathcal{S}^{+}$, there exists a weight $\omega$ on $\mathbb{Z}^{+}$satisfying conditions (1), (2), (3), (4'), (5') such that $\left.\omega\right|_{\mathbb{Z}^{+}}=\sigma$. The existence of nontrivial right-invariant subspaces of $\ell_{\omega}^{2}\left(\mathbb{Z}^{+}\right)$having the division property (or, equivalently, the existence of nontrivial, zero-free, $z$-invariant subspaces of index 1 ) in the weighted Hardy space $H_{\sigma}^{2}(\mathbb{D})$ is an open problem (Problem 3 in Section 6). A recent work of Atzmon [8,9] based on the theory of entire functions of zero exponential type, shows that such subspaces do exist when $\sigma$ is logconvex and satisfies a suitable regularity condition. Also, Borichev [15] showed that $H_{\sigma}^{2}(\mathbb{D})$ does have nontrivial zero-free $z$-invariant subspaces of index at least 2 if $\inf _{n \geqslant 0} \sigma(n)=0$, and Borichev, Hedenmalm and the second author constructed recently in [17] zero-free $z$-invariant subspaces of arbitrary index for all "large" Bergman spaces. We refer to the last section of [31] for a discussion of this problem (as mentioned above, it follows from Theorem 5.8 that a negative answer to Problem 3 would provide a counterexample to the hyperinvariant subspace problem for Hilbert spaces).

We give in Section 6 new examples of operators on Fréchet spaces without nontrivial hyperinvariant subspaces. Of course there are counterexamples to the invariant subspace problem for Fréchet spaces [5], but what is new here is that the spectrum of the operators given in Section 6 is the unit circle (the spectrum was empty for all previous counterexamples).

Notice that the existence of a nontrivial translation invariant subspace of $\ell_{\omega}^{2}(\mathbb{Z})$ is equivalent to the existence of $u \in \ell_{\omega}^{2}(\mathbb{Z}) \backslash\{0\}$ and $v \in \ell_{\omega^{*}}^{2}(\mathbb{Z}) \backslash\{0\}$ such that $u * v=0$. The results of Section 4 give a complete description of these pairs $(u, v)$ when, for example, $\omega(n)=1$ for $n \geqslant 0$ and $\omega(n)=\mathrm{e}^{|n| /(1+\log |n|)}$ for $n<0$. In this case, $v^{+}$belongs to the Nevanlinna class, the sum of 
the radial limits of $v^{+}$and $v^{-}$vanishes a.e. on $\mathbb{T}$, and $u \in \bigvee_{n \in \mathbb{Z}} S^{n} \widehat{V}$ where the singular inner function $V$ is the "denominator" of $v^{+}$(Corollary 4.7).

We refer to $[8-11,22,25,28]$ for recent contributions to the translation subspace problem for $\ell_{\omega}^{2}(\mathbb{Z})$. A positive answer was just obtained by Atzmon [10], using ideas related to Lomonosov's lemma for compact operators, for all symmetric weights.

The problem is still open even in the special case where the interior of the spectrum of $S$ is nonempty, despite partial results by Apostol [4] (see also [28]). It is also open when $\omega(n)$ $\omega(-n)=1$, despite recent progress obtained by Domar using entire functions of exponential type [22] (see also the related paper [29]).

Notice that the "continuous" analogue of the translation invariant subspace problem for $\ell_{\omega}^{2}(\mathbb{Z})$, i.e., the question of existence of nontrivial translation invariant subspaces for $L^{2}(\mathbb{R}, \omega)$, has been answered positively by Domar [21] (his simple and elegant argument also works for $\left.L^{p}(\mathbb{R}, \omega), 1 \leqslant p<+\infty\right)$. Domar's argument cannot be transferred to the discrete case (see [34] for a discussion of some links between the discrete and continuous cases). On the other hand, Domar's construction shows that there is no analogue of Theorem 5.8 for weights on $\mathbb{R}$. Domar's construction shows that there always exist nontrivial translation invariant subspaces $J$ of $L^{2}(\mathbb{R}, \omega)$ such that $f(x) \neq 0$ a.e. for every $f \in J \backslash\{0\}$, while Theorem 5.8 gives weights $\omega$ on $\mathbb{Z}$ such that $M \cap \ell_{\omega}^{2}\left(\mathbb{Z}^{+}\right) \neq\{0\}$ for every nontrivial translation invariant subspace $M$ of $\ell_{\omega}^{2}(\mathbb{Z})$.

The methods of this paper can be adapted, with minor modifications, to the spaces $\ell_{\omega}^{p}(\mathbb{Z})$, $1<p<+\infty$. The case $p=1$, which is significantly more complicated, has been considered by Harlouchet [33]. The authors wish to thank A. Atzmon, A. Borichev and N. Nikolskii for valuable discussions and exchange of informations when this work was completed. They also wish to thank the referee for bringing references [1] and [36] to their attention.

\section{Weighted Hardy and Bergman spaces, Dynkin extensions}

Denote by $\mathcal{S}^{+}$the set of weights $\sigma: \mathbb{Z}^{+} \rightarrow(0, \infty)$ satisfying the following conditions

$$
\begin{aligned}
& 0<\inf _{n \geqslant 0} \frac{\sigma(n+1)}{\sigma(n)} \leqslant \sup _{n \geqslant 0} \frac{\sigma(n+1)}{\sigma(n)}<+\infty, \\
& \lim _{n \rightarrow \infty} \bar{\sigma}(n)^{1 / n}=\lim _{n \rightarrow \infty} \tilde{\sigma}(n)^{1 / n}=1,
\end{aligned}
$$

where $\bar{\sigma}(n)=\sup _{p \geqslant 0} \frac{\sigma(p)}{\sigma(n+p)}, \tilde{\sigma}(n)=\sup _{p \geqslant 0} \frac{\sigma(n+p)}{\sigma(p)}(n \geqslant 0)$.

Denote by $\mathcal{H}(U)$ the space of holomorphic functions on an open subset $U$ of $\mathbb{C}$, and set

$$
\mathcal{H}_{0}(\mathbb{C} \backslash \overline{\mathbb{D}})=\{g \in \mathcal{H}(\mathbb{C} \backslash \overline{\mathbb{D}})|| g(\lambda) \mid \underset{|\lambda| \rightarrow \infty}{\rightarrow} 0\}
$$

For $f \in \mathcal{H}(\mathbb{D}), n \geqslant 0$ denote by $\hat{f}(n)$ the Taylor coefficient of order $n$ of $f$. Similarly for $g \in \mathcal{H}_{0}(\mathbb{C} \backslash \overline{\mathbb{D}}), n<0$ denote by $\hat{g}(n)$ the Laurent coefficient of order $n$ of $g$. Now let $\sigma \in \mathcal{S}^{+}$. Set

$$
\begin{aligned}
& \sigma^{*}(n)=\sigma^{-1}(-n-1) \quad(n<0), \\
& H_{\sigma}:=H_{\sigma}^{2}(\mathbb{D})=\left\{f \in \mathcal{H}(\mathbb{D}) \mid\|f\|_{\sigma}:=\left[\sum_{n=0}^{\infty}|\hat{f}(n)|^{2} \sigma^{2}(n)\right]^{1 / 2}<+\infty\right\}, \\
& H_{\sigma *}^{-}:=\left\{g \in \mathcal{H}_{0}(\mathbb{C} \backslash \overline{\mathbb{D}}) \mid\|g\|_{\sigma^{*}}:=\left[\sum_{n<0}|\hat{g}(n)|^{2} \sigma^{*}(n)^{2}\right]^{1 / 2}<+\infty\right\} .
\end{aligned}
$$


We can identify $H_{\sigma *}^{-}$to the dual of $H_{\sigma}$, the duality being implemented by the formula

$$
\langle f, g\rangle=\sum_{n=0}^{\infty} \hat{f}(n) \hat{g}(-n-1) \quad\left(f \in H_{\sigma}, g \in H_{\sigma *}^{-}\right) .
$$

We will denote by $z$ the identity map on $\mathbb{C}$. As usual, we will say that a closed subspace $M$ of $H_{\sigma}$ is $z$-invariant when $z M \subset M$. Now if $f \in H_{\sigma}, \lambda \in \mathbb{D}$ set

$$
f_{\lambda}(\xi)=\frac{f(\lambda)-f(\xi)}{\lambda-\xi}(\xi \in \mathbb{D} \backslash\{\lambda\}), \quad f_{\lambda}(\lambda)=f^{\prime}(\lambda) .
$$

As mentioned in the introduction the class $\mathcal{S}^{+}$is the class of weights on $\mathbb{Z}^{+}$for which the spectrum of the shift $f \rightarrow z f$ and the spectrum of the backward shift $f \rightarrow f_{o}$ is the closed unit disc.

We will use later the following notion.

Definition 2.1. - Let $\sigma \in \mathcal{S}^{+}$. A closed subspace $M$ of $H_{\sigma}$ has the division property if $f_{\lambda} \in M$ for every $f \in M$ and every $\lambda \in \mathbb{D}$ such that $f(\lambda)=0$.

We refer to [31] for a discussion of subspaces of $H_{\sigma}$ having the division property. Let $f \in H_{\sigma}$, $g \in H_{\sigma *}^{-}$. An immediate computation shows that we have

$$
\begin{aligned}
& |(\hat{f} * \hat{g})(n)| \leqslant\|f\|_{\sigma \cdot} \cdot\|g\|_{\sigma *} \cdot \tilde{\sigma}(-n-1) \quad(n<0), \\
& |(\hat{f} * \hat{g})(n)| \leqslant\|f\|_{\sigma \cdot} \cdot\|g\|_{\sigma *} \cdot \bar{\sigma}(n+1) \quad(n \geqslant 0) .
\end{aligned}
$$

Let $r \in(0,1)$. Set, for $f \in \mathcal{H}(\mathbb{D}), g \in \mathcal{H}_{0}(\mathbb{C} \backslash \overline{\mathbb{D}})$

$$
f_{r}(\xi)=f(r \xi) \quad\left(|\xi|<\frac{1}{r}\right), \quad g_{r}(\xi)=g\left(r^{-1} \xi\right) \quad(|\xi|>r)
$$

Clearly, $\left\|f_{r}-f\right\|_{\sigma} \rightarrow_{r \rightarrow 1^{-}} 0,\left\|g_{r}-g\right\|_{\sigma *} \rightarrow_{r \rightarrow 1^{-}} 0$, and we obtain, for $f \in H_{\sigma}, g \in H_{\sigma *}^{-}$,

$$
\langle f, g\rangle=\lim _{\substack{r \rightarrow 1^{-} \\ s \rightarrow 1^{-}}} \frac{1}{2 \mathrm{i} \pi} \int_{\mathbb{T}} f_{r}(\xi) g_{s}(\xi) \mathrm{d} \xi .
$$

Let $\lambda \in \mathbb{D}$. It follows from (2.9) that

$$
\sum_{n=0}^{\infty}(\hat{f} * \hat{g})(n) \lambda^{n}=\lim _{\substack{r \rightarrow 1^{-} \\ s \rightarrow 1^{-}}} \sum_{n=0}^{\infty} \hat{f}_{r} g_{s}(n) \lambda^{n}
$$

Also $\frac{1}{2 \mathrm{i} \pi} \int_{\mathbb{T}} \frac{F(\xi)}{\xi-\lambda} \mathrm{d} \xi=\sum_{n=0}^{\infty} \widehat{F}(n) \lambda^{n}$ for $F \in L^{1}(\mathbb{T})$, and so

$$
\sum_{n=0}^{\infty}(\hat{f} * \hat{g})(n) \lambda^{n}=\lim _{\substack{r \rightarrow 1^{-} \\ s \rightarrow 1^{-}}} \frac{1}{2 \mathrm{i} \pi} \int_{\mathbb{T}} \frac{f_{r}(\xi) g_{s}(\xi)}{\xi-\lambda} \mathrm{d} \xi
$$


Since $\int_{\mathbb{T}} \frac{g_{s}(\xi) \mathrm{d} \xi}{\xi-\lambda}=0, \frac{1}{2 \mathrm{i} \pi} \int_{\mathbb{T}} \frac{f_{r}(\xi) g_{s}(\xi)}{\xi-\lambda} \mathrm{d} \xi=\left\langle\left(f_{r}\right)_{\lambda}, g_{s}\right\rangle$. Since the map $f \rightarrow f_{\lambda}$ is continuous on $H_{\sigma}$, we obtain, for $\lambda \in \mathbb{D}, f \in H_{\sigma}, g \in H_{\sigma *}$

$$
\left\langle f_{\lambda}, g\right\rangle=\lim _{\substack{r \rightarrow 1^{-} \\ s \rightarrow 1^{-}}} \frac{1}{2 \mathrm{i} \pi} \int_{\mathbb{T}} \frac{f_{r}(\xi) g_{s}(\xi)}{\xi-\lambda} \mathrm{d} \xi=\sum_{n=0}^{\infty}(\hat{f} * \hat{g})(n) \lambda^{n} .
$$

We will say as usual that a sequence $\left(u_{n}\right)_{n \geqslant p}$ of strictly positive real numbers is log-convex when $u_{n+1}^{2} \leqslant u_{n} u_{n+2}$ for $n \geqslant p$. We will say that $\sigma: \mathbb{Z}^{+} \rightarrow(0, \infty)$ is log-convex if $(\sigma(n))_{n \geqslant 0}$ is log-convex, and we will say that $\sigma$ is eventually log-convex if $(\sigma(n))_{n \geqslant p}$ is log-convex for some $p \geqslant 0$. In this case, $\sigma \in \mathcal{S}^{+}$if and only if $\sigma(n+1) / \sigma(n) \rightarrow_{n \rightarrow \infty} 1$, which implies that $\sigma(n+1) \leqslant \sigma(n)$ for $n \geqslant p$. Set $L_{+}^{2}(0,1)=\left\{\varphi \in L^{2}(0,1) \mid \varphi(t)>0\right.$ a.e. $\}$. Denote by $\mathrm{d} m(\xi)$ the planar Lebesgue measure on $\mathbb{C}$ and let $\varphi \in L_{+}^{2}(0,1)$. Set

$$
\begin{gathered}
B_{\varphi}=B_{\varphi}^{2}(\mathbb{D})=\left\{f \in \mathcal{H}(\mathbb{D}) \mid\|f\|_{\varphi}:=\left[\frac{1}{\pi} \iint_{\mathbb{D}}|f(\xi)|^{2} \varphi^{2}(|\xi|) \mathrm{d} m(\xi)\right]^{1 / 2}<+\infty\right\}, \\
\sigma_{\varphi}(n)=\left[2 \int_{0}^{1} \varphi^{2}(t) t^{2 n+1} \mathrm{~d} t\right]^{1 / 2} \quad(n \geqslant 0) .
\end{gathered}
$$

Clearly, $\sigma_{\varphi} \in \mathcal{S}^{+}$is log-convex, and $\sigma_{\varphi}(n) \rightarrow_{n \rightarrow \infty} 0$. Conversely, if $\sigma \in \mathcal{S}^{+}$is eventually log-convex, and if $\sigma(n) \rightarrow_{n \rightarrow \infty} 0$ then there exists a function $\varphi \in L_{+}^{2}(0,1)$, continuous on $[0,1)$ such that

$$
0<\liminf _{n \rightarrow \infty} \frac{\sigma(n)}{\sigma_{\varphi}(n)} \leqslant \limsup _{n \rightarrow \infty} \frac{\sigma(n)}{\sigma_{\varphi}(n)}<+\infty .
$$

This follows from [14, Appendix A], see [23, Lemma 5.2]. Using polar coordinates, we obtain immediately, for $\varphi \in L_{+}^{2}(0,1)$

$$
B_{\varphi}=H_{\sigma_{\varphi}}, \quad\|f\|_{\varphi}=\|f\|_{\sigma_{\varphi}} \quad\left(f \in B_{\varphi}\right) .
$$

In order to give two interpretations of the dual of $B_{\varphi}$, we need to introduce the (planar) Cauchy transform, defined for $h \in L^{1}(\mathbb{D})$ by the formula

$$
\mathcal{C}(h)(\lambda)=\frac{1}{\pi} \iint_{\mathbb{D}} \frac{h(\xi)}{\lambda-\xi} \mathrm{d} m(\xi) .
$$

Then $\mathcal{C}(h) \in L_{\text {loc }}^{p}(\mathbb{C})$ for $p \in[1,2)$ (and $\mathcal{C}(h)$ is bounded and continuous on $\mathbb{C}$ if $\left.h \in \bigcup_{q>2} L^{q}(\mathbb{D})\right)$. Set $\mathcal{C}^{+}(h)=\left.\mathcal{C}(h)\right|_{\mathbb{D}}, \mathcal{C}^{-}(h)=\left.\mathcal{C}(h)\right|_{\mathbb{C} \backslash \overline{\mathbb{D}}}$.

We have $\bar{\partial} \mathcal{C}(h)(\lambda)=h(\lambda)$ a.e. on $\mathbb{D}$, and $\bar{\partial} \mathcal{C}(h)(\lambda)=0$ on $\mathbb{C} \backslash \overline{\mathbb{D}}$, the partial derivatives being taken in the sense of distribution theory, so obviously $\mathcal{C}^{-}(h) \in \mathcal{H}_{0}(\mathbb{C} \backslash \overline{\mathbb{D}})$.

Now let $\varphi \in L_{+}^{2}(0,1)$, and set $\varphi(\lambda)=\varphi(|\lambda|)$ for $\lambda \in \mathbb{D}$.

Notice that $\varphi^{2} \bar{B}_{\varphi} \subset \varphi^{2} \bar{B}_{\varphi} B_{\varphi} \subset L^{1}(\mathbb{D})$. Set

$$
[f, G]=\frac{1}{\pi} \iint_{\mathbb{D}} f(\xi) G(\xi) \mathrm{d} m(\xi) \quad\left(f \in B_{\varphi}, G \in \varphi^{2} \bar{B}_{\varphi}\right) .
$$

Clearly, there exists for every $g \in H_{\sigma_{\varphi}^{*}}^{-}$a unique $G \in \varphi^{2} \bar{B}_{\varphi}$ such that $[f, G]=\langle f, g\rangle$ for every $f \in B_{\varphi}$. This suggests the following definition. 
DEFINITION 2.2. - Let $\sigma \in \mathcal{S}^{+}$be an eventually log-convex weight such that $\sigma(n) \rightarrow_{n \rightarrow \infty} 0$. Set

$$
W(\sigma)=\left\{\varphi \in L_{+}^{2}(0,1) \mid 0<\liminf _{n \rightarrow \infty} \frac{\sigma(n)}{\sigma_{\varphi}(n)} \leqslant \limsup _{n \rightarrow \infty} \frac{\sigma(n)}{\sigma_{\varphi}(n)}<+\infty\right\} .
$$

For $g \in H_{\sigma *}^{-}, \varphi \in W(\sigma)$ we define the $\varphi$-Dynkin extension of $g$ to $\mathbb{D}$ by the formula

$$
\mathcal{D}_{\varphi}(g)=\mathcal{C}^{+}\left(\Delta_{\varphi}(g)\right)
$$

where $\Delta_{\varphi}(g) \in \varphi^{2} \bar{B}_{\varphi}$ is given by the relation

$$
\left[f, \Delta_{\varphi}(g)\right]=\langle f, g\rangle \quad\left(f \in H_{\sigma}\right) .
$$

From now on we will assume in this section that $\sigma \in \mathcal{S}^{+}$is eventually log-convex, and that $\sigma(n) \rightarrow_{n \rightarrow \infty} 0$.

Proposition 2.3. - Let $g \in H_{\sigma *}^{-}, \varphi \in W(\sigma)$ and set $G=\Delta_{\varphi}(g), \tilde{g}=\mathcal{D}_{\varphi}(g)$. We have, for $\lambda \in \mathbb{D}$ :

(i) $g=\mathcal{C}^{-}(G)$,

(ii) $G(\lambda)=\varphi^{2}(|\lambda|) \sum_{n=0}^{\infty} \hat{g}(-n-1) \sigma_{\varphi}^{-2}(n) \bar{\lambda}^{n}$,

(iii) $\tilde{g}$ is continuous on $\mathbb{D}$, and

$$
\tilde{g}(\lambda)=2 \sum_{n=0}^{\infty} \hat{g}(-n-1) \sigma_{\varphi}^{-2}(n) \lambda^{-n-1} \int_{0}^{|\lambda|} r^{2 n+1} \varphi^{2}(r) \mathrm{d} r
$$

(iv) $f(\lambda) \tilde{g}(\lambda)=\frac{1}{\pi} \iint_{\mathbb{D}} \frac{f(\xi) \bar{\partial} \tilde{g}(\xi)}{\lambda-\xi} \mathrm{d} m(\xi)+\left\langle f_{\lambda}, g\right\rangle\left(f \in H_{\sigma}\right)$.

Proof. - Denote again by $z$ the identity map on $\mathbb{D}$. It follows from (2.6) that we have $\hat{g}(n)=\left\langle z^{-n-1}, g\right\rangle$ for $n<0$.

Set $\theta_{\lambda}(\xi)=\frac{1}{\lambda-\xi}$ for $\xi \in \mathbb{D},|\lambda|>1$. We have $\theta_{\lambda}=\sum_{n=0}^{\infty} \lambda^{-n-1} z^{n}$, the series being convergent in $H_{\sigma}$, and so

$$
g(\lambda)=\left\langle\theta_{\lambda}, g\right\rangle \quad(|\lambda|>1)
$$

Hence

$$
g(\lambda)=\left[\theta_{\lambda}, G\right]=\frac{1}{\pi} \iint_{\mathbb{D}} \frac{G(\xi)}{\lambda-\xi} \mathrm{d} m(\xi) \quad \text { for }|\lambda|>1,
$$

which proves (i).

Set $F(\lambda)=\varphi^{-2}(|\lambda|) \cdot \bar{G}(\lambda)$ for $\lambda \in \mathbb{D}$, so that $F \in B_{\varphi}=H_{\sigma}$. We have, for $n \geqslant 0$

$$
\begin{aligned}
\hat{g}(-n-1) & =\left[z^{n}, G\right]=\frac{1}{\pi} \iint_{\mathbb{D}} \xi^{n} \cdot \varphi^{2}(|\xi|) \cdot \bar{F}(\xi) \mathrm{d} m(\xi) \\
& =\frac{1}{\pi} \int_{0}^{1} r^{2 n+1} \cdot \varphi^{2}(r)\left[\int_{0}^{2 \pi} \mathrm{e}^{\mathrm{i} n t} \cdot \bar{F}\left(r \mathrm{e}^{\mathrm{i} t}\right) \mathrm{d} t\right] \mathrm{d} r=\overline{\widehat{F}}(n) \cdot \sigma_{\varphi}^{2}(n) .
\end{aligned}
$$

Hence

$$
G(\lambda)=\varphi^{2}(|\lambda|) \cdot \sum_{n=0}^{\infty} \overline{\widehat{F}}(n) \cdot \bar{\lambda}^{n}=\varphi^{2}(|\lambda|) \cdot \sum_{n=0}^{\infty} \hat{g}(-n-1) \sigma_{\varphi}^{-2}(n) \bar{\lambda}^{n} \quad \text { for } \lambda \in \mathbb{D}
$$


wich proves (ii).

Set

$$
k_{n}(\lambda)=\varphi^{2}(|\lambda|) \bar{\lambda}^{n}, \quad \ell_{n}(\lambda)=2 \lambda^{-n-1} \int_{0}^{|\lambda|} r^{2 n+1} \varphi^{2}(r) \mathrm{d} r
$$

for $\lambda \in \mathbb{D}, n \geqslant 0$, so that $\left|\ell_{n}(\lambda)\right| \leqslant 2|\lambda|^{n} \cdot\|\varphi\|_{2}^{2}$.

The series $\sum_{n=0}^{\infty} \hat{g}(-n-1) \cdot \sigma_{\varphi}^{-2}(n) \ell_{n}$ converges uniformly on compact subsets of $\mathbb{D}$.

For $h \in L_{\text {loc }}^{1}(\mathbb{D})$ we have, in the sense of distribution theory,

$$
\bar{\partial} h\left(r \mathrm{e}^{\mathrm{i} t}\right)=\frac{\mathrm{e}^{\mathrm{i} t}}{2}\left[\frac{\partial h}{\partial r}\left(r \mathrm{e}^{\mathrm{i} t}\right)+\frac{\mathrm{i}}{r} \frac{\partial h}{\partial t}\left(r \mathrm{e}^{\mathrm{i} t}\right)\right] .
$$

Hence $\bar{\partial} \ell_{n}(\lambda)=\lambda^{-n-1} \cdot \frac{\lambda}{|\lambda|}|\lambda|^{2 n+1} \cdot \varphi^{2}(|\lambda|)=k_{n}(\lambda)$ a.e. on $\mathbb{D}$. Since $\ell_{n}$ extends continuously to $\overline{\mathbb{D}}$ we have, by the usual Cauchy-Pompeiu formula, for $\lambda \in \mathbb{D}$

$$
\ell_{n}(\lambda)=\frac{1}{\pi} \iint_{\mathbb{D}} \frac{k_{n}(\xi)}{\lambda-\xi} \mathrm{d} m(\xi)+\frac{1}{2 \mathrm{i} \pi} \int_{\mathbb{T}} \frac{\ell_{n}(\xi)}{\xi-\lambda} \mathrm{d} \xi
$$

But

$$
\int_{\mathbb{T}} \frac{\ell_{n}(\xi)}{\xi-\lambda} \mathrm{d} \xi=2 \sigma_{\varphi}^{2}(n) \int_{\mathbb{T}} \frac{\xi^{-n-1}}{\xi-\lambda} \mathrm{d} \xi=0,
$$

and so $\ell_{n}=\mathcal{C}^{+}\left(k_{n}\right)$. Since the map $f \rightarrow \varphi^{2} \cdot \bar{f}$ is continuous from $B_{\varphi}$ into $L^{1}(\mathbb{D})$, we have

$$
\left\|\tilde{g}-\sum_{n=0}^{p} \hat{g}(-n-1) \sigma_{\varphi}^{-2}(n) \ell_{n}\right\|_{L^{1}(\mathbb{D})} \underset{p \rightarrow \infty}{\rightarrow} 0 .
$$

Hence $\tilde{g}$ is continuous on $\mathbb{D}$, and

$$
\tilde{g}(\lambda)=2 \sum_{n=0}^{\infty} \hat{g}(-n-1) \sigma_{\varphi}^{-2}(n) \lambda^{-n-1} \int_{0}^{|\lambda|} r^{2 n+1} \varphi^{2}(r) \mathrm{d} r
$$

for $\lambda \in \mathbb{D}$, which proves (iii).

Now let $f \in H_{\sigma}, \lambda \in \mathbb{D}$. We have

$$
\begin{aligned}
\left\langle f_{\lambda}, g\right\rangle & =\left[f_{\lambda}, G\right]=\frac{1}{\pi} \iint_{\mathbb{D}} \frac{f(\lambda)-f(\xi)}{\lambda-\xi} G(\xi) \mathrm{d} m(\xi) \\
& =f(\lambda) \tilde{g}(\lambda)-\frac{1}{\pi} \iint_{\mathbb{D}} \frac{f(\xi) \bar{\partial} \tilde{g}(\xi)}{\lambda-\xi} \mathrm{d} m(\xi)
\end{aligned}
$$

which proves (iv).

Remark 2.1. - It follows from (2.12) that

$$
\left\langle f_{\lambda}, g\right\rangle=\sum_{n=0}^{\infty}(\hat{f} * \hat{g})(n) \lambda^{n} \quad \text { for } f \in H_{\sigma}, g \in H_{\sigma *}^{-}, \lambda \in \mathbb{D},
$$


and formula (iv) is essentially the Cauchy-Pompeiu formula: if $f \in \mathcal{C}^{1}(\overline{\mathbb{D}}) \cap \mathcal{H}(\mathbb{D})$, and if $\mathcal{C}(\bar{\partial} \tilde{g})$ is continuous on $\mathbb{C}$, then

$$
\sum_{n=0}^{\infty}(\hat{f} * \hat{g})(n) \lambda^{n}=\frac{1}{2 \mathrm{i} \pi} \int_{\mathbb{T}} \frac{f(\xi) \tilde{g}(\xi)}{\xi-\lambda} \mathrm{d} \xi
$$

and formula (iv) gives

$$
f(\lambda) \tilde{g}(\lambda)=\frac{1}{\pi} \iint_{\mathbb{D}} \frac{f(\xi) \bar{\partial} \tilde{g}(\xi)}{\lambda-\xi} \mathrm{d} m(\xi)+\frac{1}{2 \mathrm{i} \pi} \int_{\mathbb{T}} \frac{f(\xi) \tilde{g}(\xi)}{\xi-\lambda} \mathrm{d} \xi .
$$

Despite its simplicity, formula (iv) will play an important role in the paper.

We know that if $\sigma \in \mathcal{S}^{+}$is eventually log-convex, and if $\sigma(n) \rightarrow_{n \rightarrow \infty} 0$, then the set

$$
W(\sigma)=\left\{\phi \in L_{+}^{2}(0,1) \mid 0<\liminf _{n \rightarrow \infty} \frac{\sigma(n)}{\sigma_{\varphi}(n)} \leqslant \limsup _{n \rightarrow \infty} \frac{\sigma(n)}{\sigma_{\varphi}(n)}<+\infty\right\}
$$

contains a function $\phi$ which is continuous on $[0,1)$. We now give a simple condition on $\sigma$ which guarantees the existence of some $\phi \in W(\sigma)$ for which there is a good control on the rate of decrease of $\bar{\partial} \mathcal{D}_{\varphi}(g)(\lambda)$ as $|\lambda| \rightarrow 1^{-}$for every $g \in H_{\sigma *}$.

We will need the discrete form of the Legendre transform.

Definition 2.4. - Let $\sigma \in \mathcal{S}^{+}$. The Legendre transform of $\sigma$ is the function defined by the formula

$$
L_{\sigma}(r)=\sup _{n \geqslant 0} \frac{r^{n}}{\sigma(n)} \quad(r \in[0,1)) .
$$

Clearly, $L_{\sigma}(r) \rightarrow_{r \rightarrow 1^{-}} \infty$ if $\sigma(n) \rightarrow_{n \rightarrow \infty} 0$.

Now assume that $\sigma$ is log-convex. Set $r_{0}=0, r_{n}=\frac{\sigma(n)}{\sigma(n-1)}$ for $n \geqslant 1$. We have the following standard properties

$$
\begin{aligned}
& \frac{1}{\sigma(n)}=\inf _{0<r<1} L_{\sigma}(r) r^{-n} \quad(n \geqslant 0) ; \\
& L_{\sigma}(r)=\frac{r^{n}}{\sigma(n)} \quad\left(r_{n} \leqslant r \leqslant r_{n+1}, n \geqslant 0\right) ; \\
& r_{n}^{n} \geqslant \frac{\sigma(n)}{\sigma(0)} \quad(n \geqslant 1) .
\end{aligned}
$$

The following result is a discrete version of some results of [16, Appendix B]. We will give a direct proof in Appendix A.

Proposition 2.5. - Let $\sigma \in \mathcal{S}^{+}$. If the sequence $\left((n+1)^{\alpha} \sigma(n)\right)_{n \geqslant 0}$ is eventually logconvex for some $\alpha>3 / 2$, then $W(\sigma)$ contains a function $\phi$ satisfying the following conditions:

(i) $\phi$ is strictly decreasing and continuously differentiable on $[0,1)$.

(ii) For every $\delta \in(0,1-3 / 2 \alpha)$, there exists $k_{\delta}>0$ such that

$$
\left|\bar{\partial} \mathcal{D}_{\varphi}(g)(\lambda)\right| \leqslant k_{\delta} \cdot\|g\|_{\sigma *} \cdot L_{\sigma}^{-\delta}(|\lambda|) \quad\left(g \in H_{\sigma *}, \lambda \in \mathbb{D}\right) .
$$

Remark 2.2. - Set $\sigma_{\alpha}(n)=(n+1)^{\alpha} \sigma(n)$ for $n \geqslant 0$. If $\sigma \in \mathcal{S}^{+}$, and if $\sigma_{\alpha}$ is eventually log-convex for some $\alpha>1 / 2$, then $H_{\sigma *}^{-}$is a Banach algebra. This a discrete version of [16, Corollary 8.9], and the details can be found in [25, Proposition 2.16]. 


\section{Translation invariant subspaces of $\ell_{\omega}^{2}(\mathbb{Z})$, convolution equations on $\mathbb{Z}$, and a Cauchy-Pompeiu type formula}

We will denote by $\mathcal{S}$ the set of weights $\omega: \mathbb{Z} \rightarrow(0, \infty)$ satisfying the two following conditions

$$
\begin{gathered}
0<\inf _{n \in \mathbb{Z}} \frac{\omega(n+1)}{\omega(n)} \leqslant \sup _{n \in \mathbb{Z}} \frac{\omega(n+1)}{\omega(n)}<+\infty, \\
\tilde{\omega}(n)^{1 /|n|} \underset{|n| \rightarrow \infty}{\longrightarrow} 1, \quad \text { where } \tilde{\omega}(n):=\sup _{p \in \mathbb{Z}} \frac{\omega(n+p)}{\omega(p)}(n \in \mathbb{Z}) .
\end{gathered}
$$

For $\omega \in \mathcal{S}$, set

$$
\begin{aligned}
& \ell_{\omega}:=\ell_{\omega}^{2}(\mathbb{Z})=\left\{u=\left(u_{n}\right)_{n \in \mathbb{Z}} \mid\|u\|_{\omega}=\left[\sum_{n \in \mathbb{Z}}\left|u_{n}\right|^{2} \omega^{2}(n)\right]^{1 / 2}<+\infty\right\}, \\
& \omega^{*}(n)=\omega^{-1}(-n-1) \quad(n \in \mathbb{Z}), \quad \omega_{+}=\left.\omega\right|_{\mathbb{Z}^{+}}, \quad \omega_{-}=\left.\omega\right|_{\mathbb{Z}^{-}},
\end{aligned}
$$

where we denote by $\mathbb{Z}^{+}$the set of nonnegative integers and $\mathbb{Z}^{-}$the set of negative integers. Clearly, $\mathcal{S}^{+}=\left\{\omega_{+}\right\}_{\omega \in \mathcal{S}}$, where $\mathcal{S}^{+}$is the set introduced in Section 2 .

The usual bilateral shift operator $S$ on $\ell_{\omega}$ is defined by the formula

$$
S u=\left(u_{n-1}\right)_{n \in \mathbb{Z}} \quad\left(u=\left(u_{n}\right)_{n \in \mathbb{Z}} \in \ell_{\omega}\right) .
$$

The operator $S$ is bounded and invertible on $\ell_{\omega}$, and we have

$$
\left\|S^{n}\right\|=\tilde{\omega}(n) \quad(n \in \mathbb{Z})
$$

so that $\operatorname{Spec}(S)=\mathbb{T}$.

The dual of $\ell_{\omega}$ can be identified to $\ell_{\omega *}$, the duality being implemented by the formula

$$
\langle u, v\rangle=\sum_{p \in \mathbb{Z}} u_{p} v_{-p-1} \quad\left(u=\left(u_{n}\right)_{n \in \mathbb{Z}} \in \ell_{\omega}, v=\left(v_{n}\right)_{n \in \mathbb{Z}} \in \ell_{\omega *}\right)
$$

Now set

$$
\mathcal{E}=\left\{u=\left.\left(u_{n}\right)_{n \in \mathbb{Z}}\left|\varlimsup_{|n| \rightarrow \infty}\right| u_{n}\right|^{1 /|n|} \leqslant 1\right\} .
$$

We have for $u=\left(u_{n}\right)_{n \in \mathbb{Z}} \in \ell_{\omega}, v=\left(v_{n}\right)_{n \in \mathbb{Z}} \in \ell_{w *}$

$$
\left|\sum_{p \in \mathbb{Z}} u_{p} v_{n-p}\right| \leqslant\|u\|_{\omega} \cdot\left\|v_{n}\right\|_{\omega *} \tilde{\omega}(-n-1) \quad(n \in \mathbb{Z})
$$

and so $u * v:=\left(\sum_{p \in \mathbb{Z}} u_{p} v_{n-p}\right)_{n \in \mathbb{Z}} \in \mathcal{E}$.

Denote by $H F(\mathbb{T})$ the set of hyperfunctions on the unit circle $\mathbb{T}$, i.e. the set of all pairs $F=(f, g)$ where $f \in \mathcal{H}(\mathbb{D}), g \in \mathcal{H}_{0}(\mathbb{C} \backslash \overline{\mathbb{D}})$. Denote by $P^{+}: H F(\mathbb{T}) \rightarrow \mathcal{H}(\mathbb{D})$ the map $(f, g) \rightarrow f$ and denote by $P^{-}: H F(\mathbb{T}) \rightarrow \mathcal{H}_{0}(\mathbb{C} \backslash \overline{\mathbb{D}})$ the map $(f, g) \rightarrow g$. The notations being as in Section 2, set, for $F \in H F(\mathbb{T})$

$$
\widehat{F}(n)=\widehat{P^{+}(F)}(n) \quad(n \geqslant 0), \quad \widehat{F}(n)=\widehat{P^{-}(F)}(n) \quad(n<0) .
$$

Also for $u \in \mathcal{E}$ set

$$
\tilde{u}=\left(u^{+}, u^{-}\right),
$$


where $u^{+}$and $u^{-}$are defined by the formula

$$
\begin{array}{ll}
u^{+}(\lambda)=\sum_{n=0}^{\infty} \lambda^{n} u_{n} & (|\lambda|<1), \\
u^{-}(\lambda) & =\sum_{n<0} \lambda^{n} u_{n} \quad(|\lambda|>1) .
\end{array}
$$

The Fourier transform $\mathcal{F}: F \rightarrow \widehat{F}:=(\widehat{F}(n))_{n \in \mathbb{Z}}$ is a bijection from $H F(\mathbb{T})$ onto $\mathcal{E}$, and $\mathcal{F}^{-1}(u)=\tilde{u}$ for $u \in \mathcal{E}$ (the hyperfunction $\tilde{u}$ is often called the Carleman transform of $u$ ).

We will often identify a function $F \in L^{1}(\mathbb{T})$ to the hyperfunction having the same Fourier coefficients. We obtain, for $F \in L^{1}(\mathbb{T})$

$$
\begin{aligned}
& P^{+}(F)(\lambda)=\frac{1}{2 \mathrm{i} \pi} \int_{\mathbb{T}} \frac{F(\xi)}{\xi-\lambda} \mathrm{d} \xi \quad(|\lambda|<1), \\
& P^{-}(F)(\lambda)=-\frac{1}{2 \mathrm{i} \pi} \int_{\mathbb{T}} \frac{F(\xi)}{\xi-\lambda} \mathrm{d} \xi \quad(|\lambda|>1),
\end{aligned}
$$

and so, by the Plemelj-Privalov formula

$$
F(\xi)=\lim _{r \rightarrow 1^{-}} P^{+}(F)(r \xi)+\lim _{r \rightarrow 1^{-}} P^{-}(F)\left(r^{-1} \xi\right) \quad \text { a.e. on } \mathbb{T} \text {. }
$$

Now let $\omega \in \mathcal{S}$. For $u \in \ell_{\omega}, v \in \ell_{\omega *}$ we define the "product" $\tilde{u} \tilde{v}$ by the formula

$$
\tilde{u} \tilde{v}=\widetilde{u * v} .
$$

Identifying $u^{+}$to the hyperfunction $\left(u^{+}, 0\right), u^{-}$to the hyperfunction $\left(0, u^{-}\right)$, etc. we can use formula (3.17) to define the products $u^{+} v^{-}$and $v^{+} u^{-}$for $u \in \ell_{\omega}, v \in \ell_{\omega *}$. Notice that if $\omega(n)=1$ for $n \geqslant 0$ then $u^{+} \in H^{2}(\mathbb{D}), v^{-} \in H^{2}(\mathbb{C} \backslash \mathbb{D})$, and so $u^{+} v^{-}$can be considered as an element of $L^{1}(\mathbb{T})$. We obtain in this case, for $u \in \ell_{\omega *}$

$$
P^{+}\left(u^{+} v^{-}\right)(\lambda)=\frac{1}{2 \mathrm{i} \pi} \int_{\mathbb{T}} \frac{u^{+}(\xi) v^{-}(\xi)}{\xi-\lambda} \mathrm{d} \xi \quad(|\lambda|<1) .
$$

For $\omega \in \mathcal{S}$, set

$$
\begin{aligned}
& \ell_{\omega}^{+}=\left\{u=\left(u_{n}\right)_{n \in \mathbb{Z}} \in \ell_{\omega} \mid u_{n}=0(n<0)\right\}, \\
& \ell_{\omega}^{-}=\left\{u=\left(u_{n}\right)_{n \in \mathbb{Z}} \in \ell_{\omega} \mid u_{n}=0(n \geqslant 0)\right\} .
\end{aligned}
$$

We can identify $\ell_{\omega}^{+}$to

$$
\ell_{\omega_{+}}\left(\mathbb{Z}^{+}\right)=\left\{u=\left.\left(u_{n}\right)_{n \geqslant 0}\left|\sum_{n=0}^{\infty}\right| u_{n}\right|^{2} \omega^{2}(n)<+\infty\right\} .
$$

The Fourier transform is then an isometry from the weighted Hardy space $H_{\omega_{+}}$onto $\ell_{\omega}^{+}$, which defines a unitary equivalence between the operator of multiplication by $z$ on $H_{\omega_{+}}$and $S^{+}:=\left.S\right|_{\ell_{\omega}^{+}}$.

Recall that a closed subspace $M$ of $\ell_{\omega}$ is said to be translation invariant (resp. right-invariant, resp. left-invariant) when $S(M)=M\left(\operatorname{resp} . S(M) \subset M\right.$, resp. $\left.S^{-1}(M) \subset M\right)$. Such a subspace 
is said to be nontrivial when $M \neq\{0\}, M \neq \ell_{\omega}$. We will use the standard notation $V A$ to denote the closed linear span of $A \subset \ell_{\omega}$. Let $u \in \ell_{\omega}, v \in \ell_{\omega *}$. Clearly, $v \perp \bigvee_{n \geqslant 0} S^{n} u$ if and only if $\left.u * v\right|_{\mathbb{Z}^{-}}=0, v \perp \bigvee_{n<0} S^{n} u$ if and only if $\left.u * v\right|_{\mathbb{Z}^{+}}=0$, and $v \perp \bigvee_{n \in \mathbb{Z}} S^{n} u$ if and only if $u * v=0$, and the existence of a nontrivial translation invariant subspace of $\ell_{\omega}$ is equivalent to the existence of $u \in \ell_{\omega} \backslash\{0\}$ and $v \in \ell_{\omega *} \backslash\{0\}$ such that $u * v=0$.

Recall that a sequence $\left(a_{n}\right)_{n \geqslant p}$ of positive real numbers is said to be log-concave if the sequence $\left(a_{n}^{-1}\right)_{n \geqslant p}$ is $\log$-convex. In particular, if $\omega \in \mathcal{S}$, the fact that the sequence $(\omega(-n))_{n \geqslant 1}$ is eventually log-concave means that $\omega_{+}^{*}$ is eventually log-convex. In this situation, the space $\left\{v^{+}\right\}_{v \in \ell_{\omega *}}$ is the weighted Hardy space $H_{\omega_{+}^{*}}$ and the space $\left\{u^{-}\right\}_{u \in \ell_{\omega}}$ is the space $H_{\left(\omega_{+}^{*}\right)^{*}}^{-}=H_{\omega_{-}}^{-}$. Hence we can construct the Dynkin extension $\mathcal{D}_{\varphi}\left(u^{-}\right)$for every $u \in \ell_{\omega}$ if $\varphi \in W\left(\omega_{+}^{*}\right)$.

As in Section 2 we denote by $\mathcal{C}^{+}(F)$ the restriction to the disc of the planar Cauchy transform of $F \in L^{1}(\mathbb{D})$. The following simple result will play a basic role in the next sections.

THEOREM 3.1. - Let $\omega \in \mathcal{S}$, and assume that the sequence $(\omega(-n))_{n \geqslant 1}$ is eventually logconcave. Let $\varphi \in W\left(\omega_{+}^{*}\right), u \in \ell_{\omega}, v \in \ell_{\omega *}$. Then the two following conditions imply each other

(i) $v \perp \bigvee_{n<0} S^{n} u$,

(ii) $v^{+}\left(u^{+}+\mathcal{D}_{\varphi}\left(u^{-}\right)\right)=\mathcal{C}^{+}\left(v^{+} \bar{\partial} \mathcal{D}_{\varphi}\left(u^{-}\right)\right)-P^{+}\left(u^{+} v^{-}\right)$.

Proof. - It follows from formula (2.12) and Proposition 2.3 that we have, for $\lambda \in \mathbb{D}$,

$$
v^{+}(\lambda) \mathcal{D}_{\varphi}\left(u^{-}\right)(\lambda)=\frac{1}{\pi} \iint_{\mathbb{D}} \frac{v^{+}(\xi) \bar{\partial} \mathcal{D}_{\varphi}\left(u^{-}\right)(\xi)}{\lambda-\xi} \mathrm{d} m(\xi)+\sum_{n=0}^{\infty}\left(\widehat{u^{-}} * \widehat{v^{+}}\right)(n) \lambda^{n} .
$$

Set $F=v^{+}\left(u^{+}+\mathcal{D}_{\varphi}\left(u^{-}\right)\right)+P^{+}\left(u^{+} v^{-}\right)-\mathcal{C}\left(v^{+} \bar{\partial} \mathcal{D}_{\varphi}\left(u^{-}\right)\right)$. Then $F \in \mathcal{H}(\mathbb{D})$ and we have

$$
\widehat{F}=\left.\widehat{u^{+}} * \widehat{v^{+}}\right|_{\mathbb{Z}^{+}}+\left.\widehat{u^{+}} * \widehat{v^{-}}\right|_{\mathbb{Z}^{+}}+\left.\widehat{u^{-}} * \widehat{v^{+}}\right|_{\mathbb{Z}^{+}}
$$

But $\left.u * v=\left(\widehat{u^{+}}+\widehat{u^{-}}\right) * \widehat{\left(v^{+}\right.}+\widehat{v^{-}}\right)$, and $\left.\widehat{u^{-}} * \widehat{v^{-}}\right|_{\mathbb{Z}^{+}}=\{0\}$. Hence $\widehat{F}=\left.u * v\right|_{\mathbb{Z}^{+}}$, and the result follows.

Remark 3.1. - (i) Heuristically, $-P^{+}\left(u^{+} v^{-}\right)$represents the Cauchy integral of the "boundary value" of $v^{+}\left(u^{+}+\mathcal{D}_{\varphi}\left(u^{-}\right)\right)$on $\mathbb{T}$, and condition (ii) of Theorem 3.1 is again a version of the classical Cauchy-Pompeiu formula.

(ii) If $\omega(n)=1$ for $n \geqslant 0$ we can use formula (3.18) and condition (ii) of Theorem 3.1 gives, for $\lambda \in \mathbb{D}$

$$
\begin{aligned}
& v^{+}(\lambda)\left[u^{+}(\lambda)+\mathcal{D}_{\varphi}\left(u^{-}\right)(\lambda)\right] \\
& =\frac{1}{\pi} \iint_{\mathbb{D}} \frac{v^{+}(\xi) \bar{\partial} \mathcal{D}_{\varphi}\left(u^{-}\right)(\xi)}{\lambda-\xi} \mathrm{d} m(\xi)+\frac{1}{2 \mathrm{i} \pi} \int_{\mathbb{T}} \frac{u^{+}(\xi) v^{-}(\xi)}{\lambda-\xi} \mathrm{d} \xi
\end{aligned}
$$

In the next two sections we shall show that if the sequence $(\omega(-n))_{n \geqslant 0}$ grows "sufficiently fast and regularly" then all left-invariant subspaces of $\ell_{\omega}$ have the form $\bigvee_{n \leqslant-k} S^{n} \widehat{M}$ for some $k \geqslant 0$, where $M$ is a closed subspace of $H_{\omega_{+}}$having the "division property" introduced in Section 2. In particular all translation invariant subspaces of $\ell_{\omega}$ have the form $\bigvee_{n \leqslant 0} S^{n} \widehat{M}$ for some $z$-invariant subspace $M$ of $H_{\omega_{+}}$having the division property. 


\section{The case $\omega(n)=1$ for $n \geqslant 0$}

In this section we restrict attention to the case where $\omega(n)=1$ for $n \geqslant 0$ and where $\omega(-n) \rightarrow_{n \rightarrow \infty} \infty$. In this situation, set

$$
L_{\omega}^{2}(\mathbb{T})=\left\{f \in L^{2}(\mathbb{T}) \mid\|f\|_{\omega}:=\left[\sum_{n \in \mathbb{Z}}|\hat{f}(n)|^{2} \omega^{2}(n)\right]^{1 / 2}<+\infty\right\} .
$$

Then $L_{\omega}^{2}(\mathbb{T})=H^{2} \oplus H_{\omega^{-}}^{-}$, where we denote by $H^{2}=H^{2}(\mathbb{D})$ the usual Hardy space, and $\ell_{\omega}=\widehat{L_{\omega}^{2}(\mathbb{T})}$. Hence the shift $S$ on $\ell_{\omega}$ is unitarily equivalent to the multiplication operator $T$ defined by the formula

$$
T(f)\left(\mathrm{e}^{\mathrm{i} t}\right)=\mathrm{e}^{\mathrm{i} t} f\left(\mathrm{e}^{\mathrm{i} t}\right) \quad \text { a.e. on } \mathbb{T}\left(f \in L_{\omega}^{2}(\mathbb{T})\right) .
$$

We will say that a closed subspace $M$ of $L_{\omega}^{2}(\mathbb{T})$ is $z$-invariant (resp. $z^{-1}$-invariant, resp. $z$-biinvariant) if $T(M) \subset M$ (resp. $T^{-1}(M) \subset M, \operatorname{resp} . T(M)=M$ ) and we will use the notation $T^{n}(M)=z^{n} . M$ for $M \subset L_{\omega}^{2}(\mathbb{T}), n \in \mathbb{Z}$. The existence of nontrivial $z$-biinvariant subspaces of $L_{\omega}^{2}(\mathbb{T})$ was pointed out only recently [25, Theorem 5-7]: if $\omega(n)=1$ for $n \geqslant 0$, and if $\omega(n) \rightarrow_{n \rightarrow \infty} \infty$ then there exists a singular inner function $U$ such that $\bigvee_{n \in \mathbb{Z}} z^{n} U \varsubsetneqq L_{\omega}^{2}(\mathbb{T})$. If, further, $\frac{\log \omega(-n)}{\sqrt{n}} \rightarrow_{n \rightarrow \infty} \infty$ then $\bigvee_{n \in \mathbb{Z}} z^{n} U \varsubsetneqq L_{\omega}^{2}(\mathbb{T})$ for every singular inner function $U$ [25, Theorem 3-8]. These facts are related to a trivial convolution equation. If

$$
U=U_{\mu}: z \rightarrow \exp \left[-\frac{1}{2 \pi} \int_{-\pi}^{\pi} \frac{\mathrm{e}^{\mathrm{i} t}+z}{\mathrm{e}^{\mathrm{i} t}-z} \mathrm{~d} \mu(t)\right]
$$

is the singular inner function associated to a positive singular measure $\mu$ on $\mathbb{T}$, extend $U$ to $\mathbb{C} \backslash \overline{\mathbb{D}}$ by using the same formula and set

$$
\begin{array}{ll}
U^{*}(\lambda)=U^{-1}(\lambda)-U(0) & (|\lambda|<1) \\
U^{*}(\lambda)=-U^{-1}(\lambda)+U(0) & (|\lambda|>1) .
\end{array}
$$

Then $U^{*} \in H F(\mathbb{T})$ and, identifying $\bar{U} \in L^{2}(\mathbb{T})$ to the hyperfunction having the same Fourier coefficients, we have $U^{*}=\left(U^{-1}, 0\right)-\bar{U}$, so that $\widehat{U} * \widehat{U}^{*}=0$. Hence $\bigvee_{n \in \mathbb{Z}} S^{n} \widehat{U} \varsubsetneqq L_{\omega}^{2}(\mathbb{T})$ if $U^{*} \in \ell_{\omega^{*}}$, which is indeed the case if $(\log \omega(-n)) / \sqrt{n} \rightarrow_{n \rightarrow \infty} \infty$. Assume again that $\omega(n)=1$ for $n \geqslant 0$, and that $\liminf _{n \rightarrow \infty} \omega(-n)>0$. We will say that $\omega$ is quasianalytic when we have

$$
\sum_{n<0} \frac{\log \omega(n)}{n^{2}}=+\infty
$$

If $\omega$ is not quasianalytic, it follows from the discrete version of the Beurling-Malliavin theorem [13] that $M_{V} \neq\{0\}$ for every nonempty open $\operatorname{arc} V$, where

$$
M_{V}=\left\{f \in L_{\omega}^{2}(\mathbb{T})|f|_{V}=0 \text { a.e. }\right\},
$$

which provides another family of nontrivial $z$-biinvariant subspaces of $L_{\omega}^{2}(\mathbb{T})$.

On the other hand if $\sum_{n=1}^{\infty} \frac{\log \rho(n)}{n^{2}}=+\infty$, where $\rho(n)=\left[\sum_{p \leqslant-n} \frac{1}{\omega^{2}(p)}\right]^{-1}$, a sharp result of Beurling [12, p. 407] shows that $f\left(\mathrm{e}^{\mathrm{i} t}\right) \neq 0$ a.e. for every nonzero $f \in L_{\omega}^{2}(\mathbb{T})$. 
In particular if $\omega$ is quasianalytic, and if the sequence $\left(\omega(-n) / n^{\alpha}\right)_{n \geqslant 1}$ is eventually increasing for some $\alpha>1 / 2$, then $f\left(\mathrm{e}^{\mathrm{i} t}\right) \neq 0$ a.e. for every nonzero $f \in L_{\omega}^{2}(\mathbb{T})$. The now classical "Theorem of summability of the logarithm" $[18,49-51]$, due to the second author, says much more: we have $\log |f| \in L^{1}(\mathbb{T})$ for every nonzero $f \in L_{\omega}^{2}(\mathbb{T})$ if $\omega$ is quasianalytic, with $\omega(n)=1$ for $n \geqslant 0$, and satisfies the two following regularity conditions

$$
\begin{aligned}
& (\omega(-n))_{n \geqslant 1} \text { is eventually log-concave, } \\
& \left(\frac{\log \omega(-n)}{\sqrt{n}}\right)_{n \geqslant 1} \text { is eventually increasing. }
\end{aligned}
$$

In the remainder of this section we will consider quasianalytic weights $\omega$, with $\omega(n)=1$ for $n \geqslant 0$ which satisfy (4.6) and

$$
\left(\frac{\omega(-n)}{n^{\alpha}}\right)_{n \geqslant 1} \text { is eventually log-concave for some } \alpha>\frac{3}{2} .
$$

Denote by $|A|$ the Lebesgue measure of a Borel set $A \subset \mathbb{R}$ and notice that if $F \in \mathcal{C}^{1}(\mathbb{D}) \cap$ $L^{\infty}(\mathbb{D})$ and if $\bar{\partial} F \in L^{\infty}(\mathbb{D})$, then $\mathcal{C}(\bar{\partial} F)$ is continuous on $\mathbb{C}, F-\mathcal{C}^{+}(\bar{\partial} F) \in H^{\infty}$, so that $\lim _{r \rightarrow 1^{-}} F\left(r \mathrm{e}^{\mathrm{i} t}\right)$ exists a.e. on $\mathbb{T}$. We now state as a lemma two important results concerning asymptotically holomorphic functions in the disc.

LEMmA 4.1 [17, Lemma 4.7 and Theorem 5.10]. - Let $F \in \mathcal{C}^{1}(\mathbb{D}) \cap L^{\infty}(\mathbb{D}), r_{0} \in[0,1)$ and let $\rho:\left[r_{0}, 1\right) \rightarrow(0, \infty)$ be a continuous function such that $(1-r) \log \rho(r)$ is increasing on $\left[r_{0}, 1\right)$ and such that

$$
\int_{r_{0}}^{1} \log \log \rho(r) \mathrm{d} r=+\infty .
$$

Assume that $|\bar{\partial} F(\lambda)| \leqslant \rho^{-1}(|\lambda|)$ for $r_{0} \leqslant|\lambda|<1$.

Then either $\lim _{r \rightarrow 1^{-}} F\left(r \mathrm{e}^{\mathrm{i} t}\right)=0$ a.e. or $F$ satisfies the two following conditions:

(i) There exists $k_{0} \geqslant 1$ such that we have, for $k \geqslant k_{0}$

$$
\left|\left\{r \in\left[1-2^{-k}, 1-2^{-k-1}\right]\left|\inf _{|\lambda|=r}\right| F(\lambda) \mid>\rho^{-1}(|\lambda|)\right\}\right| \geqslant 2^{-k-2}
$$

(ii) $\varlimsup_{r \rightarrow 1^{-}} \int_{0}^{2 \pi} \log \left|F\left(r \mathrm{e}^{\mathrm{i} t}\right)\right| \mathrm{d} t>-\infty$.

An important result of Bourgain [19] shows that a function $f \in L^{2}(\mathbb{T}) \backslash\{0\}$ belongs to $H^{2} \cdot \bar{H}^{\infty}$ if and only if $\log |f| \in L^{1}(\mathbb{T})$. Lemma 4.1 gives more precise factorizations for $f \in L_{\omega}^{2}(\mathbb{T}) \backslash\{0\}$ when $\omega$ satisfies the hypothesis of the theorem of summability of the logarithm. Set $H_{0}^{2}=\left\{f \in H^{2} \mid f(0)=0\right\}$. We state as a corollary such a result, which follows from the proof of [18, Theorem 6.3]. A more precise result will be given in Appendix B.

COROLlary 4.2. - Let $\omega \in \mathcal{S}$ be a quasianalytic weight satisfying (4.5) and (4.6), with $\omega(n)=1$ for $n \geqslant 0$. Then for every $f \in L_{\omega}^{2}(\mathbb{T})$ there exists $g \in H^{2}, h \in H_{0}^{2}, k \geqslant 0$ satisfying the following conditions:

(i) $\left(\log ^{-}|\hat{h}(n)|\right) / \sqrt{n} \rightarrow_{n \rightarrow \infty} \infty$;

(ii) $f\left(\mathrm{e}^{\mathrm{i} t}\right)=\mathrm{e}^{-\mathrm{i} k t} \mathrm{e}^{\bar{h}\left(\mathrm{e}^{\mathrm{i} t}\right)} g\left(\mathrm{e}^{\mathrm{i} t}\right)$ a.e. on $\mathbb{T}$.

Notice that the theorem of summability of the logarithm is an immediate consequence of Corollary 4.2. 
We follow now the strategy outlined in the introduction. Denote by

$$
\begin{aligned}
\mathcal{N}(\mathbb{D}) & =\left\{f \in \mathcal{H}(\mathbb{D})\left|\sup _{0 \leqslant r<1} \int_{0}^{2 \pi} \log ^{+}\right| f\left(r \mathrm{e}^{\mathrm{i} t}\right) \mid \mathrm{d} t<+\infty\right\} \\
& =\left\{f \in \mathcal{H}(\mathbb{D})\left|\underline{\lim }_{r \rightarrow 1^{-}} \int_{0}^{2 \pi} \log ^{+}\right| f\left(r \mathrm{e}^{\mathrm{i} t}\right) \mid \mathrm{d} t<+\infty\right\}
\end{aligned}
$$

the Nevanlinna class of the disc.

LEMMA 4.3. - Let $\omega \in \mathcal{S}$ be a quasianalytic weight such that $\omega(n)=1$ for $n \geqslant 0$, and assume that $\omega$ satisfies the regularity conditions (4.6) and (4.7). Then $v^{+} \in \mathcal{N}(\mathbb{D})$ for every $v \in \ell_{\omega^{*}}$ which is not left-cyclic.

Proof. - For $\sigma \in \mathcal{S}^{+}$denote again by $L_{\sigma}$ the Legendre transform of $\sigma$ introduced in Definition 2.3. We have, for $c>0$

$$
L_{\sigma^{1 / c}}(r)=L_{\sigma}\left(r^{c}\right)^{1 / c} \quad(0 \leqslant r<1) .
$$

Now set $\sigma(n)=\omega(-n-1)^{-1}$ for $n \geqslant 0$, so that $\sigma=\left(\omega^{*}\right)_{+}$, according to the notations of Section 3. Then $\sigma$ is eventually $\log$-convex and since $\sum_{n=1}^{\infty} \frac{\log \sigma(n)}{n^{2}}=+\infty$, we have (see [12])

$$
\int_{0}^{1} \log \log L_{\sigma}(r) \mathrm{d} r=+\infty
$$

Also, since the sequence $\left(\left(\log \sigma^{-1 / c}(n)\right) / \sqrt{n}\right)_{n \geqslant 1}$ is eventually increasing, it follows from [43] and (4.8) that we have, for $c>0$

$$
(1-r) \log L_{\sigma}\left(r^{c}\right) \text { is eventually increasing as } r \rightarrow 1^{-} .
$$

Let $u \in \ell_{\omega} \backslash\{0\}$ such that $(u * v)_{n}=0$ for $n \geqslant 0$, and let $\phi \in W(\sigma)$ satisfy the conditions of Proposition 2.5. Denote by $\mathcal{D}_{\varphi}\left(u^{-}\right)$the $\varphi$-Dynkin extension of $u^{-}$and set $F=u^{+}+\mathcal{D}_{\varphi}\left(u^{-}\right)$.

If follows from Theorem 3.1 and formula (3.21) that we have, for $\lambda \in \mathbb{D}$

$$
v^{+}(\lambda) F(\lambda)=\frac{1}{\pi} \iint_{\mathbb{D}} \frac{v^{+}(\xi) \bar{\partial} F(\xi)}{\lambda-\xi} \mathrm{d} m(\xi)+\frac{1}{2 \mathrm{i} \pi} \int_{\mathbb{T}} \frac{u^{+}(\xi) v^{-}(\xi)}{\lambda-\xi} \mathrm{d} \xi .
$$

Also it follows from Proposition 2.5 that we have, for some $\varepsilon>0$, as $|\lambda| \rightarrow 1^{-}$

$$
\bar{\partial} F(\lambda)=\mathrm{o}\left(L_{\sigma}^{-\varepsilon}(|\lambda|)\right) .
$$

Since $u^{+} v^{-} \in L^{1}(\mathbb{T})$, the function

$$
\theta: \lambda \rightarrow \frac{1}{2 \mathrm{i} \pi} \int_{\mathbb{T}} \frac{u^{+}(\xi) v^{-}(\xi)}{\lambda-\xi} \mathrm{d} \xi
$$

belongs to $\bigcup_{p<1} H^{p}(\mathbb{D}) \subset \mathcal{N}(\mathbb{D})$. Since $u^{+} \in H^{2}(\mathbb{D})$ we see that there exists $\psi \in H^{\infty}(\mathbb{D})$, with $\|\psi\|_{H^{\infty}(\mathbb{D})}=1$, such that $\psi F \in L^{\infty}(\mathbb{D})$ and $\psi \theta \in H^{\infty}(\mathbb{D})$, so that $v^{+} \psi F \in L^{1}(\mathbb{D})$. 
Set $G=\psi F$, and set $\rho(r)=L_{\sigma}^{\varepsilon / 2}\left(r^{9}\right)$ for $r \in[0,1)$. We have $|\bar{\partial} G(\lambda)|=\mathrm{o}\left(L_{\sigma}^{-\varepsilon}(|\lambda|)\right.$ as $|\lambda| \rightarrow 1^{-}$. A fortiori, $|\bar{\partial} G(\lambda)|=\mathrm{o}\left(\rho^{-1}(|\lambda|)\right)$ as $|\lambda| \rightarrow 1^{-}$. It follows from (4.9) that $\int_{0}^{1} \log \log \rho(r) \mathrm{d} r=+\infty$, and it follows from (4.10) that $(1-r) \log \rho(r)$ is eventually increasing as $r \rightarrow 1^{-}$.

Set $A_{k}=\left\{r \in\left[1-2^{-k}, 1-2^{-k-1}\right]\left|\inf _{|\lambda|=r}\right| G(\lambda) \mid \geqslant \rho^{-1}(r)\right\}, B_{k}=\bigcup_{r \in A_{k}} r \mathbb{T}$. Since $F\left(\mathrm{e}^{\mathrm{i} t}\right) \psi\left(\mathrm{e}^{\mathrm{i} t}\right)=\lim _{r \rightarrow 1^{-}} G\left(r \mathrm{e}^{\mathrm{i} t}\right)$ does not vanish a.e. on $\mathbb{T}$ because $u \neq 0$, it follows from Lemma 4.1(i) that there exists $k_{0} \geqslant 1$ such that $\left|A_{k}\right| \geqslant 2^{-k-2}$ for $k \geqslant k_{0}$. We can also assume that $r^{9} \leqslant 1-8(1-r)$ for $1-2^{-k_{0}} \leqslant r<1$.

Let $\lambda \in \mathbb{D}$ be such that $1-2^{-k} \leqslant|\lambda| \leqslant 1-2^{-k-1}$, with $k \geqslant k_{0}$, and let $r \in A_{k+2}$. We have $2^{-k-3} \leqslant 1-r \leqslant 2^{-k-2} \leqslant r-|\lambda|$, and $r^{9} \leqslant 1-8(1-r) \leqslant 1-2^{-k} \leqslant|\lambda|$. Using Cauchy's formula, we obtain

$$
\begin{aligned}
\left|v^{+}(\lambda)\right| & =\left|\frac{1}{2 \mathrm{i} \pi} \int_{r \mathbb{T}} \frac{v^{+}(\xi)}{\xi-\lambda} \mathrm{d} \xi\right| \leqslant \frac{\rho(r)}{2 \pi(r-|\lambda|)} \int_{0}^{2 \pi}\left|v^{+}\left(r \mathrm{e}^{\mathrm{i} t}\right) G\left(r \mathrm{e}^{\mathrm{i} t}\right)\right| \mathrm{d} t \\
& \leqslant 2^{k+1} L_{\sigma}^{\varepsilon / 2}(|\lambda|) \cdot \frac{1}{\pi} \int_{0}^{2 \pi}\left|v^{+}\left(r \mathrm{e}^{\mathrm{i} t}\right) G\left(r \mathrm{e}^{\mathrm{i} t}\right)\right| \mathrm{d} t .
\end{aligned}
$$

Averaging over $A_{k+2}$, we get

$$
\begin{aligned}
\left|v^{+}(\lambda)\right| & \leqslant \frac{2^{k+1}}{\left|A_{k+2}\right|} L_{\sigma}^{\varepsilon / 2}(|\lambda|) \frac{1}{\pi} \int_{A_{k+2} \times[0,2 \pi]}\left|v^{+}\left(r \mathrm{e}^{\mathrm{i} t}\right) G\left(r \mathrm{e}^{\mathrm{i} t}\right)\right| \mathrm{d} r \mathrm{~d} t \\
& \leqslant \frac{2^{2 k+5}}{1-2^{-k}} L_{\sigma}^{\varepsilon / 2}(|\lambda|) \frac{1}{\pi} \iint_{B_{k+2}}\left|v^{+}(\xi) G(\xi)\right| \mathrm{d} m(\xi) \leqslant \frac{64}{\pi} \frac{L_{\sigma}^{\varepsilon / 2}(|\lambda|)}{(1-|\lambda|)^{2}}\left\|v^{+} G\right\|_{L^{1}(\mathbb{D})} .
\end{aligned}
$$

Since $(1-r) \log L_{\sigma}(r)$ is eventually increasing as $r \rightarrow 1^{-}$, this shows that $\left|v^{+}(\lambda)\right|=$ $\mathrm{o}\left(L_{\sigma}^{\varepsilon}(|\lambda|)\right.$ as $|\lambda| \rightarrow 1^{-}$. Hence $v^{+} \bar{\partial} F \in L^{\infty}(\mathbb{D})$, by (4.12). It follows then from (4.11) and the definition of $G$ that $v^{+} G \in L^{\infty}(\mathbb{D})$.

Using Lemma 4.1(ii), we see that

$$
\varlimsup_{r \rightarrow 1^{-}} \int_{0}^{2 \pi} \log \left|G\left(r \mathrm{e}^{\mathrm{i} t}\right)\right| \mathrm{d} t>-\infty
$$

Since $G$ is bounded on $\mathbb{D}$, this shows that

$$
\varliminf_{r \rightarrow 1^{-}} \int_{0}^{2 \pi} \log ^{+}\left|G^{-1}\left(r \mathrm{e}^{\mathrm{i} t}\right)\right| \mathrm{d} t<+\infty
$$

and so

$$
\varliminf_{r \rightarrow 1^{-}} \int_{0}^{2 \pi} \log ^{+}\left|v\left(r \mathrm{e}^{\mathrm{i} t}\right)\right| \mathrm{d} t \leqslant \varliminf_{r \rightarrow 1^{-}} \int_{0}^{2 \pi} \log ^{+}\left|G^{-1}\left(r \mathrm{e}^{\mathrm{i} t}\right)\right| \mathrm{d} t+2 \pi \log ^{+}\left[\left\|v^{+} G\right\|_{L^{\infty}(\mathbb{D})}\right]<+\infty
$$

which concludes the proof of the lemma. 
For $p \geqslant 1$ set $\theta_{p}(n)=1$ for $n \geqslant 0, \theta_{p}(n)=\mathrm{e}^{p \sqrt{|n|}}$ for $n<0$, so that $\tilde{\theta}_{p}^{*}=\tilde{\theta}_{p}=\theta_{p}$. We deduce from Lemma 4.3 the following result.

COROLlaRY 4.4. - Let $\omega \in \mathcal{S}$ be a weight satisfying the conditions of Lemma 4.3. Then every nontrivial left-invariant subspace $V$ of $\ell_{\omega^{*}}$ is contained in $\ell_{\theta_{p}^{*}}$ for some $p \geqslant 1$. Moreover $\left(V,\|\cdot\|_{\omega^{*}}\right)$ is isomorphic to $\left(V,\|\cdot\|_{\theta_{p}^{*}}\right)$, so that $\lim _{\sup } \rightarrow \infty \mathrm{e}^{-p \sqrt{n}} \cdot\left\|S_{\mid V}^{-n}\right\|<+\infty$.

Proof. - It follows from Lemma 4.3 that $\lim \sup _{|\lambda| \rightarrow 1^{-}}(1-|\lambda|) \log ^{+}\left|v^{+}(\lambda)\right|<+\infty$ for every $v \in V$. A standard application of Cauchy's inequalities shows then that

$$
\limsup _{n \rightarrow \infty}\left(\log ^{+}\left|v_{n}\right|\right) / \sqrt{n}<+\infty \quad \text { for every } v \in V .
$$

For $p \geqslant 1, q \geqslant 1$ set $V_{p, q}=\left\{v \in V\left|\sup _{n \geqslant 0}\right| v_{n} \mid \mathrm{e}^{-p \sqrt{n}} \leqslant q\|v\|_{\omega^{*}}\right\}$, so that $V_{p, q}$ is closed. We have $V=\bigcup_{p \geqslant 1, q \geqslant 1} V_{p, q}$. Hence there exists $p \geqslant 1, q \geqslant 1$ such that $\stackrel{\circ}{V}_{p, q} \neq 0$, and there exist $w \in V_{p, q}$ and $r>0$ such that

$$
\sup _{n \geqslant 0}\left|w_{n}+\frac{r v_{n}}{\|v\|_{\omega^{*}}}\right| \mathrm{e}^{-p \sqrt{n}} \leqslant q\left[r+\|w\|_{\omega^{*}}\right]
$$

for every $v \in V \backslash\{0\}$.

Set $k=q+\frac{2 q}{r}\|w\|_{\omega^{*}}$. We obtain $\left|v_{n}\right| \leqslant k \mathrm{e}^{p \sqrt{n}}\|v\|_{\omega^{*}}$ for $v \in V, n \geqslant 0$, and we have, for $v \in V$,

$$
\|v\|_{\theta_{p+1}^{*}}^{2} \leqslant \sum_{n<0}\left|v_{n}\right|^{2}+\sum_{n=0}^{\infty}\left|v_{n}\right|^{2} \mathrm{e}^{-(2 p+2) \sqrt{n}} \leqslant\left(1+k^{2} \sum_{n=0}^{\infty} \mathrm{e}^{-2 \sqrt{n}}\right)\|v\|_{\omega^{*}} .
$$

It follows from the growth conditions on $(\omega(-n))_{n \geqslant 0}$ that $\sup _{n \in \mathbb{Z}} \theta_{p+1}(n) / \omega(n)<+\infty$. Hence $\left(V,\|\cdot\|_{\omega^{*}}\right)$ is isomorphic to $\left(V,\|\cdot\|_{\theta_{p+1}^{*}}\right)$.

LEMMA 4.5. - Let $\omega \in \mathcal{S}$ be a weight satisfying the conditions of Lemma 4.3. Then for every $f \in L_{\omega}^{2}(\mathbb{T})$ there exist $g \in H^{2}$ and $k \geqslant 0$ such that

$$
\bigvee_{n \leqslant 0} z^{n} f=\bigvee_{n \leqslant-k} z^{n} g
$$

Proof. - Let $M$ be a nontrivial $z^{-1}$-invariant subspace of $L_{\omega}^{2}(\mathbb{T})$, and denote by $\pi: L_{\omega}^{2}(\mathbb{T}) \rightarrow L_{\omega}^{2}(\mathbb{T}) / M$ the canonical surjection. Using the formula $\langle\pi(f), v\rangle=\langle\hat{f}, v\rangle$ for $f \in M, v \in \widehat{M}^{\perp}$ we can identify isometrically $\left(L_{\omega}^{2}(\mathbb{T}) / M\right)^{*}$ to $\widehat{M}^{\perp}$. It follows from Corollary 4.4 that there exists $p \geqslant 1$ such that $\left(\widehat{M}^{\perp},\|\cdot\|_{\omega^{*}}\right)$ is isomorphic to $\left(\widehat{M}^{\perp},\|\cdot\|_{\theta_{p}^{*}}\right)$. Let $k>0$ such that $\|v\|_{\omega^{*}} \leqslant k\|v\|_{\theta_{p}^{*}}$ for $v \in \widehat{M}^{\perp}$, and let $f \in L_{\omega}^{2}(\mathbb{T})$. There exists $v \in \widehat{M}^{\perp}$ such that $\|v\|_{\omega^{*}}=1$ and $\|\pi(f)\|=\langle\hat{f}, v\rangle$. We obtain

$$
\|\pi(f)\|=\left|\sum_{n \in \mathbb{Z}} \hat{f}(n) v_{-n-1}\right| \leqslant\|f\|_{\theta_{p}} \cdot\|v\|_{\theta_{p}^{*}} \leqslant k\|f\|_{\theta_{p}} .
$$

Hence $\pi:\left(L_{\omega}^{2}(\mathbb{T}),\|\cdot\|_{\theta_{p}}\right) \rightarrow L_{\omega}^{2}(\mathbb{T}) / M$ is continuous. 
Set $\mathcal{B}=\left\{h \in \bar{H}^{2}\left|\sum_{n \leqslant 0}\right| \hat{h}(n) \mid \mathrm{e}^{p \sqrt{|n|}}<+\infty\right\}$. Then $\mathcal{B}$ is a Banach algebra, and $\mathrm{e}^{h} \in \mathcal{B}$ for every $h \in \mathcal{B}$. Since $\left\|z^{n} f\right\|_{\theta_{p}} \leqslant \tilde{\theta}_{p}(n)\|f\|_{\theta_{p}}=\mathrm{e}^{p \sqrt{|n|}}\|f\|_{\theta_{p}}$ for $n \leqslant 0$, we see that the series $\sum_{n \leqslant 0} \hat{h}(n) z^{n} f$ is absolutely convergent in $\ell_{\theta_{p}}$ for $f \in \ell_{\theta_{p}}, h \in \mathcal{B}$.

Computing Fourier coefficients, we see that $\sum_{n \leqslant 0} \hat{h}(n) z^{n} f=h f$. Now extend $\pi$ by continuity to $L_{\theta_{p}}^{2}(\mathbb{T})$. We obtain

$$
\pi(h f)=\sum_{n \leqslant 0} \hat{h}(n) \pi\left(z^{n} f\right) \quad\left(f \in L_{\theta_{p}}^{2}(\mathbb{T}), h \in \mathcal{B}\right) .
$$

Now let $f \in L_{\omega}^{2}(\mathbb{T})$. It follows from Corollary 4.2 that there exist $k \geqslant 0, g \in H^{2}$ and $h \in \mathcal{B}$ such that $f=z^{-k} \mathrm{e}^{h} g$. Set $h_{1}=\mathrm{e}^{h}, h_{2}=\mathrm{e}^{-h}$. We have

$$
\pi(f)=\sum_{n \leqslant 0} \hat{h}_{1}(n) \pi\left(z^{n-k} g\right), \quad \pi\left(z^{-k} g\right)=\sum_{n \leqslant 0} \hat{h}_{2}(n) \pi\left(z^{n} f\right) .
$$

The lemma follows then from (4.14).

According to [31] we will say that a left-invariant subspace $M$ of $\ell_{\omega}$ is analytic if $M^{+}:=M \cap \ell_{\omega}^{+} \neq\{0\}$. An elementary theory developed in [31, §3] shows that if $\omega \in S$ satisfies $\omega(n)=1$ for $n \geqslant 0$, and if $(\log \omega(-n)) / \sqrt{n} \rightarrow_{n \rightarrow \infty} \infty$, then every analytic left-invariant subspace $M$ of $\ell_{\omega}$ has the form $\bigvee_{n \leqslant 0} S^{n} M^{+}$. Of course, $\check{M}^{+}=\left\{f \in H^{2} \mid \hat{f} \in M^{+}\right\}$has the division property introduced in Section 2, and it follows from the results of [31] that the map $G \rightarrow \bigvee_{n \leqslant 0} S^{n} \widehat{G}$ is then a bijection from the lattice of nonzero closed subspaces of $H^{2}$ having the division property onto the lattice of analytic left-invariant subspaces of $\ell_{\omega}$.

Denote by $\mathcal{N}^{+}(\mathbb{D})$ the Smirnov class, i.e. the class of analytic functions in the disc of the form $V F$, where $V$ is inner and $F \in \mathcal{N}(\mathbb{D})$ is outer. We obtain the following result.

THEOREM 4.6. - Let $\omega \in \mathcal{S}$ be a weight satisfying the following conditions:

(1) $\omega(n)=1(n \geqslant 0)$;

(2) $\sum_{n<0} \frac{\log \omega(n)}{n^{2}}=+\infty$;

(3) $(\log \omega(-n) / \sqrt{n})_{n \geqslant 1}$ is eventually increasing;

(4) $\left(\omega(-n) / n^{\alpha}\right)_{n \geqslant 1}$ is eventually log-concave for some $\alpha>3 / 2$.

Then every $z^{-1}$-invariant subspace $F$ of $L_{\omega}^{2}(\mathbb{T})$ has the form $F=\bigvee_{n \leqslant-k} z^{n} G$ where $k \geqslant 0$ and where $G$ is a closed subspace of $H^{2}$ having the division property. In particular for every nontrivial translation invariant subspace $M$ of $\ell_{\omega}$ there exists a unique singular inner function $U$ such that $M=\bigvee_{n \in \mathbb{Z}} S^{n} \widehat{U}$ and for every nontrivial invariant subspace $N$ of $\ell_{\omega^{*}}$ there exists a unique singular inner function $U$ such that

$$
N=\left\{v \in \ell_{\omega^{*}} \mid v^{+} U \in \mathcal{N}^{+}(\mathbb{D}), \lim _{r \rightarrow 1^{-}} v^{+}(r \xi)+v^{-}(r \xi)=0 \text { a.e. on } \mathbb{T}\right\} .
$$

Proof. - It follows from Lemma 4.5 that there exists for every nontrivial $z^{-1}$-invariant subspace $F$ of $L_{\omega}^{2}(\mathbb{T})$ a nonnegative integer $k$ such that $z^{k} F \cap H^{2} \neq\{0\}$ and the first assertion follows from [31, Th. 4.5].

Now if $F$ is translation invariant then $F \cap H^{2} \neq\{0\}$ and it follows from [25, Th. 3.8] that there exists a unique singular inner function $U$ such that $F=\bigvee_{n \in \mathbb{Z}} z^{n} U$, so that $\widehat{F}=\bigvee_{n \in \mathbb{Z}} S^{n} \widehat{U}$ (this follows also from [31, Th. 4.5]). Now let $N$ be a nontrivial translation invariant subspace of $\ell_{\omega^{*}}$. There exists a unique singular inner function $U$ such that $N=\left\{v \in \ell_{\omega^{*}} \mid \widehat{U} * v=0\right\}$. The convolution product $\widehat{U} * v$ makes sense for $\sum_{n<0}\left|v_{n}\right|^{2}<+\infty, \varlimsup_{n \rightarrow \infty}\left|v_{n}\right|^{1 / n} \leqslant 1$, and the 
convolution equation $\widehat{U} * v=0$ was discussed in [25, Proposition 3.12]: we have $\widehat{U} * v=0$ iff $U . v^{+} \in \mathcal{N}^{+}(\mathbb{D})$ and $\lim _{r \rightarrow \infty} v^{+}(r \xi)+\lim _{r \rightarrow 1^{-}} v^{-}\left(r^{-1} \xi\right)=0$ a.e. on $\mathbb{T}$, which concludes the proof of the theorem.

Let $F$ be a $z^{-1}$ invariant subspace of $L_{\omega}^{2}(\mathbb{T})$. For a given $k \geqslant 0$ there exists a unique closed subspace $G$ of $H^{2}$ having the division property such that $F=\bigvee_{n \leqslant-k} z^{n} G$ but the pairs $(k, G)$ satisfying the above condition are not unique in general. The set of all these pairs is discussed in [31, Corollary 3.6].

For $\varphi \in \mathcal{N}(\mathbb{D}), \varphi \neq 0$ denote by $D(\varphi)$ the G.C.D. of all inner functions $U$ such that $U \varphi \in \mathcal{N}^{+}(\mathbb{D})$. Then $D(\varphi)$ is singular and we have as well know $\varphi=D(\varphi)^{-1} V F$ where $V$ is inner, $F$ outer and where the G.C.D. of $V$ and $D(\varphi)$ equals 1 . In particular, $\varphi D(\varphi) \in \mathcal{N}^{+}(\mathbb{D})$.

We now deduce from Theorem 4.6 a complete description of the pairs $(u, v)$, with $u \in \ell_{\omega} \backslash\{0\}$, $v \in \ell_{\omega^{*}} \backslash\{0\}$ satisfying $u * v=0$.

COROLlaRY 4.7. - Let $\omega \in \mathcal{S}$ be a weight satisfying the conditions of Theorem 4.6 and let $u \in \ell_{\omega} \backslash\{0\}, v \in \ell_{\omega^{*}} \backslash\{0\}$. Then $u * v=0$ if and only if $u$ and $v$ satisfy the two following conditions:

(1) $v^{+} \in \mathcal{N}(\mathbb{D})$, and $\lim _{r \rightarrow 1^{-}} v^{+}(r \xi)+\lim _{r \rightarrow 1^{-}} v^{-}\left(r^{-1} \xi\right)=0$ a.e. on $\mathbb{T}$;

(2) $u \in \bigvee_{n \in \mathbb{Z}} S^{n} \widehat{D\left(v^{+}\right)}$.

Proof. - Assume that $u * v=0$. Then $\bigvee_{n \in \mathbb{Z}} V S^{n} v$ is a nontrivial invariant subspace of $\ell_{\omega^{*}}$, and so $v$ satisfies (1). We also know that $\left[\bigvee_{n \in \mathbb{Z}} V S^{n} v\right]^{\perp}=\bigvee_{n \in \mathbb{Z}} V S^{n} \widehat{U}$ for some singular inner function $U$. Since $\widehat{U} * v=0, U v^{+} \in \mathcal{N}^{+}(\mathbb{D})$ and so $D(\varphi)$ is a divisor of $U$.

Hence $U \in D\left(v^{+}\right) H^{2} \in \bigvee_{n \in \mathbb{Z}} z^{n} D\left(v^{+}\right)$and so $u \in \bigvee_{n \in \mathbb{Z}} S^{n} \widehat{D\left(v^{+}\right)}$.

Conversely if $u$ and $v$ satisfy (1) and (2) then $\widehat{D\left(v^{+}\right)} * v=0$, since $D\left(v^{+}\right) v^{+} \in \mathcal{N}^{+}(\mathbb{D})$, and so $u * v=0$.

Notice that if $\omega \in \mathcal{S}$ satisfies the conditions of Theorem 4.6 then we can assume, by modifying if necessary $\omega$ on a finite set, that $\omega$ is nonincreasing. In this case it is easy to see that the map $f \rightarrow z f$ is an absolutely continuous contraction, and so inner functions are bounded multipliers on $L_{\omega}^{2}(\mathbb{T})$, see [25]. Condition (2) of Corollary 4.7 means that $u$ is the Fourier sequence of some function $f \in\left[U L_{\omega}^{2}(\mathbb{T})\right]^{-}$. We do not know any more concrete characterization of such functions.

Notice also that the translation invariant subspaces of $\ell_{\omega^{*}}$ are independent of the choice of $\omega$ if $\omega$ satisfies the hypothesis of Theorem 4.6, an illustration of the rigidity of quasianalytic structures.

Now let $r \in(0,1)$ and set $\Omega=\{z \in \mathbb{C}|r<| z \mid<1\}$. The Hardy space $H^{2}(\Omega)$ can be viewed as an "analytic analogue" of the spaces $L_{\omega}^{2}(\mathbb{T})$ considered in Theorem 4.6 (if we define $\hat{f}(n)$ to be the Laurent coefficient of $f$ of order $n$ for $f \in H^{2}(\Omega)$ we see that $H^{2}(\Omega)$ is isomorphic to the space $\ell_{\sigma}^{2}(\mathbb{Z})$ where $\sigma(n)=1$ for $n \geqslant 0, \sigma(n)=r^{n}$ for $\left.n<0\right)$. The $z$-biinvariant subspaces of $H^{2}(\Omega)$ were characterized long ago by Sarason in his thesis [46], and they have the form $U H^{2}(\Omega)$ where $U$ is an "inner function" on the annulus $\Omega$, i.e. a function having radial limits of constant modulus on both components of $\partial \Omega$. More recently, Hitt and Sarason [39, 47] characterized the $z^{-1}$-invariant subspaces (and, by symmetry, the $z$-invariant subspaces) of $H^{2}(\Omega)$. These subspaces have the form $\bigvee_{n \leqslant-k} z^{n} G$ where $G$ is a closed subspace of $H^{2}$ "nearly invariant for the backward shift", which means that $z^{-1} f \in G$ for every $f \in G$ such that $f(0)=0$.

In particular, $z^{k} F \cap H^{2} \neq\{0\}$ for some $k \geqslant 0$ if $F$ is a nontrivial $z^{-1}$-invariant subspace of $H^{2}(\Omega)$, and we see that there is a large analogy between the "analytic" and the "quasianalytic" situations. The methods used here are completely different from the methods of Hitt and Sarason. 
Since the closed subspaces of $H^{2}$ having the division property form a subclass of the class of closed subspaces of $H^{2}$ nearly invariant for the backward shift we deduce immediately from [39, 47] that these subspaces have the form $F=U \varphi\left[V H^{2}\right]^{\perp}$, where $V$ is an inner function, or $V=\{0\}$, where $U$ is a singular inner function, or $U=1$, and where $\varphi$ is an outer function satisfying $\|\varphi f\|_{2}=\|f\|_{2}$ for every $f \in\left[V H^{2}\right]^{\perp}$. These subspaces form a very large class. For example $\mathbb{C} f$ has the division property for every function $f \in H^{2}$ without zeroes in $\mathbb{D}$.

\section{The general case}

In this section we obtain results analogous to the results of Section 4 for $\omega \in \mathcal{S}$ when $(\omega(-n))_{n \geqslant 1}$ satisfies suitable growth and regularity conditions.

In order to shorten the formulations of the results, it is convenient to introduce the following regularity conditions

$$
\begin{aligned}
& \left(\frac{\log \omega(-n)}{n^{\alpha}}\right)_{n \geqslant 1} \text { is eventually increasing for every } \alpha \in(0,1) \\
& \left(\frac{\log \omega(-n)}{n}(\log n)^{A}\right)_{n \geqslant 1} \text { is eventually increasing for some } A>0 .
\end{aligned}
$$

Condition 5.2 was introduced by Borichev [14]. The second assertion of the following elementary result strenghtens [14, Lemma 1] (for $\sigma \in \mathcal{S}^{+}$we define $\bar{\sigma}$ as in Section 2, and for $\omega \in \mathcal{S}$ we define $\tilde{\omega}$ as in Section 3).

Proposition 5.1. - Let $\omega \in \mathcal{S}$, and assume that $(\omega(-n))_{n \geqslant 1}$ is eventually log-concave.

(i) If $\omega$ satisfies (5.1), then

$$
\limsup _{n \rightarrow \infty} \frac{\log \tilde{\omega}(-n)}{\log \omega(-n)}=\max \left(1, \limsup _{n \rightarrow \infty} \frac{\log \bar{\omega}_{+}(n)}{\log \omega(-n)}\right) .
$$

(ii) If $\omega$ satisfies (5.2) then $L_{\sigma}\left(r^{s}\right) L_{\sigma}^{-(1-r)^{a}}(r) \rightarrow_{r \rightarrow 1^{-}} 0$ for every $a>0$ and every $s>1$, where $\sigma=\left(\omega^{*}\right)_{+}$.

Proof. - Assume that $\omega$ satisfies (5.1). Notice that both sides of (i) are not affected if we change a finite set of values of $(\omega(n))_{n \in \mathbb{Z}}$ or if we multiply a subsequence of the sequence $(\omega(n))_{n \in \mathbb{Z}}$ by some positive constant. Hence we can assume that $\omega(0)=1$, so that $\omega^{-1}(n) \leqslant \bar{\omega}_{+}(n)$ for $n \geqslant 0$, and that $(\omega(-n))_{n \geqslant 0}$ is log-concave, so that $\omega(-n)=\sup _{p \leqslant 0} \omega(-n+p) / \omega(p)$. Hence $\tilde{\omega}(-n)=\max \left(\omega(-n), \bar{\omega}_{+}(n), \delta(n)\right)$, where

$$
\delta(n)=\sup _{1 \leqslant p \leqslant n-1} \frac{\omega(p-n)}{\omega(p)} \leqslant \sup _{1 \leqslant p \leqslant n-1} \omega(p-n) \bar{\omega}_{+}(p) .
$$

In particular $\tilde{\omega}(-n) / \omega(-n) \geqslant 1$ and $\tilde{\omega}(-n) / \bar{\omega}_{+}(n) \geqslant 1$.

Hence $\liminf \operatorname{in}_{n \rightarrow \infty} \frac{\log \tilde{\omega}(-n)}{\log \omega(-n)} \geqslant 1$ and

$$
\limsup _{n \rightarrow \infty} \frac{\log \tilde{\omega}(-n)}{\log \omega(-n)} \geqslant \limsup _{n \rightarrow \infty} \frac{\log \bar{\omega}_{+}(n)}{\log \omega(-n)} .
$$

Let $c>\max \left(1, \limsup \sup _{n \rightarrow \infty} \frac{\log \bar{\omega}_{+}(n)}{\log \omega(-n)}\right)$, and let $a \in(0,1)$. We can assume that $\bar{\omega}_{+}(n) \leqslant$ $\omega(-n)^{c}$ for $n \geqslant 1$ and that the sequence $\left((\log \omega(-n)) / n^{a}\right)_{n \geqslant 1}$ is nondecreasing. For $n \geqslant 1$, 
we obtain

$$
\log \delta(n) \leqslant c \log \omega(-n) \sup _{1 \leqslant p \leqslant n-1} \frac{p^{a}+(n-p)^{a}}{n^{a}}=2^{1-a} c \log \omega(-n) .
$$

Hence

$$
\limsup _{n \rightarrow \infty} \frac{\log \tilde{\omega}(-n)}{\log \omega(-n)} \leqslant \max \left(1, \limsup _{n \rightarrow \infty} \frac{\log \bar{\omega}_{+}(n)}{\log \omega(-n)}\right),
$$

which gives (i). Now assume that $\omega$ satisfies (5.2). For $x>0$ set

$$
\varphi(x)=\sup _{n \geqslant 0}(\log \omega(-n)-n x) .
$$

Then $\varphi$ is differentiable on $(0, \infty) \backslash \Delta$ where $\Delta$ is a countable, discrete set. An elementary computation given in the proof of [14, Lemma 1] shows that there exist $c>0$ and $\delta>0$ such that $\frac{-x \varphi^{\prime}(x)}{\varphi(x)}>c x^{-1 / A}$ for $x \in(0, \delta), x \notin \Delta$. Hence $\varphi(x) . \mathrm{e}^{-A c x^{-1 / A}}$ is decreasing on $(0, \delta)$. Since $\log L_{\sigma}\left(\mathrm{e}^{-x}\right)-\varphi(x)$ is bounded on $(0, \infty)$, we see that $\omega$ satisfies (ii).

When $\inf _{n \in \mathbb{Z}} \omega(n)=0$ it is of course no longer possible to interpret elements of $\ell_{\omega}$ as Fourier sequences of functions on the unit circle. In this situation we need to consider the set $\mathcal{M}_{\omega}$ of "convolution multipliers" on $\ell_{\omega}$. Denote by $\mathcal{E}_{o}$ the set of all sequences $u=\left(u_{n}\right)_{n \in \mathbb{Z}}$ for which the set $\left\{n \in \mathbb{Z}|| u_{n} \mid \neq 0\right\}$ is finite. A sequence $w=\left(w_{n}\right)_{n \in \mathbb{Z}}$ is a convolution multiplier on $\ell_{\omega}$ if the map $R_{w}: u \rightarrow u * w$ is continuous from $\left(\mathcal{E}_{o},\|.\|_{\omega}\right)$ into $\ell_{\omega}$. Notice that $\mathcal{M}_{\omega} \subseteq \ell_{\omega}$ since $w=w * e_{o} \in \ell_{\omega}$ for $w \in \mathcal{M}_{\omega}$, where $e_{o}=\left(\delta_{o, n}\right)_{n \in \mathbb{Z}}$. In this situation $R_{w}$ extends continously to $\ell_{\omega}$ and this extension belongs to the commutant of the shift operator $S$. It is well known [47, Section 8] that conversely there exists for every element $R$ of the commutant of $S$ a unique $w=R e_{o} \in \mathcal{M}_{\omega}$ such that $R=R_{w}$.

Also

$$
R_{w} u=\lim _{n \rightarrow \infty} \frac{1}{n+1}\left(\sum_{k=0}^{n} \sum_{|p| \leqslant k} w_{p} S^{p} u\right)
$$

for $u \in \ell_{\omega}, w \in \mathcal{M}_{\omega}$ and we have

$$
R_{w} u=\sum_{p \in \mathbb{Z}} w_{p} u_{n-p} \quad\left(u \in \ell_{\omega}, w \in \mathcal{M}_{\omega}\right)
$$

According to formula (5.3), we will write $w * u$ instead of $R_{w} u$ for $u \in \ell_{\omega}, w \in \mathcal{M}_{\omega}$. Equipped with the operator norm, $\left(\mathcal{M}_{\omega}, *\right)$ is a Banach algebra. Hence we can define

$$
\mathrm{e}^{w}=\sum_{n=0}^{\infty} \frac{w^{* n}}{n !} \in \mathcal{M}_{\omega} \quad \text { for every } w \in \mathcal{M}_{\omega},
$$

denoting by $w^{* n}$ the $n$th convolution power of $w$, so that $R_{\mathrm{e} w}=\mathrm{e}^{R_{w}}$.

Notice that if $\sum_{n \in \mathbb{Z}}\left|w_{n}\right| \tilde{\omega}(n)<+\infty$, then $w=\left(w_{n}\right)_{n \in \mathbb{Z}} \in \mathcal{M}_{\omega}$, and $\mathcal{R}_{w}=\sum_{n \in \mathbb{Z}} w_{n} S^{n}$. Also $\sum_{p \in \mathbb{Z}}\left|w_{p}\right|\left|u_{n-p}\right|<+\infty$ for every $u \in \ell_{\omega}$.

The results of this section will be based on the following slight reformulation of [18, Lemma 4.2], which we state as a lemma

LEMMA 5.2. - For every $\delta>0$ there exists $B(\delta) \geqslant 1$ such that if $F \in \mathcal{C}^{1}(D)$ and $L:(0,1) \rightarrow(0, \infty)$ satisfy the following conditions for some $r_{0} \in[0,1)$ : 
(i) $\int_{0}^{1} \log \log L(r) \mathrm{d} r=+\infty$;

(ii) $(1-r)^{B(\delta)} \log L(r)$ is non-decreasing on $\left[r_{0}, 1\right)$;

(iii) $|\bar{\partial} F(z)| \leqslant L^{-1}(|z|)\left(r_{0} \leqslant|z|<1\right)$;

(iv) $|F(z)| \leqslant L\left(|z|^{s}\right)^{\delta(1-|z|)}\left(r_{0} \leqslant|z|<1\right)$ for some $s>16$;

(v) $\varlimsup_{|z| \rightarrow 1^{-}} L\left(|z|^{n}\right)|F(z)|=+\infty$ for every $n \geqslant 1$,

then there exists $k_{0} \geqslant 1$ such that

$$
\left|\left\{r \in\left[1-2^{-k}, 1-2^{-k-1}\right]\left|\inf _{|z|=r}\right| F(z) \mid \geqslant L^{-1}(r)\right\}\right| \geqslant 2^{-k-2} \quad\left(k \geqslant k_{0}\right) .
$$

Proof. - We can assume that $1-|z|^{s} \geqslant 16(1-|z|)$ and that $L^{s}(|z|) \geqslant 4$ for $|z| \geqslant r_{0}$. Since the conclusion does not involve the values of $L$ and $F$ near the origin, the result follows immediately from [18, Lemma 4.2] applied to $2 \delta$.

The following consequence of Lemma 5.2 can be proved using essentially the same method as in the proof of [18, Theorem 6.1]. A more precise result will be proved in detail in Appendix B.

Corollary 5.3. - Let $\omega \in \mathcal{S}$. Set $\omega_{(s)}(n)=\omega(n)$ for $n \geqslant 0, \omega_{(s)}(n)=\omega^{s}(n)$ for $n<0$. Assume that $(\omega(-n))_{n \geqslant 0}$ is eventually log-concave and satisfies (5.2), and that

$$
\sum_{n<0} \frac{\log \omega(n)}{n^{2}}=+\infty
$$

If $\lim \sup _{n \rightarrow \infty} \frac{\log \bar{\omega}_{+}(n)}{\log \omega(-n)}<1 / 64$, then for every $u \in \ell_{\omega}$ and every $s<1 / 4$ there exist $k \geqslant 0$, $v \in \ell_{\omega}^{+}$and a sequence $w=\left(w_{n}\right)_{n<0}$ satisfying the following conditions:

(i) $\sum_{n<0}\left|w_{n}\right| \tilde{\omega}_{(s)}(n)<+\infty$;

(ii) $S^{k} u=\mathrm{e}^{w} * v$.

Before using Lemma 5.2 and Corollary 5.3 to obtain our main theorem we need to recall some more elementary results from [31]. Let $\sigma \in \mathcal{S}^{+}$. Set

$$
K_{\sigma}(r)=\sum_{n=0}^{\infty} \bar{\sigma}(n+1) r^{n}, \quad M_{\sigma}(r)=\left[\sum_{n=0}^{\infty} \sigma^{-2}(n) r^{2 n}\right]^{1 / 2}
$$

for $r \in[0,1)$, where $\bar{\sigma}(n)=\sup _{p \geqslant 0} \sigma(p) / \sigma(n+p)$ for $n \geqslant 0$. For $p \geqslant 1$ denote by $\mathcal{A}_{p, \sigma}$ the set of functions $f \in \mathcal{H}(\mathbb{D})$ which can be written as a quotient $f=g / h$ where $g \in \mathcal{H}(\mathbb{D})$, $h \in \mathcal{H}(\mathbb{D})$ satisfy the conditions $|g(\lambda)| \leqslant K_{\sigma}(|\lambda|),|h(\lambda)| \leqslant M_{\sigma}(|\lambda|)(\lambda \in \mathbb{D}),|h(0)| \geqslant 1 / p$. Define $\mathcal{B}_{p, \sigma}$ in a similar way by replacing the condition $|g(\lambda)| \leqslant K_{\sigma}(|\lambda|)$ by the weaker condition $|g(\lambda)| \leqslant 2 K_{\sigma}(|\lambda|) M_{\sigma}(|\lambda|)$. For $r \in[0,1)$ set

$$
\Lambda_{\sigma}(r)=\sup \left\{|f(\lambda)|, f \in \mathcal{A}_{p, \sigma},|\lambda|=r\right\}, \quad \Delta_{\sigma}(r)=\sup \left\{|f(\lambda)|, f \in \mathcal{B}_{p, \sigma},|\lambda|=r\right\} .
$$

Finally set, for $p \geqslant 1$

$$
\sigma^{(p)}(n)=\inf _{0<r<1} r^{-n} \Lambda_{\sigma}(r), \quad \sigma^{[p]}(n)=\inf _{0<r<1} r^{-n} \Delta_{\sigma}(r) .
$$

The sequences $\left(\omega_{+}^{(p)}(n)\right)_{n \geqslant 0}$ and $\left(\omega_{+}^{[p]}(n)\right)_{n \geqslant 0}$ play for general weights $\omega \in \mathcal{S}$ the role played by the sequences $\left(\mathrm{e}^{p \sqrt{n}}\right)_{n \geqslant 0}$ when $\omega(n)=1$ for $n \geqslant 0$. For example if $M$ is a nontrivial left-invariant subspace of $\ell_{\omega}$ such that $M \cap \ell_{\omega}^{+} \neq\{0\}$ then there exists $p \geqslant 1$ such that 
$\sup _{n \geqslant 0} \frac{\left|v_{n}\right|}{\omega_{+}^{(p)}(n)}<+\infty$ for every $v \in M^{\perp}$ and such that $\limsup _{n \rightarrow \infty} \frac{\left\|S_{\mid M^{\perp}}^{-n}\right\|}{\omega_{+}^{[p]}(n)}<+\infty$, where $M^{\perp}=\left\{v \in \ell_{\omega^{*}} \mid\langle u, v\rangle=0(u \in M)\right\}$, see [31, Section 3].

We will say that a closed subspace $N$ of $\ell_{\omega}^{+}$has the division property if $\check{N}=\left\{f \in H_{\omega_{+}}\right.$| $\hat{f} \in N\}$ has the division property in the sense of Definition 2.1.

If

$$
\sum_{n=0}^{\infty} \frac{\left[\omega_{+}^{(p)}(n)\right]^{2}}{\omega^{2}(-n)}<+\infty
$$

for every $p \geqslant 1$, it follows from [31, Corollary 3.5] that $N=\left(\bigvee_{n \leqslant 0} S^{n} N\right) \cap \ell_{\omega}^{+}$and $\ell_{\omega}=\ell_{\omega}^{+}+\bigvee_{n \leqslant 0} S^{n} N$ for every nontrivial closed subspace $N$ of $\ell_{\omega}^{+}$having the division property. Also, $M=\bigvee_{n \leqslant 0} S^{n}\left(M \cap \ell_{\omega}^{+}\right)$for every left-invariant subspace $M$ of $\ell_{\omega}$ such that $M \cap \ell_{\omega}^{+} \neq\{0\}$.

Precise evaluations in various concrete cases of the growth of the weights $\sigma^{(p)}$ and $\sigma^{[p]}$, based on the Matsaev-Mogulskii estimates of quotient of analytic functions [42], are given in [31, Section 4]. We state as a lemma the following consequence of these results.

Lemma 5.4. - Let $\omega \in \mathcal{S}$. Assume that $(\omega(-n))_{n \geqslant 1}$ is eventually log-concave, and that $\left(\frac{\log \omega(-n)}{n^{\alpha}}\right)_{n \geqslant 1}$ is eventually increasing for every $\alpha \in[0,1)$.

If $\lim \sup _{n \rightarrow \infty} \frac{\log \bar{\omega}_{+}(n)}{\log \omega(-n)}<1$, then

$$
\limsup _{n \rightarrow \infty} \frac{\log \omega_{+}^{[p]}(n)}{\log \omega(-n)}=\limsup _{n \rightarrow \infty} \frac{\log \bar{\omega}_{+}(n)}{\log \omega(-n)}
$$

for every $p \geqslant 1$, and $M=\bigvee_{n \leqslant 0} S^{n}\left(M \cap \ell_{\omega}^{+}\right)$for every left-invariant subspace $M$ of $\ell_{\omega}$ such that $M \cap \ell_{\omega}^{+} \neq\{0\}$. Also $N=\left(\bigvee_{n \leqslant 0} S^{n} N\right) \cap \ell_{\omega}^{+}$and $\ell_{\omega}=\ell_{\omega}^{+}+\bigvee_{n \leqslant 0} S^{n} N$ for every nontrivial closed subspace $N$ of $\ell_{\omega}^{+}$having the division property.

Proof. - Clearly, $\omega_{+}^{[p]}(n) \geqslant \bar{\omega}_{+}(n+1) \geqslant[\tilde{\omega}(1)]^{-1} \bar{\omega}_{+}(n)$ for $n \geqslant 0$, and so

$$
\limsup _{n \rightarrow \infty} \frac{\log \omega_{+}^{[p]}(n)}{\log \omega(-n)} \geqslant \limsup _{n \rightarrow \infty} \frac{\log \bar{\omega}_{+}(n)}{\log \omega(-n)} \quad \text { for } p \geqslant 1 .
$$

Set $\sigma(n)=\omega^{-1}(n)$ for $n \geqslant 0$, and let $c \in\left(\lim \sup _{n \rightarrow \infty} \frac{\log \bar{\omega}_{+}(n)}{\log \omega(-n)}, 1\right)$. Then $\bar{\omega}_{+}(n) \leqslant \sigma^{c}(n)$ when $n$ is sufficiently large. Since $\omega_{+}^{-1}(n) \leqslant \omega(0)^{-1} \bar{\omega}_{+}(n)$ for $n \geqslant 0$, we see that there exists an integer $k \geqslant 1$ such that $M_{\omega_{+}}(r) \leqslant k M_{\sigma^{c}}(r)$ and $K_{\omega_{+}}(r) \leqslant k K_{\sigma^{c}}(r)$ for $r \in[0,1)$. Hence $\mathcal{B}_{\omega_{+, p}} \subset \mathcal{B}_{\sigma^{c}, p k^{2}}$, and $\omega_{+}^{[p]} \leqslant\left[\sigma^{c}\right]^{\left[p k^{2}\right]}$ for $p \geqslant 1$.

It follows from [31, Proposition 4.4] that $\frac{\log \left(\sigma^{c}\right)^{[q]}(n)}{\log \sigma^{-c}(n)} \rightarrow_{n \rightarrow \infty} 1$ for every $q \geqslant 1$. Hence $\limsup _{n \rightarrow \infty} \frac{\log \omega_{+}^{[p]}(n)}{\log \omega(-n)} \leqslant c$ for every $p \geqslant 1$, which proves the first assertion.

It follows from the growth condition on $\omega$ that $\sum_{n<0} \omega^{-\varepsilon}(n)<+\infty$ for every $\varepsilon>0$, and the other assertions follow then from [31, Corollary 3.5].

Before proceeding to the proof of the main result of the paper, we need the following technical observation

Lemma 5.5. - Let $\omega \in \mathcal{S}$. Assume that $\omega$ satisfies the following conditions:

(i) $(\omega(-n))_{n \geqslant 1}$ is eventually log-concave; 
(ii) $\left(\frac{\log \omega(-n)}{n}(\log n)^{A}\right)_{n \geqslant 1}$ is eventually increasing for some $A>0$;

(iii) $\limsup _{n \rightarrow \infty} \frac{\log \bar{\omega}_{+}(n)}{\log \omega(-n)}<1 / 200$.

Then

$$
\limsup _{r \rightarrow 1} \frac{\log M_{\omega_{+}}(r)}{(1-r) \log L_{\left(\omega^{*}\right)_{+}}\left(r^{200}\right)}<+\infty,
$$

and $K_{\omega_{+}}(r)=\mathrm{O}\left(L_{\left(\omega^{*}\right)_{+}}\left(r^{200}\right)\right)$ as $r \rightarrow 1^{-}$.

Proof. - Set $\sigma=\left(\omega^{*}\right)_{+}$, so that $\sigma(n)=\omega(-n-1)$ for $n \geqslant 0$. We have

$$
K_{\omega_{+}}(r)=\sum_{n=0}^{\infty} \bar{\omega}_{+}(n+1) r^{n} \quad \text { and } \quad M_{\omega_{+}}(r)=\left(\sum_{n=0}^{\infty} \omega^{-2}(n) r^{2 n}\right)^{1 / 2}
$$

for $r \in[0,1)$. Since $\bar{\omega}_{+}(n+1) \geqslant[\tilde{\omega}(1)]^{-1} \omega(0) \omega^{-1}(n)$ for $n \geqslant 0, M_{\omega_{+}}(r) \leqslant \frac{\tilde{\omega}(1)}{\omega(0)} K_{\omega_{+}}(r)$, and it suffices to show that

$$
\limsup _{r \rightarrow 1^{-}} \frac{\log K_{\omega_{+}}(r)}{(1-r) \log L_{\sigma}\left(r^{200}\right)}<+\infty .
$$

Set $\Delta(r)=\sup _{n \geqslant 0} \bar{\omega}_{+}(n+1) r^{n}$, so that $K_{\omega_{+}}(r) \leqslant\left(1-r^{\beta}\right)^{-1} \Delta\left(r^{1-\beta}\right)$ for $\beta \in(0,1)$.

We have

$$
\limsup _{n \rightarrow \infty} \frac{\log \bar{\omega}_{+}(n+1)}{\log \sigma^{-1}(n)}=\limsup _{n \rightarrow \infty} \frac{\log \bar{\omega}_{+}(n)}{\log \omega(-n)}<1 / 200 .
$$

Choose $d<1 / 200$ such that $\bar{\omega}_{+}(n+1) \leqslant \sigma^{-d}(n)$ when $n$ is sufficiently large, and choose $\beta \in(0,1)$ such that $\frac{1-\beta}{d}>200$. It follows in particular from Proposition 5.1(ii) that

$$
(1-r)^{k} \log L_{\sigma}(r) \underset{r \rightarrow 1^{-}}{\rightarrow} \infty \quad \text { for every } k>0 .
$$

Then $\limsup \sup _{r \rightarrow 1^{-}} \Delta(r) L_{\sigma}^{-d}\left(r^{\frac{1}{d}}\right) \leqslant 1$, and so $\lim \sup _{r \rightarrow 1^{-}} K_{\omega_{+}}(r) L_{\sigma}^{-d-1}\left(r^{\frac{1-\beta}{d}}\right)=0$. The lemma follows then immediately from Proposition 5.1(ii).

We will use the following lemma to circumvent the fact that the sequence $\left(w_{n}\right)_{n<0}$ provided by Corollary 5.3 is not a convolution multiplier on $\ell_{\omega}$.

LEMma 5.6. - Let $\omega \in \mathcal{S}$. Assume that $\omega$ satisfies the following growth and regularity conditions:

(1) $\sum_{n<0} \frac{\log \omega(n)}{n^{2}}=+\infty$;

(2) $\left(\frac{\log \omega(-n)}{n^{\alpha}}\right)_{n \geqslant 1}$ is eventually increasing for every $\alpha \in(0,1)$;

(3) $\left(\omega(-n) / n^{\alpha}\right)_{n \geqslant 1}$ is eventually log-concave for some $\alpha>3 / 2$;

(4) $\lim \sup _{r \rightarrow 1^{-}} \frac{\log M_{\omega_{+}}(r)}{(1-r) \log L_{\left(\omega^{*}\right)_{+}}\left(r^{200}\right)}<+\infty$;

(5) $K_{\omega_{+}}(r)=\mathrm{O}\left(L_{\left(\omega^{*}\right)_{+}}\left(r^{13}\right)\right)$ as $r \rightarrow 1^{-}$.

Then there exists $s>4$ such that $\lim \sup _{|\lambda| \rightarrow 1^{-}} L_{\left(\omega_{*}\right)}^{-1}\left(|\lambda|^{s}\right)\left|v^{+}(\lambda)\right|<+\infty$ for every $v \in \ell_{\omega^{*}}$ which is not left-cyclic.

Proof. - We will use the same strategy as in the proof of Lemma 4.3. Set $\sigma=\left(\omega^{*}\right)_{+}$, $K=K_{\omega_{+}}, M=M_{\omega_{+}}$. Let $u \in \ell_{\omega}, v \in \ell_{\omega^{*}}$. For $n \geqslant 0$ we have

$$
\left|\sum_{p<0} u_{n-p} v_{p}\right| \leqslant\|u\|_{\omega}\|v\|_{\omega^{*}} \sup _{p<0} \frac{1}{\omega^{*}(p) \omega(n-p)}=\|u\|_{\omega}\|v\|_{\omega^{*}} \bar{\omega}(n+1) .
$$


We obtain

$$
\left|P^{+}\left(u^{+} v^{-}\right)(\lambda)\right| \leqslant\|u\|_{\omega}\|v\|_{\omega^{*}} K(|\lambda|) \quad(\lambda \in \mathbb{D}) .
$$

Now assume that $v \in \ell_{\omega^{*}}$ is not left-cyclic, and let $u \in \ell_{\omega} \backslash\{0\}$ such that $v \perp \bigvee_{n<0} S^{n} u$. With the notations of Section 2, let $\varphi \in W(\sigma)$ and set $F=u^{+}+\mathcal{D}_{\varphi}\left(u^{-}\right), G=\mathcal{C}^{+}\left(v^{+} \partial \bar{\partial} F\right)$, so that $G \in L^{1}(\mathbb{D}), H=-P^{+}\left(u^{+} v^{-}\right)$. It follows from Theorem 3.1 that $v^{+} F=G+H$.

Assume that there exists a continuous increasing function $\rho$ on $[0,1)$ and $k_{0} \geqslant 1$ satisfying

$$
\left|\left\{r \in\left[1-2^{-k}, 1-2^{-k-1}\right]\left|\inf _{|\lambda|=r}\right| F(\lambda) \mid \geqslant \rho^{-1}(r)\right\}\right| \geqslant 2^{-k-2} \quad\left(k \geqslant k_{0}\right) .
$$

Since $3 / 8-1 / 32>1 / 3$, we can assume that $1-r^{1 / 3}<(3 / 8-1 / 32)(1-r)$ for $r \in\left[1-2^{-k_{0}}, 1\right]$. Set

$$
A_{k}=\left\{r \in\left[1-2^{k}+2^{-k-5}, 1-2^{-k-1}-2^{-k-2}+2^{-k-4}\right]\left|\inf _{|\lambda|=r}\right| F(\lambda) \mid \geqslant \rho^{-1}(r)\right\},
$$

$B_{k}=\bigcup_{r \in A_{k}} r \mathbb{T}$. It follows from (5.6) that $\left|A_{k}\right| \geqslant 2^{-k-5}$ for $k \geqslant k_{0}$.

Let $\lambda \in \mathbb{D}$ such that $1-|\lambda| \leqslant 2^{-k_{0}}$, and let $k \geqslant k_{0}$ such that $1-2^{-k} \leqslant|\lambda|<1-2^{-k-1}$. We have $\frac{1}{\left|A_{k+1}\right|} \leqslant 2^{k+6} \leqslant \frac{64}{1-|\lambda|}$. Also

$$
1-|\lambda|^{1 / 3}<\left(\frac{3}{8}-\frac{1}{32}\right)(1-|\lambda|) \leqslant 3.2^{-k-3}-2^{-k-5}=2^{-k-2}+2^{-k-3}-2^{-k-5},
$$

and so $r<|\lambda|^{1 / 3}$ and $\frac{1}{r-|\lambda|} \leqslant 2^{k+6}=\frac{64}{1-|\lambda|}$ for $r \in A_{k+1}$. Let $r \in A_{k+1}$. We have

$$
\begin{aligned}
\left|v^{+}(\lambda)\right| & =\frac{1}{2 \pi}\left|\int_{r \mathbb{T}} \frac{v^{+}(\xi) \mathrm{d} \xi}{\xi-\lambda}\right| \leqslant \frac{32}{\pi(1-|\lambda|)} \int_{0}^{2 \pi} r\left|v^{+}\left(r \mathrm{e}^{\mathrm{i} t}\right)\right| \mathrm{d} t \\
& \leqslant \frac{32 \rho(r)}{\pi(1-|\lambda|)} \int_{0}^{2 \pi}\left[r\left|G\left(r \mathrm{e}^{\mathrm{i} t}\right)\right|+\left|H\left(r \mathrm{e}^{\mathrm{i} t}\right)\right|\right] \mathrm{d} t \\
& \leqslant \frac{32 \rho\left(|\lambda|^{1 / 3}\right)}{\pi(1-|\lambda|)} \int_{0}^{2 \pi} r\left|G\left(r \mathrm{e}^{\mathrm{i} t}\right)\right| \mathrm{d} t+\frac{64 \rho\left(|\lambda|^{1 / 3}\right)}{1-|\lambda|} K\left(|\lambda|^{1 / 3}\right)\|u\|_{\omega}\|v\|_{\omega^{*}} .
\end{aligned}
$$

Averaging over $A_{k+1}$, we obtain

$$
\left|v^{+}(\lambda)\right| \leqslant \frac{32 \rho\left(|\lambda|^{1 / 3}\right)}{\pi(1-|\lambda|)\left|A_{k+1}\right|} \int_{B_{k+1}}|G(\xi)| \mathrm{d} m(\xi)+\frac{64}{1-|\lambda|} \rho\left(|\lambda|^{1 / 3}\right) K\left(|\lambda|^{1 / 3}\right)\|u\|_{\omega}\|v\|_{\omega^{*}}
$$

We have

$$
\iint_{B_{k+1}}|G(\xi)| \mathrm{d} m(\xi) \leqslant \iint_{\mathbb{D}}|G(\xi)| \mathrm{d} m(\xi)<+\infty
$$

and

$$
\frac{1}{\left|A_{k+1}\right|} \leqslant \frac{64}{1-|\lambda|} \leqslant 64 K(|\lambda|) \leqslant 64 K\left(|\lambda|^{1 / 3}\right)
$$


Hence we have

$$
\left|v^{+}(\lambda)\right|=\mathrm{O}\left(\frac{\rho\left(|\lambda|^{1 / 3}\right) K\left(|\lambda|^{1 / 3}\right)}{1-|\lambda|}\right) \quad\left(|\lambda| \rightarrow 1^{-}\right)
$$

Since the sequence $\left(\frac{\log \omega(-n)}{n^{\alpha}}\right)_{n \geqslant 1}$ is eventually increasing for every $\alpha \in(0,1)$, an elementary verification shows that $\left(\frac{\log \sigma^{-1}(n)}{n^{\alpha}}\right)_{n \geqslant 1}$ is also eventually increasing for every $\alpha \in(0,1)$. It follows then from [51] that $(1-r)^{k} \log L_{\sigma}(r)$ is eventually increasing as $r \rightarrow 1^{-}$for every $k \geqslant 1$, and we obtain

$$
\frac{\log L_{\sigma}\left(r^{s}\right)}{\log L_{\sigma}(r)} \underset{r \rightarrow 1^{-}}{\rightarrow} 0 \quad(s>1)
$$

Now choose $a \in(12,12.5)$, so that $16 a<200$. Set $\rho(r)=L_{\sigma}\left(r^{a}\right)=\left(L_{\sigma^{1 / a}}(r)\right)^{a}$ for $r \in(0,1)$. It follows again from [43] that $(1-r)^{k} \log \rho(r)$ is eventually increasing as $r \rightarrow 1^{-}$. Since $\sum_{n \geqslant 1} \frac{\log \sigma(n)}{n^{2}}=+\infty, \int_{0}^{1} \log \log \rho(r)=+\infty$.

It follows from Proposition 2.5 that we can choose $\varphi \in W(\sigma)$ such that

$$
|\bar{\partial} F(\lambda)|=\left|\bar{\partial} \mathcal{D}_{\varphi}\left(u^{-}\right)(\lambda)\right|=\mathrm{O}\left(L_{\sigma}^{-\varepsilon}(|\lambda|)\right)
$$

for some $\varepsilon>0$ as $|\lambda| \rightarrow 1^{-}$. It follows then from (5.8) that $|\bar{\partial} F(\lambda)|=\mathrm{o}\left(\rho^{-1}(|\lambda|)\right)$ as $|\lambda| \rightarrow 1^{-}$. Also $|F(\lambda)|=\mathrm{O}(M(|\lambda|))$ as $|\lambda| \rightarrow 1^{-}$. Let

$$
\delta>\limsup _{r \rightarrow 1^{-}}(1-r) \frac{\log M(r)}{(1-r) \log L_{\sigma}\left(r^{200}\right)} .
$$

Then there exists $r_{0} \in(0,1)$ such that

$$
M(r) \leqslant L_{\sigma}\left(r^{200}\right)^{\delta(1-r)}=\rho\left(r^{\frac{200}{a}}\right)^{\delta(1-r)}
$$

for $r \in\left(r_{0}, 1\right)$. Since $F=u^{+}+\mathcal{C}^{+}(\bar{\partial} F)$ we cannot have $|F(\lambda)| \rightarrow|\lambda| \rightarrow 1^{-} 0$ because otherwise the function $-u^{-}$would provide an analytic extension of $u^{+}$to $\mathbb{C}$ vanishing at infinity, hence vanishing identically.

Since $\frac{200}{a}>16$, it follows then from Lemma 5.2 that $F$ and $\rho$ satisfy (5.6). Obviously, $\frac{L_{\sigma}(r)}{1-r} \rightarrow_{r \rightarrow 1^{-}} \infty$. Since $K(r)=\mathrm{O}\left(L_{\sigma}\left(r^{13}\right)\right)$ as $r \rightarrow 1^{-}$, and since $a<13$, we deduce from (5.7) that $\left|v^{+}(\lambda)\right|=\mathrm{O}\left(L_{\sigma}^{3}\left(r^{a / 3}\right)\right)$. Hence $\left|v^{+}(\lambda)\right|=\mathrm{O}\left(L_{\sigma}\left(r^{b}\right)\right)$ for every $b \in(4, a / 3)$.

We set as before $\omega_{(s)}(n)=\omega(n)(n \geqslant 0), \omega_{(s)}(n)=\omega^{s}(n)(n<0)$ for $\omega \in \mathcal{S}, s>0$.

COROLlaRY 5.7. - If $\omega \in \mathcal{S}$ satisfies the conditions of Lemma 5.6, then there exists $s<1 / 4$ such that $\sum_{n=0}^{\infty}\left|v_{n}\right|^{2} \omega^{-2 s}(-n-1)<+\infty$ for every $v \in \ell_{\omega^{*}}$ which is not left-cyclic. Moreover the norms $\|\cdot\|_{\omega_{(s)}^{*}}$ and $\|\cdot\|_{\omega^{*}}$ are equivalent on every nontrivial left-invariant subspace of $\ell_{\omega^{*}}$.

Proof. - Set again $\sigma=\left(\omega^{*}\right)_{+}$. There exists $s_{0} \in(0,1 / 4)$ such that $\left|v^{+}(\lambda)\right|=\mathrm{O}\left(L_{\sigma}\left(|\lambda|^{1 / s_{0}}\right)\right)$ as $|\lambda| \rightarrow 1^{-}$for every $v \in \ell_{\omega^{*}}$ which is not left-cyclic.

Let $s_{1} \in\left(s_{0}, 1 / 4\right)$ and $s \in\left(s_{1}, 1 / 4\right)$. It follows from (5.8) that

$$
L_{\sigma}\left(|\lambda|^{1 / s_{0}}\right) \leqslant L_{\sigma}^{s_{1}}\left(|\lambda|^{1 / s_{1}}\right)=L_{\sigma^{s_{1}}}(|\lambda|)
$$

when $1-|\lambda|$ is sufficiently small, and it follows then from Cauchy's inequalities that

$$
\limsup _{n \rightarrow \infty}\left|v_{n}\right| \omega^{-s_{1}}(-n-1)=\limsup _{n \rightarrow \infty}\left|v_{n}\right| \sigma^{s_{1}}(n)<+\infty
$$


We obtain

$$
\sum_{n=0}^{\infty}\left|v_{n}\right|^{2} \omega^{-2 s}(-n-1) \leqslant\left(\sup _{n \geqslant 0}\left|v_{n}\right| \omega^{-s_{1}}(-n-1)\right)^{2} \sum_{n=0}^{\infty} \omega^{2 s_{1}-2 s}(-n-1)<+\infty .
$$

Now let $V$ be a nontrivial left-invariant subspace of $\ell_{\omega^{*}}$. Then $V \subset \ell_{\omega_{(s)}^{*}}$.

The injection $\ell_{\omega_{(s)}^{*}} \rightarrow \ell_{\omega^{*}}$ is clearly continuous. Using the topology of coordinatewise convergence, we see that the graph of the injection $\left(V,\|\cdot\|_{\omega^{*}}\right) \rightarrow\left(\ell_{\omega_{(s)}^{*}},\|\cdot\|_{\omega_{(s)}^{*}}\right)$ is closed. Hence $\left(V,\|\cdot\|_{\omega^{*}}\right)$ is isomorphic to $\left(V,\|\cdot\|_{\omega_{(s)}^{*}}\right)$.

Let $\sigma \in \mathcal{S}^{+}$, and let $N$ be a $z$-invariant subspace of the weighted Hardy space $H_{\sigma}=H_{\sigma}^{2}(\mathbb{D})$. We define the (possibly infinite) index of $N$ by the formula $\operatorname{Ind}(N)=\operatorname{dim}(N / z N)$ ). Also we will say that $N$ is zero-free if $\bigcap_{f \in N} f^{-1}(\{0\})=\emptyset$. It is easy to see, and well-known also (see for example [31]), that $N$ has the division property introduced in Section 2 if and only if $N$ is zero-free and $\operatorname{Ind}(N)=1$. The following theorem is the main result of the paper (according to the notations introduced in Section 2 we set $\bar{\omega}_{+}(n)=\sup _{p \geqslant 0} \frac{\omega(p)}{\omega(n+p)}$ for $\left.\omega \in \mathcal{S}\right)$.

THEOREM 5.8. - Let $\omega \in \mathcal{S}$ be a weight satisfying the following conditions:

(1) $\sum_{n<0} \frac{\log \omega(n)}{n^{2}}=+\infty$;

(2) $\left(\frac{\log \omega(-n)}{n}(\log n)^{A}\right)_{n \geqslant 1}$ is eventually increasing for some $A>0$;

(3) $\left(\omega(-n) / n^{\alpha}\right)_{n \geqslant 1}$ is eventually log-concave for some $\alpha>3 / 2$;

(4) $\limsup _{n \rightarrow \infty} \frac{\log \bar{\omega}_{+}(n)}{\log \omega(-n)}<1 / 200$.

Then for every $u \in \ell_{\omega}$ there exists $v \in \ell_{\omega}^{+}$and $k \geqslant 0$ such that $\bigvee_{n \leqslant 0} S^{n} u=\bigvee_{n \leqslant-k} S^{n} v$, and for every nontrivial left-invariant subspace $M$ of $\ell_{\omega}$ there exists $k \geqslant 0$ and a closed subspace $N$ of $H_{\omega}^{+}$having the division property such that $M=\bigvee_{n \leqslant-k} S^{n} \widehat{N}$. In particular, every nontrivial translation invariant subspace $M$ of $\ell_{\omega}$ has the form $M=\bigvee_{n \leqslant 0} S^{n} \widehat{N}$ where $N$ is a zero-free $z$-invariant subspace of $H_{\omega_{+}}$of index 1.

Proof. - We could use the same method as in the proof of Corollary 4.5, but we will use here a duality argument. Denote by $W$ the set of elements of $\ell_{\omega^{*}}$ which are not left-cyclic. It follows from Lemma 5.5 that $\omega$ satisfies the conditions of Lemma 5.6, and it follows from Corollary 5.7 that $W \subset \ell_{\omega_{(s)}^{*}}$ for some $s>1 / 4$.

Let $u \in \ell_{\omega} \backslash\{0\}$. It follows from Corollary 5.3 that there exists $\varphi \in \mathcal{M}_{\omega_{(s)}}, \omega \in \ell_{\omega}^{+}$and $k \geqslant 0$ such that $S^{k} u=\mathrm{e}^{\varphi} * w$, with $\varphi_{n}=0$ for $n \geqslant 0$ and $\sum_{n<0}\left|\varphi_{n}\right| \tilde{\omega}_{(s)}(n)<+\infty$.

Set $\psi=\mathrm{e}^{\varphi}$, so that $\psi \in \mathcal{M}_{\omega_{(s)}}, \psi_{n}=0$ for $n>0$ and $\sum_{n \leqslant 0}\left|\psi_{n}\right| \tilde{\omega}_{(s)}(n)<+\infty$. We have $\left\|S^{k} u-\sum_{n=-p}^{0} \psi_{n} S^{n} w\right\|_{\omega_{(s)}} \rightarrow p \rightarrow \infty 0$. Now let $v \in \ell_{\omega^{*}}$ and assume that $\left\langle S^{n} w, v\right\rangle=0$ for $n \leqslant 0$. Then $v \in W \subset \ell_{\omega_{(s)}^{*}}$ and so

$$
\left\langle S^{k} u, v\right\rangle=\lim _{p \rightarrow \infty} \sum_{n=-p}^{0} \psi_{n}\left\langle S^{n} w, v\right\rangle=0
$$

This shows that $S^{k} u \in \bigvee_{n \leqslant 0} S^{n} w$, and the same argument shows that $w \in \bigvee_{n \leqslant 0} S^{n+k} u$. Hence $\bigvee_{n \leqslant 0} S^{n} u=\bigvee_{n \leqslant-k} S^{n} w$.

The other assertions follow then immediately from Lemma 5.4. 
For $\sigma \in \mathcal{S}^{+}, p \geqslant 1$ define again $\sigma^{(p)}$ and $\sigma^{[p]}$ by (5.4), so that $\log \sigma^{[p]}(n)=\mathrm{O}(\sqrt{n})$ as $n \rightarrow \infty$ for every $p \geqslant 1$ if $\sum_{n=1}^{\infty} \frac{\log \bar{\sigma}_{+}(n)}{n^{3 / 2}}<+\infty$ (see [31, Section 4]). Since

$$
\lim _{n \rightarrow \infty} \frac{\sigma^{(p)}(n+k)}{\sigma^{(p)}(n)}=\lim _{n \rightarrow \infty} \frac{\sigma^{[p]}(n+k)}{\sigma^{[p]}(n)}=1
$$

for every $k \geqslant 1$, we obtain, using the observations of [31] mentioned before Lemma 5.4.

COROLlary 5.9. - Let $\omega$ be a weight satisfying the conditions of Theorem 5.8. Then for every $v \in \ell_{\omega^{*}}$ which is not left-cyclic there exists $p \geqslant 1$ such that $\lim _{\sup } \operatorname{su}_{n \rightarrow \infty} \frac{\left|v_{n}\right|}{\omega_{+}^{(p)}(n)}<+\infty$, and for every nontrivial left-invariant subspace $V$ of $\ell_{\omega^{*}}$ there exists $p \geqslant 1$ such that $\lim \sup _{n \rightarrow \infty} \frac{\left\|S_{\mid V}^{-n}\right\|}{\omega_{+}^{[p]}(n)}<+\infty$. In particular,

$$
\limsup _{n \rightarrow \infty} \frac{\log \left\|S_{\mid V}^{-n}\right\|}{\sqrt{n}}<+\infty
$$

for every nontrivial left-invariant subspace $V$ of $\ell_{\omega^{*}}$ if $\sum_{n=1}^{\infty} \frac{\log \bar{\omega}_{+}(n)}{n^{3 / 2}}<+\infty$.

We thus see that Lemma 5.6 and Corollary 5.7 were only a step in the proof of Theorem 5.8, since Corollary 5.9 gives a much better result.

When $\sigma(n)=(n+1)^{-1 / 2}$, the weighted Hardy space $H_{\sigma}=H_{\sigma}^{2}(\mathbb{D})$ is the usual Bergman space $B^{2}(\mathbb{D})$ of square integrable holomorphic functions in the disc [36]. According to Korenblum [41] we will say that a function $U \in B^{2}(\mathbb{D})$ is Bergman-inner if $\|U\|_{B^{2}(\mathbb{D})}=1$ and if $\left\langle U, z^{n} U\right\rangle=0$ for $n \geqslant 1$. A Bergman-inner function will be said to be singular if it has no zeroes in $\mathbb{D}$.

It follows from the Aleman-Richter-Sundberg theorem [3] that the zero-free $z$-invariant subspaces of $B^{2}(\mathbb{D})$ of index 1 are the subspaces of the form $\bigvee_{n \geqslant 0} z^{n} U$ where $U$ is a singular Bergman-inner function [31, Proposition 5.1]. We obtain in particular the following result.

Corollary 5.10. - Set $\omega(n)=(n+1)^{-1 / 2}(n \geqslant 0), \omega(n)=\mathrm{e}^{|n| /(\log |n|+1)^{a}}(n<0)$, where $0<a \leqslant 1$. Then every nontrivial translation invariant subspace of $\ell_{\omega}$ has the form $\bigvee_{n \in \mathbb{Z}} S^{n} \widehat{U}$ where $U$ is a singular Bergman-inner function.

If $\omega$ is as above, then every nontrivial left-invariant subspace of $\ell_{\omega^{*}}$ is (isomorphically) contained in $\ell_{\omega_{p}^{*}}$ where $\omega_{p}(n)=(n+1)^{-1 / 2}$ for $n \geqslant 0, \omega_{p}(n)=\mathrm{e}^{p \sqrt{|n|}}$ for $n<0$. We do not know a concrete description of these subspaces.

Using conditions analogous to conditions (4) and (5) of Lemma 5.5 it is possible to obtain formulations of Theorem 5.8 by replacing the condition $\left(\frac{\log \omega(-n)}{n}(\log n)^{A}\right)_{n \geqslant 1}$ eventually increasing for some $A>0$ by the condition $\left(\frac{\log \omega(-n)}{n^{\delta}}\right)_{n \geqslant 1}$ eventually increasing for some suitable $\delta \in(1 / 2,1)$.

But the constants involved in these conditions would depend on $\delta$ (the constant $B(\delta)$ is not given explicitely in Lemma 4.2 of [18]) and on the real $\alpha>3 / 2$ for which $\left(\omega(-n) / n^{\alpha}\right)_{n \geqslant 1}$ is eventually log-concave, and there are minor technical complications to obtain a version of Corollary 5.3 suitable for this purpose. We leave the details to the reader. Notice that the results of this section apply to the weights $\omega^{(c)}$ defined by the formula $\omega^{(c)}(n)=\mathrm{e}^{-n / \log (n+1)}$ for $n \geqslant 0$, $\omega^{(c)}(-n)=\mathrm{e}^{c(|n| / \log (|n|+1))}$ for $n<0$ if $c>200$. The constant 200 is indeed not best possible. It would be interesting to see whether Theorem 5.8 holds for $\omega^{(c)}$ for $c>1$, but significant progress in this direction would necessitate to revisit the proof of [18, Lemma 4.2]. We will not do it here. 


\section{New counterexamples in the Fréchet case, and an open problem}

If $\mathcal{F}$ is a space of sequences on $\mathbb{Z}$ we will set as above $\mathcal{F}^{+}=\left\{u=\left(u_{n}\right)_{n \in \mathbb{Z}} \in \mathcal{F} \mid u_{n}=0\right.$ $(n<0)\}$ and $\mathcal{F}^{-}=\left\{u=\left(u_{n}\right)_{n \in \mathbb{Z}} \in \mathcal{F} \mid u_{n}=0(n \geqslant 0)\right\}$.

Set

$$
\mathcal{U}=\left\{u=\left.\left(u_{n}\right)_{n \in \mathbb{Z}}\left|\limsup _{n \rightarrow-\infty}\right| u_{n}\right|^{1 /|n|}<1, \limsup _{n \rightarrow \infty}\left|u_{n}\right|^{1 / n} \leqslant 1\right\} .
$$

For $r \in[0,1)$ set $\Omega_{r}=\{\lambda \in \mathbb{C}|r<| \lambda \mid<1\}$ and denote by $\hat{f}(n)$ the $n$th Laurent coefficient of $f \in \mathcal{H}\left(\Omega_{r}\right)$. Equip the inductive limit $\mathcal{V}=\lim _{\rightarrow} \mathcal{H}\left(\Omega_{r}\right)$ with the usual locally convex topology. Then $\widehat{\mathcal{V}}=\mathcal{U}$, and $\mathcal{U}$, equipped with the topology induced by the topology of $\mathcal{V}$, is a topological algebra with respect to convolution. Using the formula $\langle u, v\rangle=\sum_{n \in \mathbb{Z}} u_{n} v_{-n-1}$ we can identify $\mathcal{U}^{*}$ to $\mathcal{U}$ and, since $(\mathcal{U}, *)$ is an integral domain, $\mathcal{U}$ does not have any nontrivial translation invariant subspace (the right and left-invariant subspaces of $\mathcal{U}$ are described in [26]).

Notice that $\mathcal{U}=\mathcal{U}^{+} \oplus \mathcal{U}^{-}$is the direct sum of a nuclear Fréchet space and a D.F.N. space (i.e., the strong dual of a nuclear Fréchet space).

More sophisticated counterexamples to the translation invariant subspace problem for reflexive, locally convex complete linear spaces of sequences can be found in the literature. Set

$$
\mathcal{A}=\bigcap_{p \geqslant 1}\left\{u=\left.\left(u_{n}\right)_{n \in \mathbb{Z}}\left|\sum_{n<0}\right| u_{n}\right|^{2} \mathrm{e}^{2|n|-\frac{2|n|}{p}}<+\infty, \limsup _{n \rightarrow \infty}\left|u_{n}\right|^{1 / n} \leqslant 1\right\}
$$

and for $\beta \in(1 / 2,1)$ set

$$
\mathcal{A}_{\beta}=\bigcap_{p \geqslant 1}\left\{u=\left.\left(u_{n}\right)_{n \in \mathbb{Z}}\left|\sum_{n<0}\right| u_{n}\right|^{2} \mathrm{e}^{p|n|^{\beta}}<+\infty, \limsup _{n \rightarrow \infty}\left|u_{n}\right|^{1 / n} \leqslant 1\right\} .
$$

Also set

$$
\begin{aligned}
& \mathcal{B}^{+}=\bigcup_{p \geqslant 1}\left\{u=\left.\left(u_{n}\right)_{n \geqslant 0}\left|\sum_{n=0}^{\infty}\right| u_{n}\right|^{2} \mathrm{e}^{\frac{-p n}{\log (n+2)}}<+\infty\right\}, \\
& \mathcal{B}^{-}=\bigcap_{p \geqslant 1}\left\{u=\left.\left(u_{n}\right)_{n<0}\left|\sum_{n<0}\right| u_{n}\right|^{2} \mathrm{e}^{\frac{p|n|}{\log (n \mid+1)}}<+\infty\right\}
\end{aligned}
$$

and $\mathcal{B}=\mathcal{B}^{+} \oplus \mathcal{B}^{-}$, so that $\mathcal{B}^{+}$is a D.F.N. space, $\mathcal{B}^{-}$a nuclear Fréchet space, and $\mathcal{B}=\mathcal{B}^{*}$ a reflexive, locally convex complete topological algebra with respect to convolution.

Atzmon [6,7] showed that the Fréchet spaces $\mathcal{A}$ and $\mathcal{A}_{\beta}$ have no nontrivial translation invariant subspaces, and Borichev [14], using the theory of asymptotically holomorphic functions, showed that a similar result holds for $\mathcal{B}$ (which means that $\mathcal{B}$ has no nontrivial closed ideals). In all these examples, the spectrum of the shift operator is empty. We now use the results of Section 5 to produce examples of Fréchet spaces of sequences on $\mathbb{Z}$ having no nontrivial translation invariant subspaces for which the spectrum of the shift operator equals the unit circle.

THEOREM 6.1.- Let

$$
\mathcal{D}=\bigcap_{p \geqslant 1}\left\{u=\left.\left(u_{n}\right)_{n \in \mathbb{Z}}\left|\sum_{n<0}\right| u_{n}\right|^{2} \mathrm{e}^{\frac{p|n|}{\log (|n|+1)}}<+\infty, \sum_{n=0}^{\infty}\left|u_{n}\right|^{2} \mathrm{e}^{\frac{-2 n}{\sqrt{\log (n+2)}}}<+\infty\right\}
$$


and for $\beta \in(1 / 2,1)$ set

$$
\mathcal{E}_{\beta}=\bigcap_{p \geqslant 1}\left\{u=\left.\left(u_{n}\right)_{n \in \mathbb{Z}}\left|\sum_{n<0}\right| u_{n}\right|^{2} \mathrm{e}^{p|n|^{\beta}}<+\infty, \sum_{n=0}^{\infty}\left|u_{n}\right|^{2} \mathrm{e}^{\frac{-2 n}{\log (n+2)}}<+\infty\right\} .
$$

Then the spaces $\mathcal{D}$ and $\mathcal{E}_{\beta}$ do not possess any nontrivial translation invariant subspace.

Proof. - Set $\omega_{p}(n)=\mathrm{e}^{p|n| / \log (|n|+1)}$ for $n<0, \omega_{p}(n)=\mathrm{e}^{-n / \sqrt{\log (n+2)}}$ for $n \geqslant 0$, so that $\mathcal{D}=\bigcap_{p \geqslant 1} \ell_{\omega_{p}}$. We can identify $\mathcal{D}^{*}$ to $\bigcup_{p \geqslant 1} \ell_{\omega_{p}^{*}} \subset \mathcal{B}$. Let $u \in \mathcal{D}$ and assume that there exists $v \in \mathcal{D}^{*} \backslash\{0\}$ such that $u * v=0$. There exists $p \geqslant 1$ such that $v \in \ell_{\omega_{p}^{*}}$. Then $\left(\omega_{p}^{*}\right)_{+}$is eventually $\log$-convex, so that $\sup _{n \geqslant 0}\left(\left(\bar{\omega}_{p}^{*}\right)_{+}(n)\right) / \omega_{p}(-n)<+\infty$. Also $\omega_{p}^{*}$ satisfies the conditions of Theorem 5.8, and it follows then from Corollary 5.8 that $\limsup _{n \rightarrow \infty}\left|u_{n}\right| / \omega_{p}(-n)<+\infty$, so that $u^{+} \in \mathcal{B}^{+}$and $u \in \mathcal{B}$. Since $(\mathcal{B}, *)$ is an integral domain, $u=0$ and $\mathcal{D}$ does not possess any nontrivial translation invariant subspace.

Now fix $\beta \in(1 / 2,1)$ and set $\omega_{p}(n)=\mathrm{e}^{p|n|^{\beta}}$ for $n<0, \omega_{p}(n)=\mathrm{e}^{-n / \log (n+2)}$ for $n \geqslant 0$, so that $\mathcal{E}_{\beta}=\bigcap_{p \geqslant 1} \ell_{\omega_{p}}$. We can again identify $\mathcal{E}_{\beta}^{*}$ to $\bigcup_{p \geqslant 1} \ell_{\omega_{p}^{*}}$. Let $M \neq \mathcal{E}_{\beta}$ be a translation invariant subspace of $\mathcal{E}_{\beta}$, and set $N=\left\{v \in \mathcal{E}_{\beta}^{*} \mid\langle u, v\rangle=0(u \in M)\right\}$, so that $N$ is a translation invariant subspace of $\mathcal{E}_{\beta}^{*}$. Clearly, $N \neq\{0\}$, and so $N \cap \ell_{\omega_{p}^{*}} \neq\{0\}$ for some $p \geqslant 1$. Since $N \cap \ell_{\omega_{p}^{*}}$ is closed in $\ell_{\omega_{p}^{*}}$, it follows from Theorem 5.8 that $N \cap \ell_{\omega_{p}^{*}}^{+} \neq\{0\}$. Hence $N^{+} \neq\{0\}$, where $N^{+}=\left\{v=\left(v_{n}\right)_{n \in \mathbb{Z}} \in N \mid v_{n}=0(n<0)\right\}$. Set $\sigma_{p}=\left(\omega_{p}^{*}\right)_{+}$, and set $V=\left\{f \in \bigcup_{p \geqslant 1} H_{\sigma_{p}} \mid\right.$ $\hat{f} \in N\}$. Then $V$ is a zero-free $z$-invariant subspace of $\bigcup_{p \geqslant 1} H_{\sigma_{p}}$ which is closed with respect to the locally convex inductive topology on $\bigcup_{p \geqslant 1} H_{\sigma_{p}}$. But it follows from a result of MatsaevMogulskii [42] that closed $z$-invariant subspaces of $\bigcup_{p \geqslant 1} H_{\sigma_{p}}$ are determined by their zero-set, and so $V=\bigcup_{p \geqslant 1} H_{\sigma_{p}}, N^{+}=\bigcup_{p \geqslant 1} \ell_{\omega_{p}^{*}}^{+}$and $N=\mathcal{E}_{\beta}^{*}$, so that $M=\{0\}$.

We now go back to weighted Hilbert spaces of sequences on $\mathbb{Z}$. There were no specific requirements on $\omega_{+}=\left.\omega\right|_{\mathbb{Z}^{+}}$in Theorem 5.8. In fact we have the following result.

Proposition 6.2.- For every $\sigma \in \mathcal{S}^{+}$there exists $\omega \in \mathcal{S}$, satisfying the conditions of Theorem 5.8, such that $\left.\omega\right|_{\mathbb{Z}^{+}}=\sigma$.

Proof. - Define $\bar{\sigma}$ as in Section 2. For $x>0$ set $V(x)=\sum_{n=0}^{\infty} \bar{\sigma}(n)^{10^{3}} \mathrm{e}^{-n x}, W(x)=$ $\frac{\log V(x)}{x^{2}}, \varphi(x)=V(x)^{1 / x^{2}}=\mathrm{e}^{W(x)}$. It follows from Hadamard's three circles theorem that $\log V$ is convex on $(0, \infty)$. Hence $\varphi$ is decreasing, convex and infinitely differentiable on $(0, \infty)$.

Now set $\theta(y)=\inf _{x>0}(\varphi(x)+x y)$ for $y>0$ and set $\omega(n)=\sigma(n)$ for $n \geqslant 0, \omega(-1)=\mathrm{e}^{\theta(1)}$ and $\omega(n)=\mathrm{e}^{\theta(|n|)+\frac{|n|}{\log |n|}}$ for $n \leqslant-2$. It follows from standard properties of Legendre transforms that $\theta$ is increasing, concave and infinitely differentiable on $(0, \infty)$, and $\frac{\log \theta(y)}{y} \rightarrow y \rightarrow \infty 0$. Set $\rho(n)=\omega(-n-1)$ for $n \geqslant 0$. Then $\rho \in \mathcal{S}^{+}$, and since $\sigma=\left.\omega\right|_{\mathbb{Z}^{+}} \in \mathcal{S}^{+}$, an elementary verification shows that $\omega \in \mathcal{S}$. Clearly, $\left(\frac{\log \omega(-n)}{n^{\alpha}}\right)_{n \geqslant 1}$ is eventually log-concave for every $\alpha>0$, and $\sum_{n<0} \frac{\log \omega(n)}{n^{2}}=+\infty$. We have $\bar{\sigma}(n)^{10^{3}} \mathrm{e}^{-n x} \leqslant V(x)$ for $x>0$, and so

$$
10^{3} \log \bar{\sigma}(n) \leqslant \inf _{x>0}(\log V(x)+n x)=\inf _{0<x<1}(\log V(x)+n x)=\inf _{0<x<1}(\varphi(x)+n x)=\theta(n)
$$

when $n$ is sufficiently large, and $\lim \sup _{n \rightarrow \infty} \frac{\log \bar{\omega}_{+}(n)}{\log \omega(-n)} \leqslant 10^{-3}$.

Since $\log V$ is decreasing we have $2 W(x)<-x W^{\prime}(x)$ for $x>0$. Hence

$$
\left|W^{\prime}(x)\right|>\frac{2 \log V(x)}{x^{3}}>\frac{1}{x^{2}} \quad \text { and } \quad \frac{\log \left|W^{\prime}(x)\right|}{\left|W^{\prime}(x)\right|}<x^{2} \log 1 / x^{2}
$$


when $x$ is sufficiently small, so that

$$
\frac{\log \left(-W^{\prime}(x)\right)}{-x W^{\prime}(x)} \underset{x \rightarrow 0^{+}}{\rightarrow} 0
$$

Hence

$$
\limsup _{x \rightarrow 0^{+}} \frac{W(x)+\log \left(-W^{\prime}(x)\right)}{-x W^{\prime}(x)} \leqslant 1 / 2 .
$$

For every $y>0$ there exists a unique $x>0$ such that $\theta(y)=\varphi(x)+x y$, and $\varphi^{\prime}(x)=-y$. It follows from standard properties of Legendre transforms that $\varphi(x)=\sup _{u>0} \theta(u)-x u$, so that $x=\theta^{\prime}(y) \rightarrow y \rightarrow \infty 0$.

Hence

$$
\begin{aligned}
\limsup _{y \rightarrow \infty}\left[\frac{\theta(y)}{y \theta^{\prime}(y)}-1\right] \log y & =\limsup _{x \rightarrow 0^{+}} \frac{\varphi(x)}{-x \varphi^{\prime}(x)} \log \left(-\varphi^{\prime}(x)\right) \\
& =\limsup _{x \rightarrow 0^{+}} \frac{W(x)+\log \left(-W^{\prime}(x)\right)}{-x W^{\prime}(x)} \leqslant 1 / 2 .
\end{aligned}
$$

We thus see that there exists $y_{0}>0$ such that $\frac{\theta(y)}{y \theta^{\prime}(y)}-1<\frac{1}{\log y}$ for $y>y_{0}$. We obtain

$$
\frac{\theta(y)}{\theta(y)-y \theta^{\prime}(y)}>1+\log y>\log y \quad \text { for } y>y_{0},
$$

which shows that $\left(\frac{\theta(n)}{n} \log n\right)_{n \geqslant 1}$ is eventually increasing. A fortiori $\left(\frac{\log \omega(-n)}{n} \log n\right)_{n \geqslant 1}$ is eventually increasing, and $\omega$ satisfies the conditions of Theorem 5.8.

Theorem 5.8 and Proposition 6.2 suggest the following problem:

Problem 3. - Does the weighted Hardy space $H_{\sigma}=H_{\sigma}^{2}(\mathbb{D})$ possess a nontrivial, zero-free $z$-invariant subspace of index 1 for every $\sigma \in \mathcal{S}^{+}$?

Denote by $T$ the shift operator $f \rightarrow z f$ on $H_{\sigma}$. It is not difficult to see that the nontrivial zero-free $z$-invariant subspaces of index 1 of $H_{\sigma}$ are the $z$-invariant subspaces $M$ for which $\sigma\left(T_{\mid M^{\perp}}^{*}\right) \subset \mathbb{T}$ (or, equivalently, $\sigma\left(T_{M}\right) \subset \mathbb{T}$, where $T_{M}$ is the operator induced by $T$ on $H_{\sigma} / M$ ). All singly generated zero-free invariant subspace of $H_{\sigma}$ have index 1 , but the existence of such subspaces is unknown in the general case. A negative answer to Problem 6.3 would of course provide an example of a weight $\omega \in \mathcal{S}$ for which $\ell_{\omega}=\ell_{\omega}^{2}(\mathbb{Z})$ has no nontrivial translation invariant subspace.

When $\sigma \in \mathcal{S}$ is nonincreasing and when $\sigma(n) \rightarrow_{n \rightarrow \infty} 0$, the shift $T$ on $H_{\sigma}$ belongs to the class $\mathbb{A}_{\aleph_{o}}$ of Brown-Chevreau-Pearcy and it follows in particular from [20] that for every singular inner function $U$ there exists $f \in H_{\sigma}$ such that $f \notin \bigvee_{n \geqslant 0} z^{n} U f$, which shows that $z$-invariant subspaces of $H_{\sigma}$ are not determined by their sets of zeroes in $\mathbb{D}$. A recent construction of Borichev [15], based on lacunary series, shows more generally that if $\sigma \in \mathcal{S}^{+}$is $\log$-convex, and if $\inf _{n \geqslant 0} \sigma(n)=0$, then $H_{\sigma}$ possesses for every $p$ such that $2 \leqslant p \leqslant \infty$ a zerofree, $z$-invariant subspace of index $p$. The existence of nontrivial zero-free $z$-invariant subspaces of $H_{\sigma}$ seems to be an open problem for arbitrary $\sigma \in \mathcal{S}^{+}$.

When $\sigma$ is log-convex there are three available methods to obtain partial positive answers to Problem 3. The so-called Keldysh method, developped by Nikolski in [43], gives in particular explicit examples of functions $f \in H_{\sigma}$ without zeroes in $\mathbb{D}$, such that $\bigvee_{n \geqslant 0} z^{n} f \subsetneq H_{\sigma}$ when $\sigma(n)=\mathrm{e}^{-n^{\alpha}}, 1 / 2 \leqslant \alpha<1$. Another approach, based on functions of "extremal rate of growth 
and decrease" was proposed by Hedenmalm and the second author in an unpublished paper [37], and developped in the case where $\sigma(n)=\mathrm{e}^{-\sqrt{n}}$. A variant of this method was recently used by Borichev, Hedenmalm and the second author [17] to produce zero-free $z$-invariant subspaces of arbitrary index in all "large" Bergman spaces. Atzmon [8,9] obtained recently new positive results on the existence of translation invariant subspaces of $\ell_{\omega}$, based on the theory of entire functions of zero exponential type. It follows from Atzmon's results that if $\sigma \in \mathcal{S}^{+}$is log-convex, and if $\sup _{n \geqslant 0}\left[\sigma(n+1) \sigma(n-1) / \sigma(n)^{2}\right]^{1 / n}<+\infty$, then $H_{\sigma}$ possesses a nontrivial $z$-invariant subspace $M$ such that $\sigma\left(T_{\mid M^{\perp}}^{*}\right)=\{1\}$, which gives a positive answer to Problem 3 in this situation. We refer to [31, Section 5] for a detailed discussion of these results. It seems that the answer to Problem 3 ought to be positive for log-convex weights, but the general case remains unclear.

\section{Appendix A. Strong convexity properties}

In this appendix we give a proof of Proposition 2.5. This result is a discrete version of results from [16, Appendix B]. Our direct approach, based on the inversion formula for Laplace transforms, seems somewhat simpler.

We denote as above by $L_{\sigma}$ the Legendre transform of $\sigma \in \mathcal{S}^{+}$, see Definition 2.4.

Let $\sigma \in \mathcal{S}^{+}$. For $\alpha \in \mathbb{R}$, we define $\sigma_{\alpha} \in \mathcal{S}^{+}$by the formula

$$
\sigma_{\alpha}(n)=(n+1)^{\alpha} \sigma(n) \quad(n \geqslant 0) .
$$

Clearly, $\sigma_{\mu}$ is log-convex for $\mu<\alpha$ if $\sigma_{\alpha}$ is log-convex.

LEMmA A.1. - Let $\sigma \in \mathcal{S}^{+}$, and assume that $\sigma_{\alpha}$ is eventually log-convex for some $\alpha>0$. Then

$$
\varlimsup_{r \rightarrow 1^{-}} L_{\sigma}^{1-\mu / \alpha}(r) L_{\sigma_{\mu}}^{-1}(r)<+\infty \quad(0 \leqslant \mu<\alpha) .
$$

Proof. - We can assume that $\sigma_{\alpha}$ is log-convex, so that $\sigma$ is log-convex. Set $r_{0}=0, r_{n}=\frac{\sigma(n)}{\sigma(n-1)}$ for $n \geqslant 1$. We have $r_{n}=\frac{\sigma_{\alpha}(n)}{\sigma_{\alpha}(n-1)}\left(1+\frac{1}{n}\right)^{-\alpha}$ for $n \geqslant 1$. Since $\sigma_{\alpha}$ is log-convex,

$$
r_{n}^{n}=\left[\frac{\sigma_{\alpha}(n)}{\sigma_{\alpha}(n-1)}\right]^{n}\left(1+\frac{1}{n}\right)^{-n \alpha} \geqslant \frac{\sigma(n)}{\sigma(0)}(n+1)^{\alpha}\left(1+\frac{1}{n}\right)^{-n \alpha} .
$$

Hence there exists $c>0$ satisfying

$$
r_{n}^{n} \geqslant c \sigma(n)(n+1)^{\alpha} \quad(n \geqslant 1) .
$$

Now let $\mu \in(0, \alpha)$, and set $p=\frac{\alpha}{\alpha-\mu}$, so that $\alpha(p-1)-p \mu=0$. Let $r \geqslant r_{1}$ and let $n \geqslant 1$ be such that $r \in\left[r_{n}, r_{n+1}\right]$. We have

$$
L_{\sigma_{\mu}}^{p}(r) \geqslant(n+1)^{-p \mu} \sigma^{-p}(n) r^{n p} \geqslant(n+1)^{-p \mu} \sigma^{-p}(n) r_{n}^{n(p-1)} r^{n} .
$$

Using (2.20) and (A.2) we obtain

$$
L_{\sigma_{\mu}}^{p}(r) \geqslant c^{p-1} \sigma^{-1}(n) r^{n}=c^{p-1} L_{\sigma}(r) \quad\left(r \geqslant r_{1}\right)
$$

and the lemma follows. 
LEMMA A.2. - Let $\sigma \in \mathcal{S}^{+}$, and assume that $\sigma_{\alpha}$ is eventually log-convex for some $\alpha>1 / 2$. Then $W(\sigma)$ contains a strictly decreasing function $\phi$ which is continuously differentiable on $[0,1)$ and satisfies

$$
\limsup _{r \rightarrow 1^{-}} \phi(r) L_{\sigma_{1 / 2}}(r)<+\infty .
$$

Proof. - For $\phi \in L_{+}^{2}(0,1)$ set $\tilde{\phi}(t)=\mathrm{e}^{-t} \phi^{2}\left(\mathrm{e}^{-t}\right)(t>0)$. Then $\tilde{\phi} \in L^{1}\left(\mathbb{R}^{+}\right)$and we have

$$
\sigma_{\varphi}^{2}(n)=2 \mathcal{L}(\tilde{\varphi})(2 n+1) \quad(n \geqslant 0),
$$

where we denote by $\mathcal{L}(\tilde{\phi}): z \rightarrow \int_{0}^{\infty} \mathrm{e}^{-z t} \tilde{\phi}(t) \mathrm{d} t$ the usual Laplace transform of $\tilde{\phi}$.

Denote by $\Gamma$ the gamma-function and set, for $s>0$

$$
e_{s}(t)=\frac{t^{s-1} \mathrm{e}^{-t}}{\Gamma(s)} \quad(t>0)
$$

Then $\mathcal{L}\left(e_{s}\right)(z)=(z+1)^{-s}$ for $\operatorname{Re} z \geqslant 0$.

Now assume that $\sigma_{\alpha}$ is eventually log-convex for some $\alpha>1 / 2$. Replacing $\alpha$ by $\alpha-\varepsilon$ is necessary, we can assume that $\sigma_{\alpha}(n) \rightarrow_{n \rightarrow \infty} 0$. Then $W\left(\sigma_{\alpha}\right)$ contains a function $F$ which is continuous on $[0,1)$.

Set $G=\widetilde{F}, \psi(t)=\mathrm{e}^{t}\left(G * e_{2 \alpha}\right)(t)$ for $t>0$ and $\varphi(r)=[\psi(-\log r)]^{1 / 2}$ for $r \in(0,1)$. Since $\widetilde{\varphi}=G * e_{2 \alpha} \in L^{1}\left(\mathbb{R}^{+}\right), \varphi \in L^{2}(0,1)$. Also $G * e_{2 \alpha}=\left(G * e_{2 \alpha-1}\right) * e_{1}$ and so $\psi(x)=\int_{0}^{x}\left(G * e_{2 \alpha-1}\right)(t) \mathrm{e}^{t} \mathrm{~d} t$ for $x>0$. Since $G$ and $e_{2 \alpha-1}$ are continuous on $(0, \infty)$, $G * e_{2 \alpha-1}$ is continuous on $(0, \infty)$ and we see that $\varphi$ is strictly decreasing and continuously differentiable on $(0,1)$.

We have

$$
\sigma_{\varphi}^{2}(n)=2 \mathcal{L}\left(G * e_{2 \alpha}\right)(2 n+1)=\frac{\sigma_{F}^{2}(n)}{(2 n+2)^{2 \alpha}},
$$

and so $\varphi \in W(\sigma)$. By using the inversion formula for Fourier transforms we obtain, for $t>0$

$$
\begin{aligned}
\left(G * e_{2 \alpha}\right)(t) \mathrm{e}^{-(2 n+1) t} & =\frac{1}{2 \pi}\left|\int_{-\infty}^{\infty} \frac{\mathcal{L}(G)(2 n+1+\mathrm{i} y)}{(2 n+2+\mathrm{i} y)^{2 \alpha}} \mathrm{e}^{\mathrm{i} y t} \mathrm{~d} y\right| \leqslant \frac{\sigma_{F}^{2}(n)}{4 \pi} \int_{-\infty}^{\infty} \frac{\mathrm{d} y}{|2 n+2+\mathrm{i} y|^{2 \alpha}} \\
& =\frac{\sigma_{F}^{2}(n)}{(2 n+2)^{2 \alpha-1}} \frac{1}{4 \pi} \int_{-\infty}^{\infty} \frac{\mathrm{d} s}{\left(1+s^{2}\right)^{\alpha}}=(n+1) \sigma_{\varphi}^{2}(n) \frac{1}{2 \pi} \int_{-\infty}^{\infty} \frac{\mathrm{d} s}{\left(1+s^{2}\right)^{\alpha}} .
\end{aligned}
$$

Hence

$$
\varphi^{2}(r) \leqslant\left[\frac{2}{\pi} \int_{-\infty}^{\infty} \frac{\mathrm{d} s}{\left(1+s^{2}\right)^{\alpha}}\right](n+1) \sigma_{\varphi}^{2}(n) r^{-2 n}
$$

for $n \geqslant 0,1 / 2 \leqslant r<1$. Since $\varphi \in W(\sigma), \varphi(r)=\mathrm{O}\left(L_{\sigma_{1 / 2}}^{-1}(r)\right)$ as $r \rightarrow 1^{-}$. Modyfing $\varphi$ near 0 if necessary, we can arrange $\varphi$ to be continuously differentiable on $[0,1)$.

Proof of Proposition 2.5. - Choose $\phi \in L_{+}^{2}(0,1)$ satisfying the conditions of Lemma A.2 with respect to $\sigma$. Then $\phi$ satisfies (i) and $\phi(r)=\mathrm{O}\left(L_{\sigma_{1 / 2}}^{-1}(r)\right)$ as $r \rightarrow 1^{-}$. Also it follows from Lemma A.1 that $L_{\sigma_{1 / 2}}^{-1}(r)=\mathrm{O}\left(L_{\sigma}^{1 / 2 \alpha-1}(r)\right)$ as $r \rightarrow 1^{-}$. We obtain

$$
\phi^{2}(r)=\mathrm{O}\left(L_{\sigma}^{1 / \alpha-2}(r)\right) \quad \text { as } r \rightarrow 1^{-} .
$$


Let $\delta \in(0,1-3 / 2 \alpha)$ and set $\beta=\alpha(1-\delta)-1$, so that $\beta>1 / 2$. We have

$$
\left[\sum_{n=0}^{\infty} \sigma^{-2}(n) r^{2 n}\right]^{1 / 2} \leqslant\left(\sum_{n=0}^{\infty}(n+1)^{-2 \beta}\right)^{1 / 2} L_{\sigma_{-\beta}}(r)
$$

for $0 \leqslant r<1$.

Using again Lemma A.1 we see that $L_{\sigma_{-\beta}}^{1-\frac{\beta}{\alpha+\beta}}(r)=\mathrm{O}\left(L_{\sigma}(r)\right)$ as $r \rightarrow 1^{-}$. We obtain

$$
\left(\sum_{n=0}^{\infty} \sigma^{-2}(n) r^{2 n}\right)^{1 / 2}=\mathrm{O}\left(L_{\sigma}^{1+\beta / \alpha}(r)\right) \quad \text { as } r \rightarrow 1^{-} .
$$

Since $\delta=1-\frac{\beta+1}{\alpha}$, this gives

$$
\phi^{2}(r)\left(\sum_{n=0}^{\infty} \sigma^{-2}(n) r^{2 n}\right)^{1 / 2}=\mathrm{O}\left(L_{\sigma}^{-\delta}(r)\right) \quad \text { as } r \rightarrow 1^{-}
$$

and assertion (ii) of Proposition 2.5 follows immediately from Proposition 2.3.

\section{Appendix B. Factorization theorems}

We give here quantitative versions of known factorization theorems [18, Theorems 6.1 and 6.3]. These results, which have some interest in themselves, are more precise than the factorization results needed in Sections 4 and 5.

Recall that if $\omega \in \mathcal{S}$ and if $w=\left(w_{n}\right)_{n \in \mathbb{Z}}$ satisfies $\sum_{n \in \mathbb{Z}}\left|w_{n}\right| \tilde{\omega}(n)<+\infty$, then $w$ is a convolution multiplier on $\ell_{\omega}$, and $w * u=\sum_{n \in \mathbb{Z}} w_{n} S^{n} u$ for $u \in \ell_{\omega}$.

We first give an easy elementary result.

Proposition B.1. - Let $\omega \in \mathcal{S}$ and let $u \in \ell_{\omega}$. If $\lim \sup _{n \rightarrow \infty}\left|u_{-n}\right|^{1 / n}<1$ then there exists $k \geqslant 0, v \in \ell_{\omega}^{+}$and $w=\left(w_{n}\right)_{n<0}$ such that $\limsup _{n \rightarrow-\infty}\left|w_{n}\right|^{1 /|n|}<1$ and $S^{k} u=\mathrm{e}^{w} * v$.

In particular

$$
\bigvee_{n \leqslant 0} S^{n} u=\bigvee_{n \leqslant-k} S^{n} v
$$

Proof. - Set $\Omega_{r}=\{\lambda \in \mathbb{C}|r<| \lambda \mid<1\}$ for $r \in[0,1)$, set $\rho=\limsup _{n \rightarrow \infty}\left|u_{-n}\right|^{1 / n}$ and set $f(\lambda)=\sum_{n \in \mathbb{Z}} u_{n} \lambda^{n}$ for $\lambda \in \Omega_{\rho}$. Let $r \in(\rho, 1)$. By using a suitable Weierstrass product we can write $f=B g$ where $B \in \mathcal{H}(\mathbb{D})$ and where $g \in \mathcal{H}\left(\Omega_{r}\right)$ has no zeroes in $\Omega_{r}$. In this situation we have as well-known $g=z^{p} \mathrm{e}^{h}$, where $h \in \mathcal{H}\left(\Omega_{r}\right)$ and where $p \in \mathbb{Z}$ is the winding number of $g(s \mathbb{T})$ with respect to the origin for $s \in(r, 1)$. Writing $h=h_{1}+h_{2}$, with $h_{1} \in \mathcal{H}(\mathbb{D})$, $h_{2} \in \mathcal{H}_{0}(\mathbb{C} \backslash r \overline{\mathbb{D}})$ we obtain a factorization $f=z^{-k} f_{1} \mathrm{e}^{f_{2}}$, where $k \geqslant 0, f_{1} \in \mathcal{H}(\mathbb{D})$, $f_{2} \in \mathcal{H}_{0}(\mathbb{C} \backslash r \overline{\mathbb{D}})$, so that $\mathrm{e}^{-f_{2}} f=z^{-k} f_{1}$. Then $w=\hat{f}_{2}$ satisfies $\lim _{\sup _{n \rightarrow-\infty}}\left|w_{n}\right|^{1 /|n|}<1$, $w_{n}=0$ for $n \geqslant 0$, and $\mathrm{e}^{-w} * u=\mathrm{e}^{-R_{w}} u=S^{-k} \hat{f}_{1} \in \ell_{\omega}$, where $R_{\omega}$ is defined as in Section 5 . Set $v=\hat{f}_{1}$. Then $v \in \ell_{\omega}^{+}$, and $S^{k} u=\mathrm{e}^{w} * v$.

Now set $\varphi=\mathrm{e}^{w}, \psi=\mathrm{e}^{-w}$, so that $\varphi$ and $\psi$ belong to $\mathcal{M}_{\omega}$. We have

$$
u=\sum_{n \leqslant 0} \varphi_{n} S^{n-k} v, \quad S^{-k} v=\sum_{n \leqslant 0} \psi_{n} S^{n} u, \quad \text { and } \quad \bigvee_{n \leqslant 0} S^{n} u=\bigvee_{n \leqslant-k} S^{n} v
$$


In what follows we will need the notion of boundary value for functions $\varphi \in L_{\text {loc }}^{1}(\mathbb{D})$ such that $\bar{\partial} \varphi$, computed in the sense of distribution theory, is a measure of bounded variation on $\mathbb{D}$. This theory is developed in detail in [32]. Here we can restrict attention to the case where $\bar{\partial} \varphi \in L^{\infty}(\mathbb{D})$, so that $\mathcal{C}(\bar{\partial} \varphi)$ is continuous on $\mathbb{C}$ and $\varphi$ is continuous on $\mathbb{D}$, since $\varphi-\mathcal{C}^{+}(\bar{\partial} \varphi) \in \mathcal{H}(\mathbb{D})$. In this situation we define the "boundary value" of $\varphi$ on $\mathbb{T}$ to be the hyperfunction defined by the formula

$$
b(\varphi)=\left(\varphi-\mathcal{C}^{+}(\bar{\partial} \varphi), \mathcal{C}^{-}(\bar{\partial} \varphi)\right)
$$

We obtain

$$
\widehat{b(\varphi)}(n)=\frac{1}{\pi} \iint_{\mathbb{D}} \bar{\partial} \varphi(\xi) \xi^{-n-1} \mathrm{~d} m(\xi) \quad(n<0) .
$$

It follows from the Cauchy-Pompeiu formula that

$$
\frac{1}{\pi} \iint_{r \mathbb{D}} \bar{\partial} \varphi(\xi) \xi^{-n-1} \mathrm{~d} m(\xi)=\frac{1}{2 \mathrm{i} \pi} \int_{r \mathbb{T}} \varphi(\xi) \xi^{-n-1} \mathrm{~d} \xi
$$

for $n<0, r \in(0,1)$. Also

$$
\lim _{r \rightarrow 1^{-}} \int_{r \mathbb{T}} \mathcal{C}(\bar{\partial} \varphi)(\xi) \xi^{-n-1} \mathrm{~d} \xi=\int_{\mathbb{T}} \mathcal{C}(\bar{\partial} \varphi)(\xi) \xi^{-n-1} \mathrm{~d} \xi=0
$$

for $n \geqslant 0$, since $\mathcal{C}^{-}(\bar{\partial} \varphi) \in \mathcal{H}_{0}(\mathbb{C} \backslash \overline{\mathbb{D}})$, and we obtain

$$
\widehat{b(\varphi)}(n)=\lim _{r \rightarrow 1^{-}} \frac{1}{2 \mathrm{i} \pi} \int_{r \mathbb{T}} \varphi(\xi) \xi^{-n-1} \mathrm{~d} \xi \quad(n \in \mathbb{Z}) .
$$

Now assume that $\bar{\partial} \varphi \in L^{\infty}(\mathbb{D})$ and that

$$
\sup _{0 \leqslant r<1} \int_{0}^{2 \pi}\left|\varphi\left(r \mathrm{e}^{\mathrm{i} t}\right)\right|^{2} \mathrm{~d} t<+\infty
$$

Then $\varphi-\mathcal{C}^{+}(\partial \varphi) \in H^{2}, \lim _{r \rightarrow 1^{-}} \varphi\left(r \mathrm{e}^{\mathrm{i} t}\right)$ exists a.e. on $\mathbb{T}$ and it follows from (B.3) that $b(\varphi) \in L^{2}(\mathbb{T})$ and we have

$$
b(\varphi)\left(\mathrm{e}^{\mathrm{i} t}\right)=\lim _{r \rightarrow 1^{-}} \varphi\left(r \mathrm{e}^{\mathrm{i} t}\right) \quad \text { a.e. on } \mathbb{T} .
$$

Now let $\varphi$ be as above, and assume that $\psi \in L^{\infty}(\mathbb{D}), \bar{\partial} \psi \in L^{\infty}(\mathbb{D}), \varphi \bar{\partial} \psi \in L^{\infty}(\mathbb{D})$, so that $\bar{\partial}(\varphi \psi)=\varphi \bar{\partial} \psi+\psi \bar{\partial} \varphi \in L^{\infty}(\mathbb{D})$. We obtain

$$
b(\varphi \psi)=b(\varphi) b(\psi) .
$$

As in Section 2 we will denote by $L_{\sigma}$ the Legendre transform of $\sigma \in \mathcal{S}^{+}$. We will need the following technical result. 
LemMA B.2. - Let $\sigma \in \mathcal{S}^{+}$be eventually log-convex, and assume that the sequence $\left(\frac{\log \sigma^{-1}(n)}{\sqrt{n}}\right)_{n \geqslant 1}$ is eventually increasing. Let $\delta>1$ and $\varepsilon \in(0, \delta-1)$. Then there exists for every $f \in H_{\sigma^{*}}^{-}$a function $\varphi \in \mathcal{C}^{1}(\mathbb{D})$ satisfying the two following conditions:

(i) $|\varphi(\lambda)|=\mathrm{O}\left(L_{\sigma}^{-1-\varepsilon}\left(|\lambda|^{\delta}\right)\right)\left(|\lambda| \rightarrow 1^{-}\right)$;

(ii) $f=\mathcal{C}^{-}(\varphi)$.

Proof. - It follows from (4.10) that $(1-r) \log L_{\sigma}\left(r^{c}\right)$ is eventually increasing as $r \rightarrow 1^{-}$for every $c>0$. Fix $\delta>1, \varepsilon \in(0, \delta-1)$ and $\delta_{1} \in\left(1, \frac{\delta^{1 / 2}}{(1+\varepsilon)^{1 / 2}}\right)$. When $1-r$ is sufficiently small we have

$$
L_{\sigma}\left(r^{\delta}\right)^{1+\varepsilon} \leqslant L_{\sigma}\left(r^{\delta_{1}}\right)^{\frac{(1+\varepsilon)\left(1-r^{\delta_{1}}\right)}{1-r^{\delta}}}=L_{\sigma^{1 / \delta_{1}}}(r)^{\delta_{1} \frac{(1+\varepsilon)\left(1-r^{\delta_{1}}\right)}{1-r^{\delta}}}
$$

Hence there exist $r_{0} \in(0,1)$ and $\eta \in(0,1)$ satisfying

$$
L_{\sigma}\left(r^{\delta}\right)^{1+\varepsilon} \leqslant L_{\sigma^{1 / \delta_{1}}}^{1-\eta}(r) \quad\left(r_{0} \leqslant r<1\right)
$$

Now let $\alpha>3 / 2 \eta$, and set $\theta(n)=\sigma^{1 / \delta_{1}}(n) /(n+1)^{\alpha}$ for $n \geqslant 0$. Since

$$
\liminf _{n \rightarrow \infty} \frac{\log \sigma^{-1}(n)}{\sqrt{n}}>0
$$

we have $\theta(n) \geqslant \sigma(n)$ when $n$ is sufficiently large, and so $H_{\sigma^{*}}^{-} \subset H_{\theta^{*}}^{-}$. Let $f \in H_{\sigma^{*}}^{-}$. Since $1-\eta<1-\frac{3}{2 \alpha}$, it follows then from Proposition 2.5 that there exists a function $\rho \in w(\theta)$, which is continuously differentiable and strictly decreasing on $[0,1)$ and satisfies

$$
\left|\bar{\partial} \mathcal{D}_{\rho}\left(f^{-}\right)(\lambda)\right|=\mathrm{O}\left(L_{\theta}^{\eta-1}(|\lambda|) \quad\left(|\lambda| \rightarrow 1^{-}\right) .\right.
$$

But $\theta(n) \leqslant \sigma^{1 / \delta_{1}}(n)$ for $n \geqslant 0$, and so $L_{\sigma^{1 / \delta_{1}}}(r) \leqslant L_{\theta}(r)$ for $r \in[0,1)$. It follows then from (B.6) and Proposition 2.3 that the function $\varphi=\bar{\partial} \mathcal{D}_{\rho}(f)$ satisfies the required conditions.

The following factorization result is a quantitative version of [18, Theorem 6.3]. It is also related to Bourgain's theorem from [19].

THEOREM B.3. - Let $\omega \in \mathcal{S}$, and assume that $\omega$ satisfies the following conditions:

(1) $\omega(n)=1(n \geqslant 0)$;

(2) $\sum_{n<0} \frac{\log \omega(n)}{n^{2}}=+\infty$;

(3) $(\omega(-n))_{n \geqslant 1}$ is eventually log-concave;

(4) $\left(\frac{\log \omega(-n)}{\sqrt{n}}\right)_{n \geqslant 1}$ is eventually increasing.

Then for every $f \in L_{\omega}^{2}(\mathbb{T})$ and every $s<1 / 4$ there exists $g \in H^{2}, h \in H_{0}^{2}, k \geqslant 0$ satisfying

(i) $\limsup _{n \rightarrow \infty}|\hat{h}(n)| \omega^{s}(-n)<+\infty$;

(ii) $f\left(\mathrm{e}^{\mathrm{i} t}\right)=\mathrm{e}^{-\mathrm{i} k t} \mathrm{e}^{\bar{h}\left(\mathrm{e}^{\mathrm{i} t}\right)} g\left(\mathrm{e}^{\mathrm{i} t}\right)$ a.e. on $\mathbb{T}$.

Proof. - Set $\sigma(n)=\omega^{-1}(-n-1)$ for $n \geqslant 0$, so that $\sigma=\left(\omega^{*}\right)_{+}$, let $s>4, \delta \in(1, s / 4)$ and $\varepsilon \in(0, \delta-1)$. Let $f \in L_{\omega}^{2}(\mathbb{T}) \backslash\{0\}$, and set $f^{+}(\lambda)=\sum_{n=0}^{\infty} \hat{f}(n) \lambda^{n}$ for $|\lambda|<1$, $f^{-}(\lambda)=\sum_{n<0} \hat{f}(n) \lambda^{n}$ for $|\lambda|>1, F=f^{+}+\mathcal{C}^{+}(\varphi)$, where $\varphi \in \mathcal{C}^{1}(\mathbb{D})$ satisfies the conditions of Lemma B.2 with respect to $f, \delta$ and $\varepsilon$. We obtain

$$
|\bar{\partial} F(\lambda)|=\mathrm{O}\left(L_{\sigma}^{-1-\varepsilon}\left(|\lambda|^{\delta}\right)\right) \quad\left(|\lambda| \rightarrow 1^{-}\right) .
$$


Also $F \in \mathcal{C}^{1}(\mathbb{D})$ and, since $f^{+} \in H^{2}$, there exists $\psi \in H^{\infty}(\mathbb{D})$, with $\|\psi\|_{H^{\infty}(\mathbb{D})}=1$, such that $F \psi \in L^{\infty}(\mathbb{D})$. Set

$$
A=\left\{r \in[0,1)\left|\inf _{|\lambda|=r}\right| F(\lambda) \mid \geqslant L_{\sigma}^{-\varepsilon / 2}\left(r^{s}\right)\right\}
$$

and set $B=\bigcup_{r \in A} r \mathbb{T}$. For $k \geqslant 0$ set

$$
\begin{aligned}
& \Delta_{k}=\left\{\lambda \in \mathbb{C}\left|1-2^{-k} \leqslant\right| \lambda \mid \leqslant 1-2^{-k-1}\right\}, \\
& \Omega_{k}=\left\{\lambda \in \mathbb{C}\left|1-2^{-k}<\right| \lambda \mid<1\right\}, \\
& A_{k}=A \cap\left[1-2^{-k}, 1-2^{-k-1}\right]
\end{aligned}
$$

and $B_{k}=B \cap \Delta_{k}$. Set $\rho(r)=L_{\sigma}^{\varepsilon / 2}\left(r^{s}\right)$ for $r \in[0,1)$. It follows from (4.9), (4.10) and (B.7) that $F . \psi$ and $\rho$ satisfy the hypothesis of Lemma 4.1. Since $|F(\lambda)| \geqslant|F(\lambda)| \cdot|\psi(\lambda)|$ for $\lambda \in \mathbb{D}$, we deduce from Lemma 4.1(i) that there exists $k_{0} \geqslant 1$ for which we have

$$
\left|A_{k}\right| \geqslant 2^{-k-2} \quad\left(k \geqslant k_{0}\right)
$$

Now denote by $\chi_{k}$ the characteristic function of $A_{k}$ and set, for $\lambda \in \mathbb{C}$

$$
\begin{aligned}
& u_{k}(\lambda)=\frac{1}{\pi} \iint_{\Delta_{k}} \frac{\bar{\partial} F(\xi)}{\lambda-\xi} \mathrm{d} m(\xi) \quad(k \geqslant 0), \\
& v_{k}(\lambda)=\frac{1}{2\left|A_{k}\right|} \chi_{k}(|\lambda|) u_{k-1}(\lambda) \cdot \frac{\lambda}{|\lambda|} \quad(k \geqslant 1) .
\end{aligned}
$$

Since $u_{k-1}$ is analytic for $|\lambda|>1-2^{k}$, we have

$$
\frac{1}{2 \mathrm{i} \pi} \int_{r \mathbb{T}} \frac{u_{k-1}(\xi)}{\lambda-\xi} \mathrm{d} \xi=u_{k-1}(\lambda)
$$

for $r \in A_{k},|\lambda|>1-2^{-k-1}$. Since

$$
\frac{1}{\pi} \iint_{\mathbb{D}} \frac{v_{k}(\xi)}{\lambda-\xi}=\frac{1}{2 \pi\left|A_{k}\right|} \iint_{B_{k}} \frac{u_{k-1}(\xi)}{\lambda-\xi} \frac{\xi}{|\xi|} \mathrm{d} m(\xi),
$$

we obtain

$$
\frac{1}{\pi} \iint_{\mathbb{D}} \frac{v_{k}(\xi)}{\lambda-\xi} \mathrm{d} m(\xi)=u_{k-1}(\lambda) \quad\left(k \geqslant 1,|\lambda|>1-2^{-k-1}\right) .
$$

It follows from (B.7) that $\left\|u_{k-1}\right\|_{L^{\infty}(\mathbb{D})}=\mathrm{O}\left(L_{\sigma}^{-1-\varepsilon}\left[\left(1-2^{-k+1}\right)^{\delta}\right]\right)$. Also it follows from (4.9) and (4.10) that $L_{\sigma}(r) \mathrm{e}^{\frac{1}{r-1}} \rightarrow_{r \rightarrow \infty} \infty$, so that $2^{k}=\mathrm{o}\left(L^{\varepsilon / 2}\left[\left(1-2^{-k+1}\right)^{\delta}\right]\right.$. We obtain

$$
\left\|v_{k}\right\|_{L^{\infty}(\mathbb{D})}=\mathrm{o}\left(L_{\sigma}^{-1-\varepsilon / 2}\left[\left(1-2^{-k+1}\right)^{\delta}\right]\right) .
$$

Set $v(\lambda)=\sum_{k=1}^{\infty} v_{k}(\lambda)$. It follows from (B.10) that the series $\sum_{k=1}^{\infty} v_{k}$ converges in $L^{\infty}(\mathbb{D})$. Hence $\mathcal{C}(v)$ is bounded and continuous on $\mathbb{C}$, and it follows from (B.9) that we have

$$
\left.\mathcal{C}(v)\right|_{\mathbb{C} \backslash \overline{\mathbb{D}}}=\left.\mathcal{C}(\bar{\partial} F)\right|_{\mathbb{C} \backslash \overline{\mathbb{D}}} .
$$


When $1-|\lambda|$ is sufficiently small, we have $1-|\lambda|^{s / \delta} \geqslant 4(1-|\lambda|)$, so that $|\lambda|^{s} \leqslant\left(1-2^{-k+1}\right)^{\delta}$ for $\lambda \in \Delta_{k}$. It follows then from (B.10) that we have

$$
|v(\lambda)|=\mathrm{o}\left(L_{\sigma}^{-1-\varepsilon / 2}\left(|\lambda|^{s}\right)\right) \quad\left(|\lambda| \rightarrow 1^{-}\right)
$$

Now set $G=f^{+}+\mathcal{C}^{+}(v)$, so that $\bar{\partial} G=v$ and let $\lambda \in \Omega_{k+1}$, so that $u_{p-1}(\lambda)=\mathcal{C}^{+}\left(v_{p}\right)(\lambda)$ for $p \leqslant k$. We have

$$
\begin{aligned}
|F(\lambda)-G(\lambda)| & =\left|\mathcal{C}^{+}(\bar{\partial} F)(\lambda)-\mathcal{C}^{+}(v)(\lambda)\right| \\
& =\left|\sum_{p=k+1}^{\infty} u_{p-1}(\lambda)-\sum_{p=k+1}^{\infty} \mathcal{C}^{+}\left(v_{p}\right)(\lambda)\right| \\
& \leqslant \frac{1}{\pi} \iint_{\Omega_{k}} \frac{|\bar{\partial} F(\xi)|}{|\lambda-\xi|} \mathrm{d} m(\xi)+\frac{1}{\pi} \iint_{\Omega_{k+1}} \frac{|v(\xi)|}{|\lambda-\xi|} \mathrm{d} m(\xi) .
\end{aligned}
$$

By continuity, this inequality holds for $\lambda \in \Delta_{k+1}$. Using (B.7) and (B.10), we see that $\sup _{\lambda \in \Delta_{k+1}}|F(\lambda)-G(\lambda)|=\mathrm{o}\left(L_{\sigma}^{-1-\varepsilon / 2}\left[\left(1-2^{-k}\right)^{\delta}\right]\right)$ as $k \rightarrow \infty$. Since $|\lambda|^{s} \leqslant\left(1-2^{-k}\right)^{\delta}$ for $\lambda \in \Delta_{k+1}$ when $k$ is sufficiently large, we obtain

$$
|F(\lambda)-G(\lambda)|=\mathrm{o}\left(L_{\sigma}^{-1-\varepsilon / 2}\left(|\lambda|^{s}\right)\right) \quad\left(|\lambda| \rightarrow 1^{-}\right)
$$

It follows then from the definition of $B$ that we have

$$
\liminf _{\substack{|\lambda| \rightarrow 1^{-} \\ \lambda \in B}} L_{\sigma}^{\varepsilon / 2}\left(|\lambda|^{s}\right)|G(\lambda)| \geqslant 1 .
$$

Since $\bar{\partial} G=v, \bar{\partial} G$ being computed in the sense of distributions, $\bar{\partial} G(\lambda)=0$ for $\lambda \in \mathbb{D} \backslash B$. It follows from (B.12) and (B.14) that there exists $r_{1} \in(0,1)$ such that $G(\lambda) \neq 0$ and $|\bar{\partial} G(\lambda) / G(\lambda)| \leqslant L_{\sigma}^{-1}\left(|\lambda|^{s}\right)$ for $\lambda \in B, r_{1} \leqslant|\lambda|<1$. Set $U(\lambda)=0$ for $|\lambda|<r_{1}$ or for $\lambda \notin B$, $U(\lambda)=\bar{\partial} G(\lambda) / G(\lambda)$ for $\lambda \in B, r_{1} \leqslant|\lambda|<1$. We obtain

$$
|U(\lambda)| \leqslant L_{\sigma}^{-1}\left(|\lambda|^{s}\right) \quad(\lambda \in \mathbb{D}) .
$$

Now set $H=\mathcal{C}^{+}(U), h=\left.\mathcal{C}(U)\right|_{\mathbb{T}}, f_{1}=f \mathrm{e}^{-h}$. Since $H$ is bounded and continuous on $\mathbb{D}$, and since $\bar{\partial} \mathrm{e}^{-H}=U \mathrm{e}^{-H}, \bar{\partial} \mathrm{e}^{-H} \in L^{\infty}(\mathbb{D})$ and it follows from (B.15) that $G \bar{\partial} \mathrm{e}^{-H} \in L^{\infty}(\mathbb{D})$. It follows then from (B.5) that $f_{1}=b\left(G \mathrm{e}^{-H}\right)$, since

$$
\lim _{r \rightarrow 1^{-}} G\left(r \mathrm{e}^{\mathrm{i} t}\right)=\lim _{r \rightarrow 1^{-}} f^{+}\left(r \mathrm{e}^{\mathrm{i} t}\right)+\mathcal{C}(v)\left(\mathrm{e}^{\mathrm{i} t}\right)=f\left(\mathrm{e}^{\mathrm{i} t}\right) \quad \text { a.e. on } \mathbb{T} \text {. }
$$

Also $\bar{\partial}\left(G \mathrm{e}^{-H}\right)(\lambda)=0$ for $r_{1}<|\lambda|<1$, and so

$$
\hat{f}_{1}(n)=\frac{1}{\pi} \iint_{r_{1} \mathbb{D}} \bar{\partial}\left(G \mathrm{e}^{-H}\right)(\xi) \xi^{-n-1} \mathrm{~d} m(\xi)
$$

for $n<0$ and $\lim \sup _{n \rightarrow \infty}\left|\hat{f}_{1}(-n)\right|^{1 / n}<1$. Since $h \in \mathcal{C}(\mathbb{T}), f_{1} \in L^{2}(\mathbb{T})$.

Since $h=b(H), \hat{h}(n)=0$ for $n \geqslant 0$. Also it follows from (B.2) and (B.15) that we have, for $n \geqslant 0$,

$$
|\hat{h}(-n-1)| \leqslant \sup _{0 \leqslant r<1} L_{\sigma}^{-1}\left(r^{s}\right) r^{n} \leqslant \sup _{0 \leqslant r<1} L_{\sigma}^{-1 / s}\left(r^{s}\right) r^{n}=\sup _{0 \leqslant r<1}\left(L_{\sigma}^{-1}(r) r^{n}\right)^{1 / s} .
$$


Hence $\hat{h}(-n) \leqslant \sigma^{1 / s}(n)=\omega^{-1 / s}(-n-1)$ and $|\hat{h}(n)|=\mathrm{O}\left(\omega^{-1 / s}(n)\right)$ as $n \rightarrow-\infty$. Since $f=\mathrm{e}^{h} f_{1}$, the theorem follows then immediately from Proposition B.1.

In order to obtain our second factorization theorem we will need the following extension of formula (B.5).

Lemma B.4. - Let $\varphi \in L_{\text {loc }}^{1}(\mathbb{D}), \psi \in L^{\infty}(\mathbb{D})$, with $\bar{\partial} \varphi \in L^{\infty}(\mathbb{D})$. Assume that there exists a continuous non-negative non-increasing function $\rho$ on $[0,1)$ such that $\operatorname{ess} \sup |\varphi(\lambda)| \rho(|\lambda|)<$ $+\infty$ and $|\bar{\partial} \psi(\lambda)| \leqslant \rho(|\lambda|)$ a.e. on $\mathbb{D}$.

Then

$$
b(\widehat{\varphi \psi})(n)=\lim _{r \rightarrow 1^{-}} \sum_{p \in \mathbb{Z}} r^{|p|} \widehat{b(\varphi)}(p) \widehat{b(\psi)}(n-p) \quad(n \in \mathbb{Z}) .
$$

Proof. - Set $\varphi^{-}=\mathcal{C}^{+}(\varphi)$, so that $b \widehat{\left(\varphi^{-}\right)}(n)=0$ for $n \geqslant 0$ and $b \widehat{\left(\varphi^{-}\right)}(n)=b \widehat{(\varphi)}(n)$ for $n<0$. Then

$$
\sum_{p<0}|\widehat{b(\varphi)}(p)| \cdot|\widehat{b(\psi)}(n-p)|=\sum_{p<0}\left|b\left(\widehat{\left.\varphi^{-}\right)(p)}|.| b \widehat{(\psi}\right)(n-p)\right|<+\infty
$$

and $\left.\widehat{b\left(\varphi^{-}\right.} \psi\right)(n)=\sum_{p<0} b \widehat{(\varphi)}(p) b \widehat{(\psi)}(n-p)$, by (B.5). Hence we can restrict attention to the case where $\varphi \in \mathcal{H}(\mathbb{D})$.

Let $s \in[0,1)$ and $r \in(s, 1)$. Then $b(\psi) \in L^{\infty}(\mathbb{T})$ and we have, for $n \in \mathbb{Z}$

$$
\sum_{p=0}^{\infty} r^{p} \widehat{b(\varphi)}(p) \widehat{b(\psi)}(n-p)=\frac{r^{n}}{2 \mathrm{i} \pi} \int_{r \mathbb{T}} \varphi(\xi) b(\psi)\left(\frac{\xi}{r}\right) \xi^{-n-1} \mathrm{~d} \xi
$$

We obtain, by the Cauchy-Pompeiu formula

$$
\begin{aligned}
& \left|\frac{1}{2 \mathrm{i} \pi} \int_{r \mathbb{T}} \varphi(\xi) \psi(\xi) \xi^{-n-1} \mathrm{~d} \xi-\sum_{p=0}^{\infty} r^{p} \widehat{b(\varphi)(p) b(\psi)}(n-p)\right| \\
& \leqslant \frac{1}{2 \pi}\left|\int_{s \mathbb{T}}\left[\varphi(\xi) \psi(\xi) \xi^{-n-1}-r^{n} \varphi(\xi) \psi\left(\frac{\xi}{r}\right)\right] \mathrm{d} \xi\right| \\
& \quad+\frac{1}{\pi} \int_{s<|\xi|<r}\left[|\varphi(\xi)||\bar{\partial} \psi(\xi)|+r^{n-1}|\varphi(\xi)|\left|\bar{\partial} \psi\left(\frac{\xi}{r}\right)\right|\right]\left|\xi^{-n-1}\right| \mathrm{d} m(\xi) .
\end{aligned}
$$

There exists $M>0$ such that $|\varphi(\xi)| \cdot|\bar{\partial} \psi(\xi)| \leqslant M$ and $|\varphi(\xi)||\bar{\partial} \psi(\xi / r)| \leqslant M$ a.e. for $|\xi| \leqslant r, r \in[0,1)$. Hence

$\left.\limsup _{r \rightarrow 1^{-}} \mid \frac{1}{2 \mathrm{i} \pi} \int_{r \mathbb{T}} \varphi(\xi) \psi(\xi) \xi^{-n-1} \mathrm{~d} \xi-\sum_{p=0}^{\infty} r^{p} \widehat{b(\varphi)}(p) b \widehat{(\psi}\right)(n-p) \mid \leqslant M\left(1+|s|^{-|n|-1}\right)\left(1-s^{2}\right)$

for every $s \in(0,1)$, and the lemma follows from (B.3).

For $\omega \in \mathcal{S}, s>0$ set as in Section $5 \omega_{(s)}(n)=\omega(n)(n \geqslant 0), \omega_{(s)}(n)=\omega^{s}(n)(n \leqslant 0)$. The following factorization result, which is slightly more precise than Corollary 5.3 , is a quantitative version of [14, Theorem 4] and [18, Theorem 6.1].

THEOREM B.5. - Let $\omega \in \mathcal{S}$, and assume that $\omega$ satisfies the following conditions: 
(1) $\sum_{n<0} \frac{\log \omega(n)}{n^{2}}=+\infty$;

(2) $(\omega(-n))_{n \geqslant 1}$ is eventually log-concave;

(3) $\left(\frac{\log \omega(-n)}{n}(\log n)^{A}\right)_{n \geqslant 1}$ is eventually increasing for some $A>0$;

(4) $\limsup _{n \rightarrow \infty} \frac{\log \bar{\omega}_{+}(n)}{\log \omega(-n)}<1 / 4$;

(5) $\lim \sup _{n \rightarrow \infty} \frac{\log ^{+} \omega^{-1}(n)}{\log \omega(-n)}<1 / 64$.

Then for every $u \in \ell_{\omega}$ and every $s<1 / 4$ there exists $v \in \ell_{\omega}^{+}, k \geqslant 0$ and $w=\left(w_{n}\right)_{n<0}$ satisfying the following conditions:

(i) $\sum_{n<0}\left|w_{n}\right| \tilde{\omega}_{(s)}(n)<+\infty$;

(ii) $S^{k} u=\mathrm{e}^{w} * v$.

Proof. - Set again $\sigma=\left(\omega^{*}\right)_{+}$and set

$$
\beta=\limsup _{n \rightarrow \infty} \frac{\log \bar{\omega}_{+}(n)}{\log \omega(-n)}, \quad \gamma=\limsup _{n \rightarrow \infty} \frac{\log ^{+} \omega^{-1}(n)}{\log \omega(-n)}, \quad K=\min \left(\beta^{-1}, 2^{-4} \gamma^{-1}\right),
$$

so that $K>4$. Let $s>4$, and let $t \in(4, \min (s, K)), \delta \in(1, t / 4)$ and $\varepsilon \in(0, \delta-1)$. Let $u \in \ell_{\omega} \backslash\{0\}$ and set $F=u^{+}+\mathcal{C}^{+}(\varphi)$, where $\varphi \in \mathcal{C}^{1}(\mathbb{D})$ satisfies the condition of Lemma B.2 with respect to $u^{-}, \delta$ and $\varepsilon$. Also set $\rho(r)=L_{\sigma}^{\varepsilon / 2}\left(r^{t}\right)$ for $r \in[0,1)$. Then $b(\widehat{F})=u$, and $F$ satisfies (B.7).

For $\alpha \in(0,1), c<\gamma^{-1}$ there exists $M>0$ such that we have, for $\lambda \in \mathbb{D}$

$$
\left|u^{+}(\lambda)\right| \leqslant M\left(\sum_{n=0}^{\infty} \sigma^{-1 / c}(n) \cdot|\lambda|^{n}\right) \leqslant M\left(1-|\lambda|^{\alpha}\right) \log L_{\sigma^{1 / c}}\left(|\lambda|^{1-\alpha}\right) .
$$

Hence

$$
\log ^{+}\left|u^{+}(\lambda)\right|=\mathrm{o}\left(\log L_{\sigma^{1 / c}}\left(|\lambda|^{1-\alpha}\right)\right)=\mathrm{o}\left(\rho\left(|\lambda|^{\frac{c(1-\alpha)}{t}}\right)\right)
$$

as $|\lambda| \rightarrow 1^{-}$.

Since $\gamma^{-1}>16 t$, we can choose $c$ and $\alpha$ such that $\frac{c(1-\alpha)}{t}>16$. Let $\eta \in\left(16, \frac{c(1-\alpha)}{t}\right)$. It follows from Proposition 5.1 that we have

$$
|F(\lambda)| \rho\left(|\lambda|^{\eta}\right)^{|\lambda|-1} \underset{|\lambda| \rightarrow 1^{-}}{\rightarrow} 0 .
$$

Since the sequence $\left(\frac{\log \omega^{d}(-n)}{n^{\alpha}}\right)_{n \geqslant 1}$ is eventually increasing for every $\alpha \in(0,1)$ and every $d>0$ it follows again from [43] that $(1-r)^{k} \log \rho(r)$ is eventually increasing as $r \rightarrow 1^{-}$for every $k>0$. Since $F$ satisfies (B.7) and (B.16), and since $b(F)=u \neq 0$, it follows then from Lemma 5.2 that there exists $k_{0} \geqslant 1$ such that $\left|A_{k}\right| \geqslant 2^{-k-2}$ for $k \geqslant k_{0}$, where

$$
A_{k}=\left\{r \in\left[1-2^{-k}, 1-2^{-k-1}\right]\left|\inf _{|\lambda|=r}\right| F(\lambda) \mid \geqslant \rho(r)\right\}
$$

Using the same construction as in the proof of Theorem B.3 we obtain two functions $G \in L_{\text {loc }}^{1}(\mathbb{D})$ and $U \in L^{\infty}(\mathbb{D})$, with $\bar{\partial} G \in L^{\infty}(\mathbb{D})$, and $r_{1} \in(0,1)$ such that $(\bar{\partial} G)(\lambda)-G(\lambda) U(\lambda)=0$ for $r_{1}<|\lambda|<1,|U(\lambda)| \leqslant L_{\sigma}^{-1}\left(|\lambda|^{t}\right)$ for $\lambda \in \mathbb{D}$ and $|F(\lambda)-G(\lambda)|=\mathrm{O}\left(L_{\sigma}^{-1-\varepsilon / 2}\left(|\lambda|^{t}\right)\right)$ as $|\lambda| \rightarrow 1^{-}$. Set again $H=\mathcal{C}^{+}(U), h=\left.\mathcal{C}(U)\right|_{\mathbb{T}}$, so that $\hat{h}(n)=0$ for $n \geqslant 0$. Set $w=(\hat{h}(n))_{n<0}$, $v=b\left(\widehat{\mathrm{e}^{-H} G}\right)$. We have again $b(G)=b(F)=u$ and $|\hat{h}(n)|=\mathrm{O}\left(\omega^{-1 / t}(n)\right)$ as $n \rightarrow-\infty$. It 
follows from Proposition 5.1 that

$$
\limsup _{n \rightarrow \infty} \frac{\log \tilde{\omega}_{(1 / s)}(n)}{\log \omega^{1 / t}(-n)}<1 .
$$

Since $\sum_{n<0} \omega^{-\alpha}(n)<+\infty$ for every $\alpha>0$, this shows that $\sum_{n<0}|\hat{h}(n)| \tilde{\omega}_{(1 / s)}(n)<+\infty$, and $w$ satisfies (i). In particular $w$ is a convolution multiplier on $\ell_{\omega_{(1 / s)}}$. Hence $\mathrm{e}^{w}=\sum_{n=0}^{\infty} \frac{w^{* n}}{n !}$ is also a convolution multiplier on $\ell_{\omega_{(1 / s)}}$. Since $\sum_{n<0}|\hat{h}(n)|<+\infty$, we have $\mathrm{e}^{w}=\widehat{\mathrm{e}^{h}}=\widehat{b\left(\mathrm{e}^{H}\right)}$ and $\mathrm{e}^{-w}=\widehat{\mathrm{e}^{-h}}=\widehat{b\left(\mathrm{e}^{-H}\right)}$. Also, since $\ell_{\tilde{\omega}_{(1 / s)}}^{1}(\mathbb{Z})$ is a Banach algebra with respect to convolution, we have $\sum_{n \in \mathbb{Z}}\left|\widehat{b\left(\mathrm{e}^{-H}\right)}(p)\right|\left|u_{n-p}\right|<+\infty$ for $n \in \mathbb{Z}$.

Since $|F(\lambda)-G(\lambda)| \rightarrow|\lambda| \rightarrow 1^{-} 0$ it follows from (B.16) that $|G(\lambda)|=\mathrm{O}\left(L_{\sigma}^{\varepsilon_{2}(1-|\lambda|)}\left(|\lambda|^{16 t}\right)\right)$ as $|\lambda| \rightarrow 1^{-}$. Also

$$
\left|\left(\bar{\partial} \mathrm{e}^{-H}\right)(\lambda)\right|=\mathrm{O}(|U(\lambda)|)=\mathrm{O}\left(L_{\sigma}^{-1}\left(|\lambda|^{t}\right)\right) \quad \text { as }|\lambda| \rightarrow 1^{-} .
$$

It follows then from Lemma B.4 that

$$
b\left(\widehat{\mathrm{e}^{-H}} G\right)(n)=\lim _{r \rightarrow 1^{-}} \sum_{p \in \mathbb{Z}} r^{|p|} b\left(\widehat{\mathrm{e}^{-H}}\right)(p) b(\widehat{G})(n-p)=\sum_{p=0}^{\infty} b\left(\widehat{\mathrm{e}^{-H}}\right)(p) u_{n-p}
$$

for $n \in \mathbb{Z}$.

Hence $v=b\left(\widehat{\mathrm{e}^{-H}} G\right)=\mathrm{e}^{-w} * u \in \ell_{\omega}$. Since $\bar{\partial}\left(\mathrm{e}^{-H} G\right)=(\bar{\partial} G-G U) \mathrm{e}^{-H}$ vanishes for $r_{1}<|\lambda|<1$, we see as in the proof of Theorem B.3 that $\limsup _{n \rightarrow-\infty}\left|v_{n}\right|^{1 /|n|}<1$, and the theorem follows then immediately from Proposition B.1.

\section{REFERENCES}

[1] Abkar A., Hedenmalm H., Invariant subspaces on multiply connected domains, Publ. Math. 42 (1998) 521-557.

[2] Aleman A., Richter S., Ross W.T., Pseudocontinuations and the Backward Shift, Indiana Math. J. 47 (1) (1998) 223-276.

[3] Aleman A., Richter S., Sundberg C., Beurling's theorem for the Bergman space, Acta Math 177 (1996) 275-310.

[4] Apostol C., Hyperinvariant subspaces for bilateral weighted shifts, J. Int. Eq. Op. Theory 7 (1984) $1-9$.

[5] Atzmon A., An operator without invariant subspace on a nuclear Fréchet space, Ann. of Math. 117 (1983) 660-694.

[6] Atzmon A., An operator on a Fréchet space with no common invariant subspace with its inverse, J. Funct. Anal. 55 (1984) 68-77.

[7] AtZMOn A., Nuclear Fréchet spaces of entire functions with transitive differentiation, J. Analyse Math. 60 (1993) 1-19.

[8] Atzmon A., Entire functions, invariant subspaces and Fourier transforms, Israel Math. Conference Proceedings 11 (1997) 37-52.

[9] Atzmon A., Weighted $L^{p}$ spaces of entire functions, Fourier transforms and invariant subspaces, Preprint.

[10] ATZMOn A., The existence of self-adjoint translation invariant subspaces on symmetric self-adjoint sequence spaces on $\mathbb{Z}$, J. Funct. Anal. 178 (2000) 372-380.

[11] Atzmon A., Sodin M., Completely indecomposable operators and a uniqueness theorem of Cartwright-Levinson type, J. Funct. Anal. 169 (1999) 164-188. 
[12] Beurling A., Mittag-Leffler lectures in Complex Analysis (1977-78); Collected works of A. Beurling, 361-443.

[13] Beurling A., Malliavin P., On Fourier transforms of measures with compact support, Acta Math. 107 (1962) 291-309.

[14] BORICHEV A., Boundary uniqueness theorems for almost analytic functions, and asymmetric algebras of sequences, Math. USSR Sb. 64 (2) (1989) 323-338.

[15] BORICHEV A., Invariant subspaces of given index in Banach spaces of analytic functions, J. Reine Angew. Math. 505 (1998) 23-44.

[16] Borichev A., Hedenmalm H., Completeness of translates in weighted spaces on the half-line, Acta Math. 174 (1995) 1-84.

[17] Borichev A., Hedenmalm H., Volberg A., Large Bergman spaces: invertibility, cyclicity, and subspaces of arbitrary index, Preprint.

[18] Borichev A., Volberg A., Uniqueness theorems for almost analytic functions, Leningrad Math. J. 1 (1990) 157-190.

[19] Bourgain J., A problem of Douglas and Rudin of factorization, Pacific J. Math. 121 (1) (1986) 47-50.

[20] Brown S., Chevreau B., Pearcy C., On the structure of contraction operators II, J. Funct. Anal. 76 (1988) 269-293.

[21] Domar Y., Translation invariant subspaces of weighted $\ell^{p}$ and $L^{p}$ spaces, Math. Scand. 49 (1981) 133-144.

[22] Domar Y., Entire functions of order $\leqslant 1$, with bounds on both axes, Ann. Acad. Sci. Fenn. 22 (1997) 339-348.

[23] Dynkin E.M., Functions with a given estimate for $\partial f / \partial \bar{z}$ and $N$. Levinson's theorem, Math. Sb. 81 (1972) 182-190.

[24] El Fallah O., Kellay K., Sous-espaces biinvariants pour certains shifts à poids, Ann. Inst. Fourier 48 (1998) 1543-1588.

[25] Esterle J., Singular inner functions and biinvariants subspaces for dissymmetric weighted shifts, J. Funct. Anal. 44 (1997) 64-104.

[26] Esterle J., Countable inductive limits of Fréchet algebras, J. Ann. Math. 71 (1997) 195-204.

[27] Esterle J., Exact factorization never holds for Banach spaces of sequences, St. Petersburg Math. J. 12 (2001) 869-874.

[28] Esterle J., Apostol's bilateral weighted shifts are hyperreflexive, Operator Theory: Advances and Applications 127 (2001) 243-266.

[29] Esterle J., GAy R., Product of hyperfunctions on the circle, Israel J. Math. 116 (2000) 271-283.

[30] Esterle J., Volberg A., Sous-espaces invariants par translation de certains espaces de Hilbert de suites quasi-analytiquement pondérées, C. R. Acad. Sci. Paris, Ser. A 326 (1998) 295-300.

[31] Esterle J., Volberg A., Analytic left-invariant subspaces of weighted Hilbert spaces of sequences, J. Op. Theory 45 (2001) 265-301.

[32] HARlouchet I., Trace de Cauchy pour certaines fonctions localement intégrables sur un ouvert borné de $\mathbb{C}$, submitted.

[33] HaRlouchet I., Idéaux fermés de certaines algèbres de Beurling quasi-analytiques sur le cercle unité, J. Math. Pures Appl. 79 (2000) 863-899.

[34] Hedenmalm H., A comparison between the closed modular ideals of $\ell^{1}(\omega)$ and $L^{1}(\omega)$, Math. Scand. 58 (1986) 275-300.

[35] Hedenmalm H., Bounded analytic functions and closed ideals, J. Anal. Math. 48 (1987) 142-166.

[36] Hedenmalm H., Korenblum B., Zhu K., The Theory of Bergman Spaces, GTM 199, SpringerVerlag, 2000.

[37] Hedenmalm H., Volberg A., Zero-free invariant subspaces in weighted Bergman spaces with critical topology, Preprint.

[38] Helson H., Lectures on Invariant Subspaces, Academic Press, 1954.

[39] Hitт D., Invariant subspaces of $H^{2}$ of an annulus, Pacific J. Math. 134 (1) (1988) 101-120.

[40] Kellay K., Fonctions intérieures et vecteurs bicycliques, Archiv. der Math. 77 (2001) 253-264.

[41] Korenblum B., Outer functions and cyclic elements in Bergman spaces, J. Funct. Anal. 115 (1993) $104-118$. 
[42] Matsaev V.I., Mogulskit E.Z., A division theorem for analytic functions with a given majorant and some of its applications, J. Soviet Math. 14 (1980) 1078-1091.

[43] NiKOLSKII N., Selected problems in weighted approximations and spectral analysis, Proc. Steklov Inst. Math. 120 (1974).

[44] NikOLSKII N., Treatise on the Shift Operator, Springer-Verlag, Berlin, 1986.

[45] NikOLSKII N., Yngve Domar's forty years in harmonic analysis, Festschrift in honour of Lennart Carleson and Yngve Domar (Uppsala, 1993), Acta Univ. Upsaliensis 58 (1995) 45-78.

[46] SARAson D., The $H^{p}$-spaces of an annulus, Mem. Amer. Math. Soc. 56 (1965).

[47] SARASON D., Nearly invariant subspaces for the backward shift, Operator Theory Adv. Appl. 35 (1988) 481-493.

[48] SHIElDS A., Weighted shift operators and analytic function theory, in: Pearcy C. (Ed.), Topics in Operator Theory, Amer. Math. Soc. Math. Surveys, Vol. 13, 1974, pp. 49-128.

[49] Volberg A., The logarithm of an almost analytic function is summable, Soviet Math. Dokl. 26 (1982) 238-243.

[50] Volberg A., Asymptotically holomorphic functions and certain of their applications, Proc. I.C.M. Kyoto II (1990) 959-967.

[51] Volberg A., Joricke B., Summability of the logarithm of an almost analytic function and a generalization of the Levinson-Cartwright theorem, Math. USSR Sb. 58 (1987) 337-349.

[52] WIENER N., Tauberian theorems, Ann. of Math. 33 (1932) 1-100.

[53] YAKUBOvitch D., Invariant subspaces of the operator of multiplication by $z$ in the space $E_{p}$ in a multiply connected domain, LOMI, Stek. Akad. Nauk. SSSR 178 (1989) 166-183.

(Manuscript received June 17, 2000; accepted, after revision, October 6, 2000.)

\author{
Jean ESTERLE \\ Laboratoire de Mathématiques Pures, \\ UPRESA 5467, \\ Université de Bordeaux I, \\ 351, cours de la Libération, \\ 33405 Talence cedex, France \\ E-mail: esterle@math.u-bordeaux.fr
}

Alexander VolBERG

Department of Mathematics,

Michigan State University,

East Lansing, MI 48824, USA

E-mail: volberg@math.msu.edu 